\title{
Linear Stability and instability of relativistic Vlasov-Maxwell systems
}

\author{
Zhiwu Lin \\ Courant Institute \\ Walter Strauss \\ Brown University
}

\begin{abstract}
We consider the linear stability problem for a symmetric equilibrium of the relativistic Vlasov-Maxwell (RVM) system. For an equilibrium whose distribution function depends monotonically on the particle energy, we obtain a sharp linear stability criterion. The growing mode is proved to be purely growing and we get a sharp estimate of the maximal growth rate. In this paper we specifically treat the periodic $1 \frac{1}{2} \mathrm{D}$ case and the $3 \mathrm{D}$ whole-space case with cylindrical symmetry. We explicitly illustrate, using the linear stability criterion in the $1 \frac{1}{2} \mathrm{D}$ case, several stable and unstable examples.
\end{abstract}

(c) 2000 Wiley Periodicals, Inc.

\section{Contents}

1. Introduction 1

2. Linear stability of periodic $1 \frac{1}{2}$ dimensional RVM 9

3. Linear Stability of Axisymmetric 3D RVM 27

4. Examples in the $1 \frac{1}{2} \mathrm{D}$ case 42

Appendix $\quad 55$

$\begin{array}{ll}\text { Bibliography } & 60\end{array}$

\section{Introduction}

We consider a plasma at high temperature, of low density such that collisions can be ignored compared with the electromagnetic forces. Such a collisionless plasma is modeled by the relativistic Vlasov-Maxwell (RVM) system. We assume all physical constants like the speed of light and the mass of particles to be 1 , for the sole purpose of simplifying our notation. All the results we obtained below can be modified straightforwardly to apply to the true physical situations with masses, charges, etc. 
Let $f^{ \pm}(t, x, v)$ be the ion and electron distribution functions, $\mathbf{E}(t, x)$ and $\mathbf{B}(t, x)$ be the electric and magnetic fields and $\mathbf{E}^{\text {ext }}, \mathbf{B}^{\text {ext }}$ be the external fields. Then we have the RVM system

$$
\partial_{t} f^{ \pm}+\hat{v} \cdot \nabla_{x} f^{ \pm} \pm\left(\mathbf{E}+\mathbf{E}^{e x t}+\hat{v} \times\left(\mathbf{B}+\mathbf{B}^{e x t}\right)\right) \cdot \nabla_{v} f^{ \pm}=0,
$$

$$
\partial_{t} \mathbf{E}=\nabla \times \mathbf{B}-j, \nabla \cdot \mathbf{E}=\rho, \rho=\int\left(f^{+}-f^{-}\right) d v,
$$

$$
\partial_{t} \mathbf{B}=-\nabla \times \mathbf{E}, \nabla \cdot \mathbf{B}=0, \mathbf{j}=\int \hat{v}\left(f^{+}-f^{-}\right) d v,
$$

where $\hat{v}=v /\langle v\rangle$ and $\langle v\rangle=\sqrt{1+|v|^{2}}$. In many physical problems ([3], [4]), a nonneutral plasma is also considered, where there is only a single species of particle. One of the central problems in the theory of plasmas is to understand plasma stability and instability ([27], [30]). Most stability studies ([6], [7]) are based on macroscopic MHD models. However, many plasma instability phenomena have an essentially microscopic nature, for which kinetic models like Vlasov-Maxwell are required. Moreover, the collision-dominant assumption required in deriving MHD models is often not justified in physical situations, such as in nuclear fusion ([6]) and in astrophysics ([28]). The Vlasov-Maxwell system is a rather accurate description of a plasma when collisions are negligible, as occurs in many physical situations. So we expect that an understanding of stability of Vlasov plasmas could also provide the necessary theoretical ground to compare and test stability results from various approximating models like MHD.

The stability problem of Vlasov plasmas is very complicated partly because of its collective nature. More precisely, the instability in Vlasov plasmas is usually due to the collective behavior of all the particles. This makes the instability problem highly nonlocal and difficult to study both analytically and numerically. In the physics literature (e.g. [30]), usually only the stability of a homogeneous equilibrium with vanishing electromagnetic fields is treated. In this case, one can get a dispersion relation which is often an explicit algebraic equation and is rather easy to study analytically. A beautiful classical result of this type is Penrose's sharp linear instability criterion (see [27]) for a homogeneous equilibrium of the Vlasov-Poisson system. However, even for a homogeneous equilibrium the stability problem becomes quite complicated when magnetic effects are included, as for the Bernstein modes in a constant magnetic field ([30]).

The stability problem for inhomogeneous (spatially-dependent) equilibria with nonzero electromagnetic fields is much more complicated and so far there are few results. In [11], Guo and Strauss developed a sophisticated perturbation argument to obtain the instability of weakly inhomogeneous BGK waves of the VlasovPoisson system that are close to Penrose's unstable homogeneous equilibrium. This idea was further developed in their subsequent papers ([14], [12], [13]). In [20], Z. Lin proved that an arbitrary periodic BGK wave is unstable under perturbations 
of double the period, using a new method of dispersion operators together with a continuation argument.

In the present paper we study the linear stability of a symmetric equilibrium of RVM under perturbations with certain symmetries. We consider two RVM models, the $1 \frac{1}{2} \mathrm{D}$ periodic case with $x \in \mathbb{R}, v \in \mathbb{R}^{2}$ and the $3 \mathrm{D}$ case in the whole space with cylindrical symmetry. However, our methods are certainly applicable to other RVM models and will yield similar results. In a subsequent paper [25], we study the nonlinear stability and instability problem for $1 \frac{1}{2} \mathrm{D}$ RVM.

THE 3D CASE. Now we state our main result for the cylindrical 3D case. As mentioned in the appendix, there exists no plasma equilibrium of the 3D RVM model in the whole space without external fields. So it is necessary to include an external field. To simplify notation we consider a 3D nonneutral electron plasma with an external field, which indeed occurs in many important physical situations ([3], [4]). Instead of $f^{-}$, we use the notation $f$ in (1.1) for the electrons. The equilibrium we consider is cylindrically symmetric with electron distribution $f^{0}=$ $\mu(e, p)$, where

$$
\begin{gathered}
e=\sqrt{1+|v|^{2}}-\phi^{0}(r, z)-\phi^{e x t}(r, z), \\
p=r\left(v_{\theta}-A_{\theta}^{0}(r, z)-A_{\theta}^{e x t}(r, z)\right)
\end{gathered}
$$

and with equilibrium fields

$$
\mathbf{E}^{0}=-\partial_{r} \phi^{0} \vec{e}_{r}-\partial_{z} \phi^{0} \vec{e}_{z}, \mathbf{B}^{0}=-\partial_{z} A_{\theta}^{0} \vec{e}_{r}+\frac{1}{r} \partial_{r}\left(r A_{\theta}^{0}\right) \vec{e}_{z} .
$$

To be an equilibrium, $\left(A_{\theta}^{0}, \phi^{0}\right)$ must satisfy the elliptic system

$$
\begin{gathered}
\Delta \phi^{0}=\partial_{z z} \phi^{0}+\partial_{r r} \phi^{0}+\frac{1}{r} \partial_{r} \phi^{0}=\int \mu d v \\
\left(\Delta-\frac{1}{r^{2}}\right) A_{\theta}^{0}=\partial_{z z} A_{\theta}^{0}+\partial_{r r} A_{\theta}^{0}+\frac{1}{r} \partial_{r} A_{\theta}^{0}-\frac{1}{r^{2}} A_{\theta}^{0}=\int \hat{v}_{\theta} \mu d \nu .
\end{gathered}
$$

The derivation of this system from (1.1) can be found in [3] or [1]. Here we use cylindrical coordinates $(r, \theta, z)$ and denote by $\left(\vec{e}_{r}, \vec{e}_{\theta}, \vec{e}_{z}\right)$ the standard basis. We also assume axisymmetry of the external fields in the form

$$
\begin{aligned}
& \mathbf{E}^{\text {ext }}=-\partial_{r} \phi^{e x t}(r, z) \vec{e}_{r}-\partial_{z} \phi^{e x t} \vec{e}_{z}, \\
& \mathbf{B}^{\text {ext }}=-\partial_{z} A_{\theta}^{\text {ext }}(r, z) \vec{e}_{r}+\frac{1}{r} \partial_{r}\left(r A_{\theta}^{e x t}\right) \vec{e}_{z} .
\end{aligned}
$$

We assume a confined plasma equilibrium with compact support $S$ for $f^{0}$. Compact support is a realistic assumption for a confined plasma. We make the further assumption that $f^{0}$ and $\mathbf{E}^{0}, \mathbf{B}^{0}$ are continuous everywhere, including on the boundary of the support. In the appendix we show that with properly chosen external fields, an example of a continuous nonneutral plasma equilibrium with support in a torus is constructed. For such an equilibrium, the internal modes have distribution 
functions supported in $S$. Another important assumption is that $\partial \mu / \partial e=\mu_{e}<0$ inside $S$. This condition is widely believed to make the equilibrium more likely to be stable ([3], [29]). We consider the stability of such an equilibrium under perturbations preserving cylindrical symmetry.

In order to state our theorem, we define certain operators acting on the cylindrically symmetric functions $h \in L^{2}\left(\mathbb{R}^{3}\right)$ by

$$
\begin{gathered}
\mathscr{A}_{1}^{0} h=-\partial_{z z} h-\partial_{r r} h-\frac{1}{r} \partial_{r} h-\int \mu_{e} d v h+\int \mu_{e} \mathscr{P}(h) d v \\
\mathscr{A}_{2}^{0} h=-\partial_{z z} h-\partial_{r r} h-\frac{1}{r} \partial_{r} h+\frac{1}{r^{2}} h-\int \hat{v}_{\theta} \mu_{p} d v r h-\int \hat{v}_{\theta} \mu_{e} \mathscr{P}\left(\hat{v}_{\theta} h\right) d v \\
\mathscr{B}^{0} h=\int \mu_{e} \mathscr{P}\left(\hat{v}_{\theta} h\right) d v-\int \hat{v}_{\theta} \mu_{e} d v h
\end{gathered}
$$

and

$$
\mathscr{L}^{0}=\left(\mathscr{B}^{0}\right)^{*}\left(\mathscr{A}_{1}^{0}\right)^{-1} \mathscr{B}^{0}+\mathscr{A}_{2}^{0} .
$$

where $\mathscr{P}$ is the projection operator of $L_{\left|\mu_{e}\right|}^{2}$ onto $\operatorname{ker} D$. Here $D$ denotes the transport operator associated with the steady fields, namely

$$
D=\hat{v} \cdot \nabla_{x}+\left(\mathbf{E}^{0}+\mathbf{E}^{e x t}+\hat{v} \times\left(\mathbf{B}^{0}+\mathbf{B}^{\text {ext }}\right)\right) \cdot \nabla_{v}
$$

and $L_{\left|\mu_{e}\right|}^{2}$ is the $\left|\mu_{e}\right|$-weighted $L_{x, v}^{2}$ space. We will prove in Lemma 3.1 that these operators are well-defined and that $\mathscr{L}^{0}$ is self-adjoint. Our main result is

Theorem 1.1. Consider a nonnegative axisymmetric equilibrium $\left(f^{0}=\mu(e, p), \mathbf{E}^{0}, \mathbf{B}^{0}\right)$ as defined above. Assume $\mu_{e}<0$ inside $S$. For axisymmetric perturbations, we have following results.

(i) $\mathscr{L}^{0} \geq 0$ implies spectral stability. That is, if $\mathscr{L}^{0} \geq 0$ then there does not exist a growing mode.

(ii) Any growing mode must be purely growing, that is, if

$$
\left[e^{\lambda t} f(x, v), e^{\lambda t} \mathbf{E}(x), e^{\lambda t} \mathbf{B}(x)\right] \quad(\operatorname{Re} \lambda>0)
$$

is a solution of the linearized system, then $\lambda$ is real.

(iii) If $\mathscr{L}^{0} \nsupseteq 0$, denote by $-\alpha^{2}$ the lowest eigenvalue of the operator $\mathscr{L}^{0}$. Then the maximal growth rate $\lambda$ cannot exceed $\alpha$.

Theorem 1.1(i) gives us the linear stability criterion $\mathscr{L}^{0} \geq 0$. In physics, it is a practical and important problem to study $\sigma$-stability ([7]): the equilibrium is said to be $\sigma$-stable if the growth rate does not exceed $\sigma$. Theorem 1.1 (iii) asserts that $\sigma \leq \alpha$ for a Vlasov plasma. We can also compare Theorem 1.1 with the classical energy principle for the stability of a static equilibrium in ideal MHD ([6], [7]), for which the conclusions are rather similar. So Theorem 1.1 can be regarded as the analogue of the energy principle for the case of a symmetric Vlasov plasma. 
We note that the projection $\mathscr{P}$ in the definition of $\mathscr{L}^{0}$ is a highly nonlocal operator since $\mathscr{P} h(x, v)$ turns out to be essentially the average of $h$ in the phase space occupied by the particle trajectory in the steady field $\left(\mathbf{E}^{0}, \mathbf{B}^{0}\right)$ starting at $(x, v)$. So our stability criterion $\mathscr{L}^{0} \geq 0$ is also highly nonlocal, which reflects the collective nature of Vlasov instabilities as mentioned above. In [10], Y. Guo considered the stability of a two-species plasma satisfying 3D RVM without external fields, in a bounded domain with perfectly conducting boundary condition. In a similar setting to ours, a sufficient condition for stability was obtained in [10] by the energy-Casimir method. Extending the calculations in [10] to the whole space case, we obtain the stability condition that $L^{0}>0$ where $L^{0}$ is the local operator

$$
L^{0}=-\partial_{z z}-\partial_{r r}-\frac{1}{r} \partial_{r}+\frac{1}{r^{2}}-r \int \hat{v}_{\theta} \mu_{p} d v .
$$

Since $\mathscr{L}^{0}>L^{0}$, the stability criterion $\mathscr{L}^{0} \geq 0$ in our Theorem 1.1 is a significant improvement because of the additional stabilizing effects from the nonlocal terms in $\mathscr{L}^{0}$. More importantly, in the simpler $1 \frac{1}{2} \mathrm{D}$ case discussed below, we will show that these nonlocal stabilizing terms are indispensable to prove the stability of any equilibrium, even a homogeneous one. We believe that the nonlocal stabilizing terms must also play an important role in plasma stability in the $3 \mathrm{D}$ case.

THE $1 \frac{1}{2}$ D CASE. The simplest case that permits a magnetic field is the socalled $1 \frac{1}{2}$ dimensional case. In this case, physical space is one-dimensional $x \in \mathbb{R}$ and the momentum is two-dimensional $v=\left(v_{1}, v_{2}\right) \in \mathbb{R}^{2}$. Moreover, $\mathbf{E}=\left(E_{1}, E_{2}, 0\right)$ and $\mathbf{B}=(0,0, B)$. We refer to [28] for astrophysical applications of this system. Assuming no external field and setting all physical constants to be 1, system (1.1) reduces to the following $1 \frac{1}{2}$ RVM system

$$
\begin{gathered}
\partial_{t} f^{ \pm}+\hat{v}_{1} \partial_{x} f^{ \pm} \pm\left(E_{1}+\hat{v}_{2} B\right) \partial_{v_{1}} f^{ \pm} \pm\left(E_{2}-\hat{v}_{1} B\right) \partial_{v_{2}} f^{ \pm}=0 \\
\partial_{t} E_{1}=-j_{1}, \quad \partial_{t} E_{2}+\partial_{x} B=-j_{2} \\
\partial_{t} B=-\partial_{x} E_{2}, \quad \partial_{x} E_{1}=\rho
\end{gathered}
$$

with

$$
\rho=\int\left(f^{+}-f^{-}\right) d v, \quad j_{i}=\int \hat{v}_{i}\left(f^{+}-f^{-}\right) d v(i=1,2) .
$$

The main reason for considering $1 \frac{1}{2} \mathrm{D}$ RVM is its simplicity, and yet it preserves many of the essential features of 3D RVM. So we expect that a good understanding of the $1 \frac{1}{2} \mathrm{D}$ case can provide us new tools and insights in the 3D case. We also note that the global existence of classical solutions of $1 \frac{1}{2} \mathrm{D}$ RVM is known [17], while the global existence of classical solutions and the uniqueness of weak solutions of 3D RVM are still open questions.

We consider solutions of the system (1.9) that are periodic in the variable $x$ with a given period $P$. Consider the $P$-periodic equilibrium

$$
f^{0, \pm}=\mu^{ \pm}\left(e^{ \pm}, p^{ \pm}\right)=\mu^{ \pm}\left(\langle v\rangle \pm \phi^{0}(x), v_{2} \pm \psi^{0}(x)\right),
$$


and

$$
E_{1}^{0}=-\partial_{x} \phi^{0}, \quad E_{2}^{0}=0, \quad B^{0}=\partial_{x} \psi^{0},
$$

where $\left(\phi^{0}, \psi^{0}\right)$ satisfy the ODE system

$$
\partial_{x}^{2} \phi^{0}=-\rho^{0}=-\int\left(f^{0,+}-f^{0,-}\right) d v, \quad \partial_{x}^{2} \psi^{0}=-j_{2}^{0}=-\int \hat{v}_{2}\left(f^{0,+}-f^{0,-}\right) d v .
$$

In the appendix we show that there exist infinitely many periodic electromagnetic equilibria of the above form. We assume that

$$
\mu^{ \pm} \geq 0, \mu^{ \pm} \in C^{1}, \mu_{e}^{ \pm}<0,\left|\mu_{e}^{ \pm}\right|+\left|\mu_{p}^{ \pm}\right| \leq c(1+|e|)^{-\alpha}
$$

for some $\alpha>2$.

We denote

$$
\begin{gathered}
D^{ \pm}=\hat{v}_{1} \partial_{x} \pm\left(E_{1}^{0}+\hat{v}_{2} B^{0}\right) \partial_{v_{1}} \mp \hat{v}_{1} B^{0} \partial_{v_{2}} \\
L_{\left|\mu_{e}^{ \pm}\right|}^{2}=\left\{f \mid f \text { periodic in } x,\|f\|_{ \pm}^{2} \equiv \int_{0}^{P} \int_{-\infty}^{\infty}|f|^{2}\left|\mu_{e}^{ \pm}\right| d v d x<\infty\right\}
\end{gathered}
$$

and $\mathscr{P}^{ \pm}=$the projection operator of $L_{\left|\mu_{e}^{ \pm}\right|}^{2}$ onto $\operatorname{ker} D^{ \pm}$. We define the following operators acting on $L_{P}^{2}(\mathbb{R})$, where the subscript $P$ refers to the periodicity.

$$
\begin{gathered}
\mathscr{A}_{1}^{0} h=-\partial_{x}^{2} h-\left(\sum_{ \pm} \int \mu_{e} d v\right) h+\sum_{ \pm} \int \mu_{e}^{ \pm} \mathscr{P}^{ \pm} h d v . \\
\mathscr{A}_{2}^{0} h=-\partial_{x}^{2} h-\left(\sum_{ \pm} \int \hat{v}_{2} \mu_{p}^{ \pm} d v\right) h-\sum_{ \pm} \int \mu_{e}^{ \pm} \hat{v}_{2} \mathscr{P}^{ \pm}\left(\hat{v}_{2} h\right) d v . \\
\mathscr{B}^{0} h=\left(\sum_{ \pm} \int \mu_{p}^{ \pm} d v\right) h+\sum_{ \pm} \int \mu_{e}^{ \pm} \mathscr{P}^{ \pm}\left(\hat{v}_{2} h\right) d v
\end{gathered}
$$

and

$$
\mathscr{L}^{0}=\left(\mathscr{B}^{0}\right)^{*}\left(\mathscr{A}_{1}^{0}\right)^{-1} \mathscr{B}^{0}+\mathscr{A}_{2}^{0}
$$

As in the $3 \mathrm{D}$ case, we have the following result in $1 \frac{1}{2} \mathrm{D}$.

Theorem 1.2. Consider the $1 \frac{1}{2} D$ case under the assumptions (1.12). Then

(i) $\mathscr{L}^{0} \geq 0$ implies spectral stability.

(ii) Any growing mode must be purely growing.

(iii) Denote by $-\alpha^{2}$ the lowest eigenvalue of the operator $\mathscr{L}^{0}$. The maximal growth rate cannot exceed $\alpha$.

Moreover, we have the following additional theorem asserting the existence of growing modes in $1 \frac{1}{2} \mathrm{D}$ and showing that the condition $\mathscr{L}^{0} \geq 0$ is sharp.

Theorem 1.3. If (1.12) holds, and $\psi^{0}, \phi^{0}$ are even in $[0, P]$, and $\mathscr{L}^{0}$ has an even eigenfunction corresponding to a negative eigenvalue, then there exists a real growing mode $\left[e^{\lambda t} f(x, v), e^{\lambda t} E(x), e^{\lambda t} B(x)\right]$ with $f, E, B \in W^{1,1}$ and $\lambda>0$. 
Theorem 1.3 is applicable for instance to the even periodic equilibrium constructed in the appendix. In Section 4, we show that if $\mathscr{L}^{0} \nsucceq 0$ for a homogeneous equilibrium or a purely magnetic equilibrium $\left(\phi^{0}=0\right)$, then there indeed exists an even eigenfunction corresponding to a negative eigenvalue. Thus combining Theorems 1.2 and 1.3 , we get the sharp stability criterion $\mathscr{L}^{0} \geq 0$ for any homogeneous equilibrium or purely magnetic equilibrium.

In Section 4, we construct various stable and unstable $1 \frac{1}{2} \mathrm{D}$ examples using our criterion. First we show that any homogeneous equilibrium is either stable to perturbations of arbitrary period or stable only up to a critical period. This improves the results of [15] where the homogeneous equilibria are assumed to be even in $v_{2}$. In particular, in [15] stability for subcritical period is only proven under the implicit assumption that the perturbed magnetic field is even. Our result removes this restriction by utilizing the stabilizing nonlocal term in $\mathscr{L}^{0}$. Perturbing from the unstable homogeneous equilibrium, we can easily construct weakly inhomogeneous purely magnetic equilibria that are unstable under perturbations of double the period. It is more interesting that we construct an inhomogeneous purely magnetic example that is stable under perturbations of minimum period but becomes unstable under perturbations of double the period. Note that for homogeneous equilibria the perturbations of longer wavelength are more unstable (by Theorem 4.1 in Section 4). So our example shows that for the RVM system long waves might be always more unstable, even for inhomogeneous equilibria. The proof of stability for inhomogeneous equilibria is rather tricky and the nonlocal stabilizing term in $\mathscr{L}^{0}$ plays the crucial role once again. In our subsequent paper [25], we will show that the linear stability and instability results stated above are also true on the nonlinear dynamical level.

We now sketch the main ideas in the proofs of these results. To prove the spectral stability in Theorems 1.1 and 1.2, we start by finding all the invariants of the linearized RVM system. The first invariant $I$ corresponds to the quadratic term of the Taylor expansion of the usual energy-Casimir functional introduced in the nonlinear stability analysis. The second set of invariants, which is due to the special structure of the linearized equation, is less obvious. For the $3 \mathrm{D}$ case the invariants are

$$
K_{g}=\iint\left(f+\mu_{e} \hat{v} \cdot \mathbf{A}+r \mu_{p} A_{\theta}\right) g d x d v
$$

for all $g \in \operatorname{ker} D$, where $\mathbf{A}$ is the magnetic potential. For a growing mode, all the invariants $I$ and $K_{g}$ must vanish. We then use the same strategy as in the study of spectral stability of ideal plane Euler flows ([21]), namely, to minimize $I$ under the constraints that $K_{g}=0$ for all $g \in \operatorname{ker} D$. This minimization problem is rather delicate for 3D RVM. Nevertheless, we are able to show that $\mathscr{L}^{0} \geq 0$ implies that the constrained minimum $I_{\min }$ is positive, which excludes the existence of any growing mode. 
To estimate the maximal growth rate and prove the purely growing property, we borrow some ideas from stellar dynamics and MHD. Recall that to derive the MHD energy principle one reduces the linearized MHD system to the second order system

$$
\rho \frac{d^{2} \xi}{d t^{2}}=F \xi
$$

where $F$ is self-adjoint and $\rho>0$. Then the purely growing property follows immediately. The spectral stability and the estimate of the growth rate follows ([19]) from the two identities

$$
\frac{d}{d t}(K-W)=0 \text { and } \frac{d^{2}}{d t^{2}} N=K+W
$$

where

$$
K=(\rho \dot{\xi}, \dot{\xi}), N=\frac{1}{2}(\rho \xi, \xi), W=(F \xi, \xi) .
$$

On the other hand, the linearized RVM system is a first-order system and there is no easy way to reduce it to a second-order system of the form (1.17). In the study of stellar stability modeled by 3D Vlasov-Poisson, Antonov introduced an elegant splitting argument which allowed him to write the linearized Vlasov-Poisson system in the form (1.17) and to derive his famous energy principle for stellar stability ([2]). We use a similar splitting idea here. For the $1 \frac{1}{2} \mathrm{D}$ case, the splitting is $f=f_{e v}+f_{o d}$ with $f_{e v}=\frac{1}{2}\left(f\left(x, v_{1}, v_{2}\right)+f\left(x,-v_{1}, v_{2}\right)\right)$. Unlike the Vlasov-Poisson case, the linearized RVM system cannot be written in the form (1.17) by this splitting. However, by lengthy calculations we are able to derive two identities of the form (1.18); see Lemmas 2.9 and 2.10. This allows us to prove spectral stability and to control the maximal growth rate. But we cannot obtain the existence of a exponentially growing solution as in the usual energy principle for (1.17) because the linearized RVM is not a second-order system.

In order to construct growing modes, we use the method of dispersion operators together with continuation, an argument first introduced in [20] for the 1D VlasovPoisson system. In [20] the idea is to express $f$ in terms of the electric potential $\phi$ by integrating the Vlasov equation along the particle trajectory, then to plug $f$ into the Poisson equation to get a self-adjoint dispersion operator for $\phi$, and finally to use a continuation argument to get growing modes. There are several difficulties in extending this approach to RVM models. In the $1 \frac{1}{2} \mathrm{D}$ case, assume the growing mode has periodic electromagnetic potentials $(\phi, \psi)$, and express $f$ in term of them by integration along the trajectory. Plugging $f$ into the Maxwell system, we unfortunately do not get a self-adjoint problem for $(\phi, \psi)$. However, using the condition $\mu_{e}<0$, we can eliminate $\phi$ to get a self-adjoint dispersion operator for $\psi$ alone and can then apply the continuation argument as in [20]. One difficulty with this approach is that the equation

$$
\partial_{t} E_{1}=-j_{1}
$$


(part of Maxwell's) is not taken care of by the dispersion operators. For the $1 \frac{1}{2} \mathrm{D}$ RVM system (1.9), equation (1.19) and the Poisson equation

$$
\partial_{x} E_{1}=\rho
$$

are somewhat redundant but not equivalent. To derive (1.19) from (1.20), it is enough to check that both $E_{1}$ and $j_{1}$ have zero mean. Under the assumption of Theorem 1.3, we show by means of a parity argument that if $\psi$ is even, then $j_{1}$ indeed has zero mean. Then (1.19) is satisfied and we get a growing mode. In the 3D case, an analogous difficulty exists with the dispersion operator approach. However, the difficulty cannot be solved by a parity argument similar to the $1 \frac{1}{2} \mathrm{D}$ case and we have not succeeded in handling it.

This paper is organized as follows. In Section 2, we prove Theorems 1.2 and 1.3. To simply our notation, we only give the proof for the single species case. In Section 3, we prove Theorem 1.1 in the 3D case. In Section 4 , we discuss the $1 \frac{1}{2} \mathrm{D}$ stability criterion for the special cases of homogeneous and of purely magnetic equilibria. In particular, a stable inhomogeneous example is explicitly constructed. In the appendix we show that there exists infinitely many periodic electromagnetic equilibria in $1 \frac{1}{2} \mathrm{D}$. With properly chosen external fields, a confined equilibrium with support in a torus is constructed.

\section{Linear stability of periodic $1 \frac{1}{2}$ dimensional RVM}

In the following discussions of stability (until the end of this section), with the sole purpose of simplifying our notation, we consider a constant ion background $n_{0}$. All the proofs remain almost unchanged for the more general two-species case. The $1 \frac{1}{2} \mathrm{D}$ RVM for one species becomes

$$
\begin{gathered}
\partial_{t} f+\hat{v}_{1} \partial_{x} f-\left(E_{1}+\hat{v}_{2} B\right) \partial_{v_{1}} f-\left(E_{2}-\hat{v}_{1} B\right) \partial_{v_{2}} f=0 \\
\partial_{t} E_{1}=-j_{1}=\int \hat{v}_{1} f d v, \partial_{t} B=-\partial_{x} E_{2} \\
\partial_{t} E_{2}+\partial_{x} B=-j_{2}=\int \hat{v}_{2} f d v
\end{gathered}
$$

with the constraint

$$
\partial_{x} E_{1}=n_{0}-\int f d v
$$

Fixing any such equilibrium with a period $P$, we will consider the system (21) with periodic boundary conditions of the same period $P$.

The equilibrium is assumed to have the form $f^{0}=\mu(e, p), E_{1}^{0}=-\partial_{x} \phi^{0}, E_{2}^{0}=$ $0, B^{0}=\partial_{x} \psi^{0}$, where the electromagnetic potentials $\left(\phi^{0}, \psi^{0}\right)$ satisfy the ODE system

$$
\partial_{x}^{2} \phi^{0}=n_{0}-\int \mu(e, p) d v, \quad \partial_{x}^{2} \psi^{0}=\int \hat{v}_{2} \mu(e, p) d v
$$


with the electron energy and the "angular momentum" defined by

$$
e=\langle v\rangle-\phi^{0}(x), \quad p=v_{2}-\psi^{0}(x) .
$$

(The $e$ is distinguished from the exponential $e$ in context.) The only assumptions we make on $\mu$ are

$$
\mu \geq 0, \quad \mu \in C^{1}, \quad \mu_{e} \equiv \frac{\partial \mu}{\partial e}<0
$$

and, in order for $\int\left(\left|\mu_{e}\right|+\left|\mu_{p}\right|\right) d v$ to be finite,

$$
\left(\left|\mu_{e}\right|+\left|\mu_{p}\right|\right)(e, p) \leq c(1+|e|)^{-\alpha} \text { for some } \alpha>2 .
$$

The linearized evolution equations are

$$
\left(\partial_{t}+D\right) f=\mu_{e} \hat{v}_{1} E_{1}-\mu_{p} \hat{v}_{1} B+\left(\mu_{e} \hat{v}_{2}+\mu_{p}\right) E_{2},
$$

where $D$ is the transport operator associated with the steady fields,

$$
\begin{aligned}
D & =\hat{v}_{1} \partial_{x}-\left(E_{1}^{0}+\hat{v}_{2} B^{0}\right) \partial_{v_{1}}+\hat{v}_{1} B^{0} \partial_{v_{2}} \\
& =\hat{v}_{1} \partial_{x}+\partial_{x} \phi^{0} \partial_{v_{1}}+\partial_{x} \psi^{0}\left(\hat{v}_{1} \partial_{v_{2}}-\hat{v}_{2} \partial_{v_{1}}\right),
\end{aligned}
$$

together with

$$
\partial_{x} E_{1}=-\int f d v, \partial_{t} E_{1}=\int \hat{v}_{1} f d v, \partial_{t} E_{2}+\partial_{x} B=\int \hat{v}_{2} f d v, \partial_{t} B+\partial_{x} E_{2}=0
$$

We define the Hilbert space

$$
L_{\left|\mu_{e}\right|}^{2}=\left\{f(x, v) \mid f \text { periodic in } x,\|f\|_{\left|\mu_{e}\right|}^{2} \equiv \int_{0}^{P} \int_{-\infty}^{\infty}|f|^{2}\left|\mu_{e}\right| d v d x<\infty\right\}
$$

and denote its inner product by $(\cdot, \cdot)_{\left|\mu_{e}\right|}$. Let $\mathscr{P}$ be the projection operator of $L_{\left|\mu_{e}\right|}^{2}$ onto the kernel of $D$. We also denote by $L_{P}^{p}\left(H_{P}^{2}\right)$ the space of $P$-periodic $L_{x}^{p}\left(H_{x}^{2}\right)$ functions for $p \geq 1$.

Similarly to the two-species case, we define the following four operators, each of which acts from $H_{P}^{2}$ to $L_{P}^{2}$,

$$
\begin{gathered}
\mathscr{A}_{1}^{0} h=-\partial_{x}^{2} h-\left(\int \mu_{e} d v\right) h+\int \mu_{e} \mathscr{P} h d v, \\
\mathscr{A}_{2}^{0} h=-\partial_{x}^{2} h-\left(\int \hat{v}_{2} \mu_{p} d v\right) h-\int \mu_{e} \hat{v}_{2} \mathscr{P}\left(\hat{v}_{2} h\right) d v, \\
\mathscr{B}^{0} h=\left(\int \mu_{p} d v\right) h+\int \mu_{e} \mathscr{P}\left(\hat{v}_{2} h\right) d v
\end{gathered}
$$

and

$$
\mathscr{L}^{0}=\left(\mathscr{B}^{0}\right)^{*}\left(\mathscr{A}_{1}^{0}\right)^{-1} \mathscr{B}^{0}+\mathscr{A}_{2}^{0} .
$$

In these definitions one should keep in mind that $\mu \geq 0$ is a function of $x$ and $v$, that $\mu_{e}=\partial \mu / \partial e<0$ and that $\mu_{p}=\partial \mu / \partial p$. We will show in Lemma 2.4 that $\mathscr{A}_{1}^{0}$ is invertible on the range of $\mathscr{B}^{0}$ so that $\mathscr{L}^{0}$ will be well-defined. 
By spectral stability of the system (2.1) with respect to the given equilibrium, we mean that there is no exponentially growing mode

$$
\left(e^{\lambda t} f_{g}(x, v), e^{\lambda t} E_{g}(x), e^{\lambda t} B_{g}(x)\right)
$$

with $\operatorname{Re} \lambda>0$ of the linearized equations (2.6), (2.8) which has period $P$ in $x$, where $E_{g}, B_{g} \in L_{P}^{2}$ and $f_{g} \in L_{x, v}^{1}$. On the other hand, if there exists such a growing mode, the system is called spectrally unstable. The following two theorems are just the one-species versions of Theorems 1.2 and 1.3.

Theorem 2.1. Assume (2.4) and (2.5).

(i) The system is spectrally stable if $\mathscr{L}^{0} \geq 0$ as an self-adjoint operator on $L_{P}^{2}$.

(ii) If $\left[e^{\lambda t} f_{g}(x, v), e^{\lambda t} E_{g}(x), e^{\lambda t} B_{g}(x)\right]$ is a growing mode, then $\lambda$ is real.

(iii) The maximal growth rate $\lambda$ of a growing mode cannot exceed $\alpha$, where $-\alpha^{2}$ is the lowest eigenvalue of $\mathscr{L}^{0}$.

For the existence of growing modes, we consider a periodic equilibrium with even $\psi^{0}$ and $\phi^{0}$. The simplest example is a purely growing magnetic equilibrium with $\phi^{0}=0$, in which case $\psi^{0}$ satisfies a second order ODE and can always be assumed to be even by adjusting its starting point. In the appendix we will show that there exist infinitely many periodic, even electromagnetic equilibria in the case of two species.

Theorem 2.2. Assume (2.4), (2.5) and that $\psi^{0}, \phi^{0}$ are even. If $\mathscr{L}^{0}$ has an even eigenfunction corresponding to a negative eigenvalue, then there exists a growing mode $\left[e^{\lambda t} f_{g}(x, v), e^{\lambda t} E_{g}(x), e^{\lambda t} B_{g}(x)\right]$ with $f \in W_{x, v}^{1,1}$ and $(E, B) \in W_{P}^{1,1}$.

\subsection{The Operators}

We begin by discussing the operator $D$.

Lemma 2.3. $D$ is a skew-adjoint operator on $L_{\left|\mu_{e}\right|}^{2}$. Its nullspace $\operatorname{ker} D$ consists of all functions $g=g(x, v)$ in $L_{\left|\mu_{e}\right|}^{2}$ that are constant on each connected component in $\mathbb{R} \times \mathbb{R}^{2}$ of $\left\{e=\langle v\rangle-\phi^{0}(x)=\right.$ constant and $p=v_{2}-\psi^{0}(x)=$ constant $\}$. In particular, $\operatorname{ker} D$ contains all functions of $e$ and $p$.

Proof. The skew-adjointness follows from an integration by parts. The domain of $D$ is $\left\{f \in L_{\left|\mu_{e}\right|}^{2} \mid D f \in L_{\left|\mu_{e}\right|}^{2}\right\}$. The characteristics of $D$ are given by the ODEs

$$
\dot{X}=\hat{V}_{1}, \quad \dot{V}_{1}=\partial_{x} \phi^{0}(X)-\hat{V}_{2} \partial_{x} \psi^{0}(X), \quad \dot{V}_{2}=\hat{V}_{1} \partial_{x} \psi^{0}(X)
$$

We verify that $D e=0$ and $D p=0$ and then use the chain rule.

We also introduce the particle paths $(X(t ; x, v), V(t ; x, v))$, which are the characteristics of $D$. They are defined as the solutions of (2.9) with the initial conditions $X(0)=x, V(0)=v$. Now we introduce some additional operators depending on a parameter $\lambda$. For any $\lambda$ with $\operatorname{Re} \lambda>0$, we define the following three operators

$$
\mathscr{A}_{1}^{\lambda} h=-\partial_{x}^{2} h-\left(\int \mu_{e} d v\right) h+\int \mu_{e} \int_{-\infty}^{0} \lambda e^{\lambda s} h(X(s)) d s d v
$$




$$
\begin{gathered}
\mathscr{A}_{2}^{\lambda} h=-\partial_{x}^{2} h+\lambda^{2} h-\left(\int \hat{v}_{2} \mu_{p} d v\right) h-\int \hat{v}_{2} \mu_{e} \int_{-\infty}^{0} \lambda e^{\lambda s} \hat{V}_{2}(s) h(X(s)) d s d v, \\
\mathscr{B}^{\lambda} h=\left(\int \mu_{p} d v\right) h+\int \mu_{e} \int_{-\infty}^{0} \lambda e^{\lambda s} \hat{V}_{2}(s) h(X(s)) d s d v .
\end{gathered}
$$

We will show that $\mathscr{A}_{1}^{\lambda}$ is invertible on the range of $\mathscr{B}^{\lambda}$, so that the operator

$$
\mathscr{L}^{\lambda}=\left(\mathscr{B}^{\lambda}\right)^{*}\left(\mathscr{A}_{1}^{\lambda}\right)^{-1} \mathscr{B}^{\lambda}+\mathscr{A}_{2}^{\lambda} .
$$

is also well-defined.

Lemma 2.4. Assume $\lambda>0$.

(i) The operators $\mathscr{A}_{j}^{\lambda}, \mathscr{A}_{j}^{0}, \mathscr{L}^{\lambda}$ and $\mathscr{L}^{0}(j=1,2)$ are self-adjoint on $L_{P}^{2}$ with the common domain $H_{P}^{2}$. Their spectra are discrete.

(ii) $\mathscr{A}_{1}^{\lambda} \geq 0$ for all $\lambda \geq 0$.

(iii) The nullspace $N\left(\mathscr{A}_{1}^{\lambda}\right)$ consists of the constant functions. The inverse $\left(\mathscr{A}_{1}^{\lambda}\right)^{-1}$ is bounded from $\left\{h \in L_{P}^{2} \mid \int_{0}^{P} h d x=0\right\}=N\left(\mathscr{A}_{1}^{\lambda}\right)^{\perp} \supset R\left(\mathscr{B}^{\lambda}\right)$ into $H_{P}^{2}$.

(iv) If $\psi^{0}, \phi^{0}$ are even functions, then the operators $\mathscr{A}_{j}^{\lambda}, \mathscr{A}_{j}^{0}, \mathscr{L}^{\lambda}$ and $\mathscr{L}^{0}$ preserve $\operatorname{parity}(j=1,2)$.

Proof. We first claim that $\mathscr{A}_{j}^{\lambda}+\partial_{x}^{2}$ and $\mathscr{B}^{\lambda}$ are bounded operators on $L_{P}^{2}$ for all $\lambda \geq 0$. For instance, for $\lambda=0$ a typical one of these operators $L$ is estimated as

$$
(L h, k)=\iint \mu_{e} \mathscr{P} h d v \cdot k d x \leq\left(\int \sup _{x}\left|\mu_{e}\right| d v\right)\|h\|\|k\| \leq C\|h\|\|k\|
$$

by the decay assumption on $\mu_{e}$. For $\lambda>0$, a typical one of these operators $L$ is given by

$$
(L h, k)=\iiint \lambda e^{\lambda s} \mu_{e} h(X(s)) k(x) d s d v d x .
$$

We use Schwarz' inequality and then, in the factor involving $h(X(s))$, we use the change of variables $x, v \mapsto X, V$ which has Jacobian $=1$. Thereby we obtain

$$
(L h, k) \leq\left(\int \sup _{x}\left|\mu_{e}\right| d v\right)\|h\|\|k\| \leq C\|h\|\|k\| .
$$

The other operators $L$ have extra factors of $\hat{v}_{2}$ and $\hat{V}_{2}$, which do not change the estimates.

Secondly, we prove the symmetry of the operators $\mathscr{A}$. Of course, $-\partial_{x}^{2}$ is symmetric (and unbounded). In order to prove the symmetry of $\mathscr{A}_{2}^{0}$, we notice that

$$
\iint \hat{v}_{2} \mu_{e} \mathscr{P}\left(\hat{v}_{2} h\right) d v \cdot k d x=\iint \mu_{e} \mathscr{P}\left(\hat{v}_{2} h\right) \mathscr{P}\left(\hat{v}_{2} k\right) d v d x
$$


which is clearly symmetric in $h$ and $k$, because of the definition of $\mathscr{P}$. In order to prove the symmetry of $\mathscr{A}_{2}^{\lambda}$, we calculate

$$
\begin{aligned}
& \iiint \lambda e^{\lambda s} \hat{v}_{2} \mu_{e} \hat{V}_{2}(s ; x, v) h(X(s ; x, v)) d s d v \cdot k(x) d x \\
& =\iiint \lambda e^{\lambda s} \mu_{e} h(y) \hat{w}_{2} \hat{V}_{2}(-s ; y, w) k(X(-s ; y, w)) d s d w d y,
\end{aligned}
$$

where we have changed variables $y=X(s ; x, v), w=V(s ; x, v)$ and used the invariance of $e$ and $p$ and hence of $\mu_{e}$. Notice that $X\left(-s ; y,-w_{1}, w_{2}\right)=X\left(s ; y, w_{1}, w_{2}\right)$, $-V_{1}\left(-s ; y,-w_{1}, w_{2}\right)=V_{1}\left(s ; y, w_{1}, w_{2}\right)$ and $V_{2}\left(-s ; y,-w_{1}, w_{2}\right)=V_{2}\left(s ; y, w_{1}, w_{2}\right)$. So we can change $w_{1} \mapsto-w_{1}, w_{2} \rightarrow+w_{2}, y \mapsto+y$ to obtain

$$
\iiint \lambda e^{\lambda s} \mu_{e} h(y) \hat{w}_{2} \hat{V}_{2}(s ; y, w) k(X(s ; y, w)) d s d w d y .
$$

If we replace $y, w$ by $x, v$, this is the symmetric expression that we desired. The symmetry of $\mathscr{A}_{1}^{\lambda}$ is proven in the same way by omitting the $\hat{v}$ factors.

However, the operators $\mathscr{B}^{\lambda}$ are not symmetric. But by almost the same calculation, we find the formula for the adjoint of $\mathscr{B}^{\lambda}$ as

$$
\begin{aligned}
\mathscr{B}^{\lambda *} h & =\int \mu_{p} d v h+\int \hat{v}_{2} \mu_{e} \int_{-\infty}^{0} \lambda e^{\lambda s} h(X(s)) d s d v \\
& =\int \hat{v}_{2} \mu_{e}\left\{-h+\int_{-\infty}^{0} \lambda e^{\lambda s} h(X(s)) d s\right\} .
\end{aligned}
$$

Thirdly, we prove the discreteness of the spectra of the $\mathscr{A}_{j}^{\lambda}$. All of the bounded operators discussed above are relatively compact, that is, relative to the operator $\partial_{x}^{2}$. By the Kato-Rellich Theorem, $\mathscr{A}_{j}^{\lambda}$ are self-adjoint with domain $H_{P}^{2}$ for $j=1,2$ and $\lambda \geq 0$. By Weyl's Theorem, these operators have the same essential spectra as $-\partial_{x}^{2}$. Since the latter has purely discrete spectrum, so do the operators $\mathscr{A}_{j}^{\lambda}$.

Fourthly, we prove (ii). For $\lambda>0$, we have $\left(\mathscr{A}_{1}^{\lambda} h, h\right)=I+I I+I I I$ where $I=\int\left(\partial_{x} h\right)^{2} d x \geq 0, I I=\iint\left|\mu_{e}\right| h^{2} d v d x \geq 0$ and

$$
\begin{aligned}
|I I I| & \leq \iiint \lambda e^{\lambda s}\left|\mu_{e}\right||h(X(s))||h(x)| d s d v d x \\
& \leq \int \lambda e^{\lambda s}\left\{\iint\left|\mu_{e}\right||h(X(s))|^{2} d v d x\right\}^{\frac{1}{2}}\left\{\iint\left|\mu_{e}\right||h(x)|^{2} d v d x\right\}^{\frac{1}{2}} d s \\
& =\int_{-\infty}^{0} \lambda e^{\lambda s} d s \iint\left|\mu_{e}\right||h(x)|^{2} d v d x=I I .
\end{aligned}
$$

Thus $\left(\mathscr{A}_{1}^{\lambda} h, h\right) \geq \int\left(\partial_{x} h\right)^{2} d x \geq 0$ for all $h \in L_{P}^{2}$. In case $\lambda=0$, we write $\mathscr{A}_{1}^{0}=$ $I+I I+I I I$ in the same way and estimate

$$
|I I I|=\iint\left|\mu_{e}\right||\mathscr{P}(h)|^{2} d v d x \leq \iint\left|\mu_{e}\right||h|^{2} d v d x=I I .
$$

This proves (ii). 
Fifthly, we prove (iii). By the inequality just above, $N\left(\mathscr{A}_{1}^{\lambda}\right) \subset\{$ constants $\}$. On the other hand, a direct calculation shows that $\mathscr{A}_{1}^{\lambda} 1=0$ for all $\lambda \geq 0$. Thus $N\left(\mathscr{A}_{1}^{\lambda}\right)=\{$ constants $\}$ and $1^{\perp}=\left\{h \in L_{P}^{2} \mid \int_{0}^{P} h d x=0\right\}$. Since the spectrum of $\mathscr{A}_{1}^{\lambda}$ is discrete, it follows that the restriction of $\mathscr{A}_{1}^{\lambda}$ to $1^{\perp}$ is invertible. Now it is easy to see that $R\left(\mathscr{B}^{\lambda}\right) \subset 1^{\perp}$. Indeed, for $\lambda>0$,

$$
\begin{aligned}
\int_{0}^{P} \mathscr{B}^{\lambda} h d x & =-\iint \hat{v}_{2} \mu_{e} h d v d x+\iiint \mu_{e} \lambda e^{\lambda s} \hat{V}_{2} h(X(s)) d s d v d x \\
& =-\iint \hat{v}_{2} \mu_{e} h d v d x+\iiint \mu_{e} \lambda e^{\lambda s} \hat{v}_{2} h(x) d s d v d x=0 .
\end{aligned}
$$

A similar proof is valid in the case $\lambda=0$. This proves (iii). The self-adjointness and discrete spectrum of $\mathscr{L}^{\lambda}$ for any $\lambda \geq 0$ follows immediately for the same reasons as above.

Finally, to prove (iv), first we observe that if $\psi^{0}, \phi^{0}$ are even in $[0, P]$, then

$\left(X\left(t ; P-x,-v_{1}, v_{2}\right), V\left(t ; P-x,-v_{1}, v_{2}\right)\right)=\left(P-X(t ; x, v),-V_{1}(t ; x, v), V_{2}(t ; x, v)\right)$.

So if $h$ is even, then for a typical nonlocal term appeared in the definition of $\mathscr{A}_{j}^{\lambda}, \mathscr{B}^{\lambda}, \mathscr{L}^{\lambda}$

$$
\mathscr{K} h=\int \mu_{e} \int_{-\infty}^{0} \lambda e^{\lambda s} h(X(s)) d s d v
$$

we have

$$
\begin{aligned}
\mathscr{K} h(P-x) & =\int \mu_{e} \int_{-\infty}^{0} \lambda e^{\lambda s} h\left(X\left(s ; P-x,-v_{1}, v_{2}\right)\right) d s d v \\
& =\int \mu_{e} \int_{-\infty}^{0} \lambda e^{\lambda s} h(P-X(s ; x, v)) d s d v(\text { by }(2.11)) \\
& =\int \mu_{e} \int_{-\infty}^{0} \lambda e^{\lambda s} h(X(s ; x, v)) d s d v=\mathscr{K} h(x)
\end{aligned}
$$

By the same calculation, $\mathscr{K} h$ is odd is $h$ is odd, so $\mathscr{K}$ preserves parity. Similarly, we can show that the operators $\mathscr{A}_{j}^{\lambda}, \mathscr{L}^{\lambda}$ preserve parity. By the proof of Lemma 2.6 below, $\mathscr{A}_{j}^{\lambda}, \mathscr{B}^{\lambda}, \mathscr{L}^{\lambda}$ converge to $\mathscr{A}_{j}^{0}, \mathscr{B}^{0}, \mathscr{L}^{0}$ strongly, so $\mathscr{A}_{j}^{0}, \mathscr{B}^{0}, \mathscr{L}^{0}$ also preserve parity.

The following lemma explains why we introduced the operators discussed above.

Lemma 2.5. Assume that $\psi^{0}, \phi^{0}$ are even periodic functions. If $\mathscr{L}^{\lambda}$ has a nontrivial nullspace of even functions for some $\lambda>0$, then there exists a purely growing mode of (2.6), (2.8).

Proof. Assume $0 \neq \psi \in H_{P}^{2}$ is such that $\mathscr{L}^{\lambda} \psi=0$ and $\psi$ is even. We define $\phi=\left(\mathscr{A}_{1}^{\lambda}\right)^{-1} \mathscr{B}^{\lambda} \psi$ then clearly $\phi \in H_{P}^{2}$ and $\phi$ is even by Lemma 2.4. Define $f$ as

$$
f(x, v)=-\mu_{e} \phi(x)-\mu_{p} \psi(x)+\mu_{e} \int_{-\infty}^{0} \lambda e^{\lambda s}\left[\phi(X(s))-\hat{V}_{2}(s) \psi(X(s))\right] d s
$$


From (2.12) it is obvious that $f \in L_{x, v}^{2} \cap L_{x, v}^{1}$,

$$
\begin{aligned}
\iint f d x d v= & \iint\left(-\mu_{e} \phi(x)-\mu_{p} \psi(x)\right) d x d v \\
& +\int_{-\infty}^{0} \lambda e^{\lambda s} \iint \mu_{e}\left[\phi(X(s))-\hat{V}_{2}(s) \psi(X(s))\right] d x d v d s \\
= & \left.\iint\left(-\mu_{e} \phi(x)-\mu_{p} \psi(x)\right) d x d v+\int_{-\infty}^{0} \lambda e^{\lambda s} \iint \mu_{e}[\phi(x))-\hat{v}_{2} \psi(x)\right] d x d v d s \\
= & \left.\iint\left(-\mu_{e} \phi(x)-\mu_{p} \psi(x)\right) d x d v+\iint \mu_{e}[\phi(x))-\hat{v}_{2} \psi(x)\right] d x d v=0 .
\end{aligned}
$$

We also have $\int j_{1} d x=\iint \hat{v}_{1} f d x d v=0$, since as in the proof of Lemma 2.4 (iv)

$$
\begin{aligned}
& j_{1}(P-x) \\
= & \int \hat{v}_{1} \mu_{e} \int_{-\infty}^{0} \lambda e^{\lambda s}\left[\phi\left(X\left(s ; P-x, v_{1}, v_{2}\right)\right)-\hat{V}_{2}\left(s ; P-x, v_{1}, v_{2}\right) \psi\left(X\left(s ; P-x, v_{1}, v_{2}\right)\right)\right] d s d v \\
= & -\int \hat{v}_{1} \mu_{e} \int_{-\infty}^{0} \lambda e^{\lambda s}\left[\phi\left(X\left(s ; P-x,-v_{1}, v_{2}\right)\right)-\hat{V}_{2}\left(s ; P-x,-v_{1}, v_{2}\right) \psi\left(X\left(s ; P-x,-v_{1}, v_{2}\right)\right)\right] d s d v \\
= & -\int \hat{v}_{1} \mu_{e} \int_{-\infty}^{0} \lambda e^{\lambda s}\left[\phi\left(P-X\left(s ; x, v_{1}, v_{2}\right)\right)-\hat{V}_{2}\left(s ; x, v_{1}, v_{2}\right) \psi\left(P-X\left(s ; x, v_{1}, v_{2}\right)\right)\right] d s d v \\
= & -\int \hat{v}_{1} \mu_{e} \int_{-\infty}^{0} \lambda e^{\lambda s}\left[\phi(X(s))-\hat{V}_{2}(s) \psi(X(s))\right] d s d v=-j_{1}(x) .
\end{aligned}
$$

In [25] we show that although the function $f$ may not be differentiable everywhere, we have $f \in W_{x, v}^{1,1}$. Here we use this fact, which follows by the same proof as in [22]; see also Lemma 5 in [25]. Define

$$
B=\partial_{x} \psi, E_{2}=-\lambda \psi, E_{1}=-\partial_{x} \phi .
$$

We shall show that $\left[e^{\lambda t} f(x, v), e^{\lambda t} E(x), e^{\lambda t} B(x)\right]$ is a growing mode satisfying the linearized system (2.6), (2.8). This is equivalent to checking that $[f(x, v), E(x), B(x)]$ satisfy

$$
\lambda f+D f=-\mu_{e} \hat{v}_{1} \partial_{x} \phi-\mu_{p} \hat{v}_{1} \partial_{x} \psi-\left(\lambda \mu_{e} \hat{v}_{2}+\lambda \mu_{p}\right) \psi
$$

and

$$
\partial_{x} E_{1}=\rho, \lambda E_{1}=-j_{1}, \lambda E_{2}+\partial_{x} B=-j_{2}, \lambda B+\partial_{x} E_{2}=0
$$

with $\rho=-\int f d v$ and $j_{i}=-\int \hat{v}_{i} f d v$. By definition, $\mathscr{A}_{1}^{\lambda} \phi=\mathscr{B}^{\lambda} \psi$ and $\mathscr{A}_{2}^{\lambda} \psi+$ $\left(\mathscr{B}^{\lambda}\right)^{*} \phi=0$, which implies that $\partial_{x} E_{1}=-\partial_{x}^{2} \phi=-\int f d v=\rho$ and $\lambda E_{2}+\partial_{x} B=$ $\partial_{x}^{2} \psi-\lambda^{2} \psi=\int \hat{v}_{2} f d v=-j_{2}$, according the formula (2.12) and the definition of $\mathscr{A}_{i}^{\lambda}, \mathscr{B}^{\lambda}$. We also have $\lambda B+\partial_{x} E_{2}=\lambda \partial_{x} \psi-\lambda \psi_{x}=0$. Thus three of the four equations in (2.14) are true. To check (2.13), we look at (2.12) at the point $(X(t), V(t))$, 
as follows.

$$
\begin{aligned}
f(X(t), V(t)) \\
=-\mu_{e} \phi\left(X(t)-\mu_{p} \psi(X(t)\right. \\
\quad+\mu_{e} \int_{-\infty}^{0} \lambda e^{\lambda s}\left[\phi(X(s ; 0, X(t), V(t)))-\hat{V}_{2}(s ; 0, X(t), V(t)) \psi(X(s ; 0, X(t), V(t)))\right] d s \\
=-\mu_{e} \phi\left(X(t)-\mu_{p} \psi\left(X(t)+\mu_{e} \int_{-\infty}^{0} \lambda e^{\lambda s}\left[\phi(X(t+s))-\hat{V}_{2}(t+s) \psi(X(t+s))\right] d s\right.\right. \\
=-\mu_{e} \phi\left(X(t)-\mu_{p} \psi\left(X(t)+\mu_{e} e^{-\lambda t} \int_{-\infty}^{t}\left(\frac{\partial}{d s}\left(e^{\lambda s}\right) \phi(X(s))-\lambda e^{\lambda s} \hat{V}_{2}(s) \psi(X(s))\right) d s\right.\right. \\
=-\mu_{p} \psi\left(X(t)-\mu_{e} e^{-\lambda t} \int_{-\infty}^{t}\left(e^{\lambda s} \frac{\partial \phi(X(s))}{\partial s}+\lambda e^{\lambda s} \hat{V}_{2}(s) \psi(X(s))\right) d s .\right.
\end{aligned}
$$

Thus

$$
\begin{gathered}
e^{\lambda t} f(X(t), V(t)) \\
=\int_{-\infty}^{t}-\frac{\partial}{\partial s}\left\{e^{\lambda s} \mu_{p} \psi(X(s))\right\}-\mu_{e}\left(e^{\lambda s} \frac{\partial \phi(X(s))}{\partial s}+\lambda e^{\lambda s} \hat{V}_{2}(s) \psi(X(s))\right) d s .
\end{gathered}
$$

Differentiating this expression, we have

$$
\begin{gathered}
\frac{d}{d t}\left\{e^{\lambda t} f(X(t), V(t))\right\} \\
=-\frac{\partial}{\partial t}\left\{e^{\lambda t} \mu_{p} \psi(X(t))\right\}-e^{\lambda t} \mu_{e} \frac{\partial \phi(X(t))}{\partial t}-\lambda e^{\lambda t} \mu_{e} \hat{V}_{2}(t) \psi(X(t)),
\end{gathered}
$$

which is equivalent to (2.13). Finally, we still need to check the equation $\lambda E_{1}=$ $-j_{1}$. Integrating (2.13) in $v$, we get $\lambda \rho+\partial_{x} j_{1}=0$ since all the other terms have vanishing integrals in either $x$ or $v$. Combining it with $\partial_{x} E_{1}=\rho$, we have $\partial_{x}\left(\lambda E_{1}+j_{1}\right)=0$, which implies that $\lambda E_{1}=-j_{1}$ since

$$
\int_{0}^{P} E_{1} d x=-\int_{0}^{P} j_{1} d x=0 .
$$

This completes the proof of Lemma 2.5.

Lemma 2.6. If $\mathscr{L}^{0} \nsupseteq 0$, then there exists $\lambda>0$ such that $\mathscr{L}^{\lambda}$ has a non-trivial nullspace. In particular, if $\mathscr{L}^{0}$ has an even eigenfunction corresponding to a negative eigenvalue, then $\mathscr{L}^{\lambda}$ has a non-trivial even nullspace for some $\lambda>0$.

Proof. We first claim that $\mathscr{L}^{\lambda} \geq 0$ for sufficiently large $\lambda$. Indeed, $\mathscr{A}_{1}^{\lambda} \geq 0$ so that $\left(\mathscr{B}^{\lambda}\right)^{*}\left(\mathscr{A}_{1}^{\lambda}\right)^{-1} \mathscr{B}^{\lambda} \geq 0$. Now let us write $\mathscr{A}_{2}^{\lambda} h=-\partial_{x}^{2} h+\lambda^{2} h-\left(\int \hat{v}_{2} \mu_{p} d v\right) h+\Xi$, where (as in the proof of Lemma 2.4)

$$
\|\Xi\|=\left\|\int \hat{v}_{2} \mu_{e} \int_{-\infty}^{0} \lambda e^{\lambda s} \hat{V}_{2}(s) h(X(s)) d s d v\right\| \leq C\|h\|
$$

with a constant $C$ independent of $\lambda$. Thus there is a constant $C^{\prime}$ such that $\left(\mathscr{A}_{2}^{\lambda} h, h\right) \geq$ $\lambda^{2}(h, h)-C^{\prime}(h, h) \geq 0$ for large $\lambda$. 
Secondly we claim that

$$
\lim _{\lambda \searrow 0} \int_{-\infty}^{0} \lambda e^{\lambda s} m(X(s), V(s)) d s=\mathscr{P}_{m}
$$

in the norm of $L_{\left|\mu_{e}\right|}^{2}$ for all $m \in L_{\left|\mu_{e}\right|}^{2}$. Indeed, letting $M$ denote the spectral measure of the self-adjoint operator $R=-i D$ in the space $L_{\left|\mu_{e}\right|}^{2}$, we have $m(X(s), V(s))=$ $\exp (s D) m=\exp (i s R) m$, so that

$$
\int_{-\infty}^{0} \lambda e^{\lambda s} m(X(s), V(s)) d s=\int_{-\infty}^{0} \lambda e^{\lambda s} \int_{\mathbb{R}} e^{i \alpha s} d M(\alpha) m d s=\int_{\mathbb{R}} \frac{\lambda}{\lambda+i \alpha} d M(\alpha) m .
$$

On the other hand, the projection is $\mathscr{P}=M(\{0\})=\int_{\mathbb{R}} \chi d M$ where $\chi(\alpha)=0$ for $\alpha \neq 0$ and $\chi(1)=0$. Therefore

$$
\left\|\int_{-\infty}^{0} \lambda e^{\lambda s} m(X(s), V(s)) d s-\mathscr{P} m\right\|_{\mathbf{L}_{\left|\mu_{e}\right|}^{2}}^{2}=\int_{\mathbb{R}}\left|\frac{\lambda}{\lambda+i \alpha}-\chi(\alpha)\right|^{2} d\|M(\alpha) m\|_{\mathbf{L}_{\left|\mu_{e}\right|}^{2}}^{2}
$$

by orthogonality of the spectral projections. By the dominated convergence theorem this expression tends to 0 as $\lambda \rightarrow 0$, as we wished to prove.

Thirdly we claim that $\mathscr{L}^{\lambda}-\mathscr{L}^{0} \rightarrow 0$ strongly as $\lambda \rightarrow 0$. Indeed, for all $h \in L_{P}^{2}$,

$$
\mathscr{A}_{2}^{\lambda} h-A_{2}^{0} h=-\int \hat{v}_{2} \mu_{e} \int_{-\infty}^{0} \lambda e^{\lambda s}\left\{\hat{V}_{2}(s) h(X(s))-\mathscr{P}\left(\hat{v}_{2} h\right)\right\} d s d v
$$

so that

$$
\begin{aligned}
\left\|\mathscr{A}_{2}^{\lambda} h-\mathscr{A}_{2}^{0} h\right\|^{2} & \leq C \iint\left[\int_{-\infty}^{0} \lambda e^{\lambda s}\left\{\hat{V}_{2}(s) h(X(s))-\mathscr{P}\left(\hat{v}_{2} h\right)\right\} d s\right]^{2}\left|\mu_{e}\right| d v d x \\
& =C\left\|\int_{-\infty}^{0} \lambda e^{\lambda s}\left\{m(X(s), V(s))-\mathscr{P}_{m}\right\} d s\right\|_{\mathbf{L}_{\left|\mu_{e}\right|}^{2}}^{2}
\end{aligned}
$$

where $m(x, v)=\hat{v}_{2} h(x)$ and $C=\sup _{x} \int\left|\mu_{e}\right| d v$. By the second claim, $\| \mathscr{A}_{2}^{\lambda} h-$ $A_{2}^{0} h \| \rightarrow 0$ as $\lambda \rightarrow 0$. By essentially the same proof, $\mathscr{A}_{1}^{\lambda}$ also tends strongly to $\mathscr{A}_{1}^{0}$ and $\mathscr{B}^{\lambda}$ to $\mathscr{B}^{0}$. It follows that $\mathscr{L}^{\lambda}$ tends strongly to $\mathscr{L}^{0}$. From the hypothesis $\mathscr{L}^{0} \geq 0$, it now follows that $\mathscr{L}^{\lambda} \geq 0$ for sufficiently small $\lambda$.

Fourthly we claim that, for $\sigma>0,\left\|\mathscr{L}^{\lambda} h-\mathscr{L}^{\sigma} h\right\| \rightarrow 0$ as $\lambda \rightarrow \sigma$. Indeed, estimating as in the proof of Lemma 2.4,

$$
\begin{aligned}
\left|\left(\mathscr{A}_{2}^{\lambda} h, k\right)-\left(\mathscr{A}_{2}^{\sigma} h, k\right)\right| & \leq \iiint \mid\left(\lambda e^{\lambda s}-\sigma e^{\sigma s}\right) \mu_{e} h(X(s)|d s d v| k(x) \mid d x \\
& \leq C\|h\|\|k\| \int_{-\infty}^{0}\left|\lambda e^{\lambda s}-\sigma e^{\sigma s}\right| d s \\
& \leq C\|h\|\|k\||\log \lambda-\log \sigma| \rightarrow 0 .
\end{aligned}
$$

Thus $\left\|\mathscr{A}_{2}^{\lambda} h-\mathscr{A}_{2}^{\sigma} h\right\| \rightarrow 0$. Similarly, $\left\|\mathscr{A}_{1}^{\lambda} h-\mathscr{A}_{1}^{\sigma} h\right\|+\left\|\mathscr{B}^{\lambda} h-\mathscr{B}^{\sigma} h\right\| \rightarrow 0$. It follows from Lemma 2.4 that $\left\|\left(\mathscr{A}_{1}^{\lambda}\right)^{-1} h-\left(\mathscr{A}_{1}^{\sigma}\right)^{-1} h\right\| \rightarrow 0$ and hence $\| \mathscr{L}^{\lambda} h-$ $\mathscr{L}^{\sigma} h \| \rightarrow 0$. 
Finally suppose that $\mathscr{L}^{0}$ has a smallest negative eigenvalue $\kappa^{0}$. By the third claim $\mathscr{L}^{\lambda}$ also has a smallest negative eigenvalue $\kappa^{\lambda}$ for $\lambda$ small enough. Because of the self-adjointness,

$$
\kappa^{\lambda}=\inf _{h \in \mathbf{H}_{P}^{2},\|h\|=1}\left(\mathscr{L}^{\lambda} h, h\right)
$$

is the smallest eigenvalue of $\mathscr{L}^{\lambda}$ for every $\lambda$. By the fourth claim $\kappa^{\lambda}$ is a continuous function of $\lambda>0$. But we now know that $\kappa^{\lambda}<0$ for small $\lambda$, while $\kappa^{\lambda}>0$ for large $\lambda$. Therefore $\kappa^{\lambda}=0$ for some $\lambda>0$ and $\mathscr{L}^{\lambda}$ has a nontrivial kernel. If $\mathscr{L}^{0}$ has an even eigenfunction corresponding to a negative eigenvalue, by Lemma 2.4 (iv) we can restrict all operators in the space of even functions. Then repeating above proof, we get a nontrivial even kernel of $\mathscr{L}^{\lambda}$ for some $\lambda>0$. This completes the proof of Lemma 2.6.

Theorem 2.2 on the existence of growing modes follows immediately by combining Lemma 2.5 and Lemma 2.6.

\subsection{Invariants}

In order to prove Theorem 2.1(i) on spectral stability, we will introduce the temporal invariants of the system. First we briefly discuss the well-posedness of the Cauchy problem for the linear system with periodic boundary conditions. Denote by $\mathscr{L}$ the linearized operator corresponding to the linearized Vlasov-Maxwell system (2.6), (2.8) excluding the constraint equation $\partial_{x} E_{1}=-\rho$. That is, $(f(t), E(t), B(t))=$ $e^{t \mathscr{L}}(f(0), E(0), B(0))$ is the solution to the linearized system and the constraint $\partial_{x} E_{1}=-\rho$ is clearly preserved in time. Define

$$
L^{1}=L_{x, v}^{1} \times\left(L_{P}^{1}\right)^{3}, \quad W^{1,1}=W_{x, v}^{1,1} \times\left(W_{P}^{1,1}\right)^{3},
$$

where $W_{P}^{1,1}$ is the space of $P$-periodic functions in $W_{x}^{1,1}$. By Lemma 8 of [25], the semigroup $e^{t \mathscr{L}}$ is well-defined from $L^{1}$ to $L^{1}$. Furthermore, it is also easy to prove that $e^{t \mathscr{L}}$ is well defined from $W^{1,1}$ to $W^{1,1}$.

We consider initial data $(f(0), E(0), B(0)) \in W^{1,1}$ with the set of constraints

$$
\mathscr{C}=\left\{\iint f(0) d v d x=0, \partial_{x} E_{1}(0)=-\int f(0) d v \text { and } \int B(0) d x=0\right\},
$$

which are clearly preserved for all time, for any solution of the linearized system (2.6), (2.8). Since $\int B(t) d x=0$, we can define a periodic magnetic potential $\psi(t)$ such that $B(t)=\partial_{x} \psi(t)$. Now we are ready to define the functional

$$
J\left(f, E_{1}, \psi\right)=\iint \frac{1}{\left|\mu_{e}\right|}\left(f+\mu_{p} \psi\right)^{2} d v d x+\int\left[E_{1}\right]^{2} d x .
$$

In the following discussion we consider initial data $(f(0), E(0), B(0)) \in W^{1,1}$ with the constraint $\mathscr{C}$ and $J(f(0), \psi(0))<\infty$.

Remark. It is important for our discussion that any possible growing mode $\left(e^{\lambda t} f_{g}(x, v), e^{\lambda t} E_{g}, e^{\lambda t} B_{g}\right)$ with $f_{g} \in L_{x, v}^{1}$ and $E_{g}, B_{g} \in L_{P}^{2}$ satisfies these conditions. 
Indeed, by the regularity result mentioned in the proof of Lemma 2.5, we have $\left(f_{g}, E_{g}, B_{g}\right) \in W^{1,1}$. Also $\int e^{\lambda t} B_{g} d x$ and $\iint e^{\lambda t} f_{g} d v d x$ are constant in time, which forces $\iint f_{g} d v d x=\int B_{g}=0$. So $\left(f_{g}, E_{g}, B_{g}\right) \in \mathscr{C}$. So there exists a periodic magnetic potential $\psi_{g}$ such that $B_{g}=\partial_{x} \psi_{g}, E_{g 2}=-\lambda \psi_{g}$. We now check $J\left(f_{g}, \psi_{g}\right)<\infty$. From the linearized Vlasov equation, we have

$$
\lambda f_{g}+D f_{g}=\mu_{e} \hat{v}_{1} E_{g 1}-\mu_{p} \hat{v}_{1} \partial_{x} \psi_{g}-\left(\lambda \mu_{e} \hat{v}_{2}+\lambda \mu_{p}\right) \psi_{g} .
$$

Along the particle trajectory $(X(s), V(s))$, we have

$$
\left\{\hat{V}_{1} \partial_{x} \psi_{g}(X(s))+\lambda \psi_{g}(X(s))\right\} e^{\lambda s}=\frac{\partial}{\partial s}\left\{e^{\lambda s} \psi_{g}(X(s)\} .\right.
$$

On almost every trajectory, $f(X(s), V(s))$ is a bounded function of $s$ so that, upon integration,

$$
f_{g}(x, v)=-\mu_{p} \psi_{g}(x)+\mu_{e} \int_{-\infty}^{0} e^{\lambda s}\left(\hat{V}_{1}(s) E_{g 1}(X(s))-\lambda \hat{V}_{2}(s) \psi_{g}(X(s))\right) d s .
$$

So we can extract the factor $\mu_{e}$ from $f_{g}+\mu_{p} \psi_{g}$, which implies that $J\left(f_{g}, E_{1 g}, \psi_{g}\right)<$ $\infty$.

Here are the invariants.

Lemma 2.7. Consider initial data $(f(0), E(0), B(0)) \in L^{1}$ in the constraint set $\mathscr{C}$ satisfying $J\left(f(0), E_{1}(0), \psi(0)\right)<\infty$. The functional

$$
I\left(f, E_{1}, \psi\right)=J\left(f, E_{1}, \psi\right)-\iint \hat{v}_{2} \mu_{p} \psi^{2} d v d x+\int\left[\left(\partial_{t} \psi\right)^{2}+\left(\partial_{x} \psi\right)^{2}\right] d x
$$

is independent of $t$ for the solution of the linearized system (2.6), (2.8) with such initial data. Furthermore, for all $g \in \operatorname{ker} D$, the functionals

$$
K_{g}(f, \psi)=\iint\left[f+\left(\hat{v}_{2} \mu_{e}+\mu_{p}\right) \psi\right] g d v d x
$$

are also independent of $t$.

Proof. By a standard approximation we may assume that the solution is smooth. We calculate

$$
\begin{aligned}
& \frac{1}{2} \partial_{t} I \\
= & \iint \frac{1}{\left|\mu_{e}\right|}\left(f+\mu_{p} \psi\right)\left(\partial_{t} f+\mu_{p} \partial_{t} \psi\right) d v d x \\
& -\iint \hat{v}_{2} \mu_{p} \psi \partial_{t} \psi d v d x+\frac{1}{2} \int \partial_{t}\left[\left(E_{1}\right)^{2}+\left(\partial_{t} \psi\right)^{2}+\left(\partial_{x} \psi\right)^{2}\right] d x \\
= & \iint \frac{1}{\left|\mu_{e}\right|}\left(f+\mu_{p} \psi\right)\left\{-D f+\mu_{e} \hat{v}_{1} E_{1}-\mu_{p} \hat{v}_{1} \partial_{x} \psi-\mu_{e} \hat{v}_{2} \partial_{t} \psi\right\}-\iint \hat{v}_{2} \mu_{p} \psi \partial_{t} \psi d v d x \\
& +\int\left\{E_{1} \int \hat{v}_{1} f d v+\partial_{t} \psi\left(\partial_{x}^{2} \psi-\int \hat{v}_{2} f d v\right)+\partial_{x} \psi \partial_{x} \partial_{t} \psi\right\} d x
\end{aligned}
$$


by (2.6) and (2.8). In this expression three terms vanish because $D$ is skew and $\hat{v}_{1} \mu_{e}, \hat{v}_{1} \mu_{p}$ and $\hat{v}_{1} \mu_{p}^{2} / \mu_{e}$ are odd functions of $v_{1}$. Furthermore, eight other terms cancel pairwise. We end up with

$$
\frac{1}{2} \partial_{t} I=-\iint \frac{\mu_{p}}{\left|\mu_{e}\right|}\left\{\hat{v}_{1} f \partial_{x} \psi+\psi D f\right\} d v d x=\iint D\left\{\frac{\mu_{p}}{\left|\mu_{e}\right|} f \psi\right\} d v d x=0 .
$$

This proves the invariance of $I$. Next let $g \in \operatorname{ker} D$. Then by (2.6),

$$
\begin{aligned}
\partial_{t} K_{g} & =\iint\left\{\partial_{t} f+\left(\mu_{e} \hat{v}_{2}+\mu_{p}\right) \partial_{t} \psi\right\} g d v d x \\
& =\iint\left\{-D f-\mu_{e} \hat{v}_{1} E_{1}-\mu_{p} \hat{v}_{1} \partial_{x} \psi\right\} g d v d x=0
\end{aligned}
$$

because the first term is $-D(f g)$ while the second and third terms are odd functions of $\hat{v}_{1}$.

Next we get a lower bound for the functional $J$ defined in (2.15).

Lemma 2.8. For any $E_{1}, \psi \in L_{P}^{2}$ and $f \in L_{x, v}^{1}$ with

$$
\iint f d v d x=0, \partial_{x} E_{1}=-\int f d v, J\left(f, E_{1}, \psi\right)<\infty,\left(f+\left(\hat{v}_{2} \mu_{e}+\mu_{p}\right) \psi, g\right)=0
$$

$\forall g \in \operatorname{ker} D$, we have

$$
J\left(f, E_{1}, \psi\right) \geq \iint\left|\mathscr{P}\left(\hat{v}_{2} \psi\right)\right|^{2}\left|\mu_{e}\right| d v d x+\left(\left(\mathscr{B}^{0}\right)^{*}\left(\mathscr{A}_{1}^{0}\right)^{-1} \mathscr{B}^{0} \psi, \psi\right) .
$$

Proof. Our strategy is to fix $\psi$ and to minimize $J$ over all $f$ and $E_{1}$ satisfying the conditions of the lemma. By means of a minimizing sequence, it follows easily that for each $\psi$ there exists a minimum of $J\left(f, E_{1}, \psi\right)$. Call the minimizer $\left(f^{\psi}, E_{1}^{\psi}\right)$. It satisfies

$$
\begin{gathered}
0=\left\langle\frac{\partial J}{\partial f}\left(f^{\psi}, E_{1}^{\psi}, \psi\right), f\right\rangle+\left\langle\frac{\partial J}{\partial E_{1}}\left(f^{\psi}, E_{1}^{\psi}, \psi\right), E_{1}\right\rangle \\
=2 \iint\left(f^{\psi}+\mu_{p} \psi\right) f \frac{d v d x}{\left|\mu_{e}\right|}+2 \int E_{1}^{\psi} E_{1} d x
\end{gathered}
$$

for all test function pairs $\left(f, E_{1}\right)$ in the constraint set $\mathscr{C}$. Let $k^{\psi}=\frac{1}{P} \int_{0}^{P} E_{1}^{\psi} d x$ and $k=\frac{1}{P} \int_{0}^{P} E_{1} d x$. Since $E_{1}^{\psi}-k^{\psi}$ is periodic with zero mean, there is a periodic function $\phi^{\psi}$ such that $E_{1}^{\psi}=k^{\psi}-\partial_{x} \phi^{\psi}$. Then

$$
\begin{gathered}
\int_{0}^{P} E_{1}^{\psi} E_{1} d x=\int_{0}^{P}\left(k^{\psi}-\partial_{x} \phi^{\psi}\right) E_{1} d x \\
=P k^{\psi} k+\int_{0}^{P} \phi^{\psi} \partial_{x} E_{1} d x=P k^{\psi} k-\int_{0}^{P} \int \phi^{\psi} f d v d x .
\end{gathered}
$$

Thus the minimizer satisfies

$$
0=\iint \frac{f^{\psi}+\mu_{p} \psi-\left|\mu_{e}\right| \phi}{\left|\mu_{e}\right|} \frac{f}{\left|\mu_{e}\right|}\left|\mu_{e}\right| d v d x+P k^{\psi} k
$$


We can choose the test function $f$ so that the integral vanishes. Since $k$ is arbitrary, it follows that $k^{\psi}=0$ and hence

$$
\frac{f^{\psi}+\mu_{p} \psi+\mu_{e} \phi^{\psi}}{\mu_{e}} \in(\operatorname{ker} D)^{\perp \perp}=\overline{\operatorname{ker} D}=\operatorname{ker} D .
$$

Writing this result in terms of the projection $\mathscr{P}$ onto $\operatorname{ker} D$, we have $(I-$ $\mathscr{P})\left(\left(f^{\psi}+\mu_{p} \psi+\mu_{e} \phi^{\psi}\right) / \mu_{e}\right)=0$ and the constraint is equivalent to $\mathscr{P}\left(\left(f^{\psi}+\right.\right.$ $\left.\left.\mu_{p} \psi+\hat{v}_{2} \mu_{e} \psi\right) / \mu_{e}\right)=0$. We add to obtain

$$
f^{\psi}+\mu_{p} \psi+\mu_{e} \phi^{\psi}=\mu_{e} \mathscr{P}\left(-\hat{v}_{2} \psi+\phi^{\psi}\right) .
$$

Therefore since $\int\left(\mu_{p}+\mu_{e} \hat{v}_{2}\right) d v=\int \partial \mu / \partial v_{2} d v=0$,

$$
\partial_{x}^{2} \phi^{\psi}=\int f^{\psi} d v=\int\left[f^{\psi}+\left(\mu_{p}+\mu_{e} \hat{v}_{2}\right) \psi\right] d v=\int \mu_{e}(1-\mathscr{P})\left(-\phi^{\psi}+\hat{v}_{2} \psi\right) d v .
$$

Thus

$$
\partial_{x}^{2} \phi^{\psi}+\int \mu_{e} d v \phi^{\psi}-\int \mu_{e} \mathscr{P}\left(\phi^{\psi}\right) d v=-\int \mu_{p} d v \psi-\int \mu_{e} \mathscr{P}\left(\hat{v}_{2} \psi\right) d v,
$$

which means that

$$
-\mathscr{A}_{1}^{0} \phi^{\psi}=-\mathscr{B}^{0} \psi .
$$

The equations (2.20) and (2.21) are satisfied by the minimum. It follows from (2.20) that

$$
\begin{aligned}
J\left(f^{\psi}, \psi\right) & =\iint \frac{1}{\left|\mu_{e}\right|}\left(f^{\psi}+\mu_{p} \psi\right)^{2} d v d x+\int\left(\partial_{x} \phi^{\psi}\right)^{2} d x \\
& =\iint\left|\mu_{e}\right|\left[-\phi^{\psi}+\mathscr{P}\left(-\hat{v}_{2} \psi+\phi^{\psi}\right)\right]^{2} d v d x+\int\left(\partial_{x} \phi^{\psi}\right)^{2} d x \\
& =\iint\left|\mu_{e}\right|\left[(I-\mathscr{P})\left(\phi^{\psi}\right)\right]^{2} d v d x+\iint\left|\mu_{e}\right|\left[\mathscr{P}\left(\hat{v}_{2} \psi\right)\right]^{2} d v d x+\int\left(\partial_{x} \phi^{\psi}\right)^{2} d x
\end{aligned}
$$

by orthogonality. The first term in the last expression can be rewritten as

$$
\int \phi^{\psi}\left\{\int\left|\mu_{e}\right|(I-\mathscr{P})\left(\phi^{\psi}\right) d v\right\} d x=\int \phi^{\psi}\left\{-\int \mu_{e} d v \phi^{\psi}+\int \mu_{e} \mathscr{P}\left(\phi^{\psi}\right) d v\right\} d x .
$$

So from the definition of $\mathscr{A}_{1}^{0}$, the functional $J$ equals

$$
J\left(f^{\psi}, \psi\right)=\iint\left|\mu_{e}\right|\left[\mathscr{P}\left(\hat{v}_{2} \psi\right)\right]^{2} d v d x+\left(\phi^{\psi}, \mathscr{A}_{1}^{0} \phi^{\psi}\right)
$$

at the minimum. By (2.21) the last term equals

$$
\left(\mathscr{B}^{0} \psi,\left(\mathscr{A}_{1}^{0}\right)^{-1} \mathscr{B}^{0} \psi\right)=\left(\left(\mathscr{B}^{0}\right)^{*}\left(\mathscr{A}_{1}^{0}\right)^{-1} \mathscr{B}^{0} \psi, \psi\right) .
$$


Proof of Theorem 2.1 ( $i$ ). To prove spectral stability, suppose there is a growing mode $\left[e^{\lambda t} f, e^{\lambda t} E, e^{\lambda t} B\right]$ with $\operatorname{Re} \lambda>0$. Later we will show that $\lambda$ must be real and positive. We assume this here. We must prove that $\mathscr{L}^{0} \nsucceq 0$. As mentioned in the remark before Lemma 2.7, both the mean of $B$ and the mean of $f$ vanish. We also have $J\left(f, E_{1}, \psi\right)<\infty$ where $\psi$ is the periodic magnetic potential such that $B=\partial_{x} \psi$. By (2.17) and (2.18), we get exponential growth in time unless

$$
I\left(f, E_{1}, \psi\right)=0 \quad \text { and } f+\left(\hat{v}_{2} \mu_{e}+\mu_{p}\right) \psi \in(\operatorname{ker} D)^{\perp} .
$$

Thus Lemma 2.8 is applicable. We deduce from (2.17) and (2.19) that

$$
\begin{aligned}
0= & I(f, \psi) \\
\geq & \left(\left(\mathscr{B}^{0}\right)^{*}\left(\mathscr{A}_{1}^{0}\right)^{-1} \mathscr{B}^{0} \psi, \psi\right) \\
& +\iint\left\{\left|\mu_{e}\right|\left|\mathscr{P}\left(\hat{v}_{2} \psi\right)\right|^{2}-\hat{v}_{2} \mu_{p} \psi^{2}\right\} d v d x+\int\left\{\lambda^{2} \psi^{2}+\left(\partial_{x} \psi\right)^{2}\right\} d x \\
= & \left(\left(\mathscr{B}^{0}\right)^{*}\left(\mathscr{A}_{1}^{0}\right)^{-1} \mathscr{B}^{0} \psi, \psi\right)+\left(\mathscr{A}_{2}^{0} \psi, \psi\right)+\lambda^{2}(\psi, \psi) .
\end{aligned}
$$

Thus

$$
\left(\mathscr{L}^{0} \psi+\lambda^{2} \psi, \psi\right) \leq 0 .
$$

Since $\lambda>0$ and $\psi \neq 0$, it is impossible if $\mathscr{L}^{0} \geq 0$. This proves the spectral stability when $\mathscr{L}^{0} \geq 0$.

\subsection{Splitting Method}

In the following discussion we introduce a new invariant, based on the splitting of $f$ into even and odd parts. It will provide more information on the nature of the instability. First we will use the invariant to prove that every growing mode is real. Secondly, in the unstable case when $\mathscr{L}^{0}$ has a negative eigenvalue, we will get an upper bound of the growth rate which we believe is sharp. These are stated as Theorem 2.1 (ii), (iii).

The derivation of the invariant is based on the splitting of the electron density $f$ into its even and odd parts in the variable $v_{1}$ :

$$
f=f_{e v}+f_{o d}, \quad \text { where } f_{e v}\left(x, v_{1}, v_{2}\right)=\frac{1}{2}\left\{f\left(x, v_{1}, v_{2}\right)+f\left(x,-v_{1}, v_{2}\right)\right\} .
$$

The operator $D$ takes even functions into odd ones, and vice versa. So we can eliminate $f_{e v}$ from the linearized Vlasov equation and thereby obtain a secondorder equation for $f_{o d}$, as follows:

$$
\left(\partial_{t}^{2}-D^{2}\right) f_{o d}=-\mu_{e} D\left(E_{2} \hat{v}_{2}\right)+\left(\int \hat{v}_{1} f_{o d} d v\right) \hat{v}_{1} \mu_{e}
$$

where of course $D$ is given by (2.7). In order to derive (2.22), we equate the even and odd parts of the linearized Vlasov equation (2.6) to obtain

$$
\partial_{t} f_{o d}+D f_{e v}=\left(E_{1}+\hat{v}_{2} B\right) \partial_{v_{1}} f^{0}-\hat{v}_{1} B \partial_{v_{2}} f^{0}
$$

and

$$
\partial_{t} f_{e v}+D f_{o d}=E_{2} \partial_{v_{2}} f^{0} .
$$


Eliminating $f_{e v}$, we find

$$
\begin{aligned}
& \partial_{t}^{2} f_{o d}-D^{2} f_{o d} \\
& =-D\left(E_{2} \partial_{v_{2}} f^{0}\right)+\left(\partial_{t} E_{1}+\hat{v}_{2} \partial_{t} B\right) \partial_{v_{1}} f^{0}-\hat{v}_{1} \partial_{t} B \partial_{v_{2}} f^{0} \\
& =-D\left[E_{2}\left(\hat{v}_{2} \mu_{e}+\mu_{p}\right)\right]+\left(\int \hat{v}_{1} f_{o d} d v-\hat{v}_{2} \partial_{x} E_{2}\right)+\hat{v}_{1} \partial_{x} E_{2}\left(\hat{v}_{2} \mu_{e}+\mu_{p}\right) \\
& =-\mu_{e} D\left(E_{2} \hat{v}_{2}\right)+\left(\int \hat{v}_{1} f_{o d} d v\right) \hat{v}_{1} \mu_{e},
\end{aligned}
$$

which is (2.22). This has the appearance of a second-order wave equation. It is convenient to rewrite it as

$$
\left\{\partial_{t}^{2}-D^{2}\right\}\left(\frac{f_{o d}}{\left|\mu_{e}\right|}\right)=D\left(E_{2} \hat{v}_{2}\right)-\left(\int \hat{v}_{1} f_{o d} d v\right) \hat{v}_{1} .
$$

In the next lemma we derive the energy invariant.

Lemma 2.9. Define the "kinetic" and "potential" energies by

$$
K=\iint \frac{1}{\left|\mu_{e}\right|}\left|\partial_{t} f_{o d}\right|^{2} d v d x+\int\left|\partial_{t} E_{2}\right|^{2} d x
$$

and

$$
\begin{aligned}
W & =\iint \frac{1}{\left|\mu_{e}\right|}\left|D f_{o d}-\mu_{e} \hat{v}_{2} E_{2}\right|^{2} d v d x+\int\left|\partial_{x} E_{2}\right|^{2} d x \\
& +\int\left|\int \hat{v}_{1} f_{o d} d v\right|^{2} d x-\iint \mu_{p} \hat{v}_{2}\left|E_{2}\right|^{2} d v d x .
\end{aligned}
$$

Then $K+W$ is independent of t for any complex-valued solution. Some regularity is required but it is true in particular for any growing mode.

Proof. By approximation we can assume that the solution is as smooth as we wish. We begin with (2.8):

$$
\begin{gathered}
\partial_{x} E_{1}=-\int f d v, \quad \partial_{t} E_{1}=\int \hat{v}_{1} f d v \\
\partial_{t} E_{2}+\partial_{x} B=\int \hat{v}_{2} f d v, \quad \partial_{t} B+\partial_{x} E_{2}=0 .
\end{gathered}
$$

We denote the complex conjugate of $f$ by $f^{*}$. Multiplying (2.25) by $\partial_{t} f_{o d}^{*}$, integrating over $x$ and $v$ and taking the real part, we find

$$
\begin{aligned}
& \frac{1}{2} \frac{d}{d t} \iint \frac{\left|\partial_{t} f_{o d}\right|^{2}+\left|D f_{o d}\right|^{2}}{\left|\mu_{e}\right|} d v d x \\
& =-\operatorname{Re} \iint E_{2} \hat{v}_{2} \partial_{t} D f_{o d}^{*} d v d x-\frac{1}{2} \frac{d}{d t} \int\left|\int \hat{v}_{1} f_{o d} d v\right|^{2} d x .
\end{aligned}
$$


The first term on the right is

$$
\begin{aligned}
& -\operatorname{Re} \iint E_{2} \hat{v}_{2} \partial_{t} D f_{o d}^{*} d v d x \\
& =-\frac{d}{d t} \operatorname{Re} \iint E_{2} \hat{v}_{2} D f_{o d}^{*} d v d x+\operatorname{Re} \iint\left(\partial_{t} E_{2}\right) \hat{v}_{2} D f_{o d}^{*} d v d x .
\end{aligned}
$$

Substituting $D f_{o d}=E_{2} \partial_{v_{2}} f^{0}-\partial_{t} f_{e v}$, we have

$$
\begin{aligned}
& \operatorname{Re} \iint\left(\partial_{t} E_{2}\right) \hat{v}_{2} D f_{o d}^{*} d v d x \\
& =\frac{1}{2} \frac{d}{d t} \int\left|E_{2}\right|^{2} \int \hat{v}_{2} \partial_{v_{2}} f^{0} d v d x-\operatorname{Re} \int \partial_{t} E_{2} \partial_{t}\left(\partial_{t} E_{2}^{*}+\partial_{x} B^{*}\right) d x \\
& =\frac{1}{2} \frac{d}{d t} \int\left|E_{2}\right|^{2}\left(\hat{v}_{2}^{2} \mu_{e}+\mu_{p}\right) d v d x-\frac{1}{2} \frac{d}{d t} \int\left|\partial_{t} E_{2}\right|^{2} d x-\frac{1}{2} \frac{d}{d t} \int\left|\partial_{x} E_{2}\right|^{2} d x
\end{aligned}
$$

by (2.26). Combining these identities, we obtain $d K / d t+d W / d t=0$.

Another important identity is the following.

\section{Lemma 2.10.}

$$
\frac{d^{2}}{d t^{2}}\left\{\frac{1}{2} \iint \frac{1}{\left|\mu_{e}\right|}\left|f_{o d}\right|^{2} d v d x+\frac{1}{2} \int\left|E_{2}\right|^{2} d x\right\}=K-W
$$

for any complex-valued solution. Some regularity is required but it is true in particular for any growing mode.

Proof. We now multiply (2.25) by $f_{o d}^{*}$, integrate and take the real part. The first term on the left gives

$$
\operatorname{Re} \iint \partial_{t}^{2}\left\{\frac{f_{o d}}{\left|\mu_{e}\right|}\right\} f_{o d}^{*} d v d x=\frac{d^{2}}{d t^{2}} \frac{1}{2} \iint \frac{\left|f_{o d}\right|^{2}}{\left|\mu_{e}\right|} d v d x-\iint \frac{\left|\partial_{t} f_{o d}\right|^{2}}{\left|\mu_{e}\right|} d v d x
$$

The first term on the right in (2.25) gives, using (2.24), the real part of

$$
\begin{aligned}
& \iint D\left(E_{2} \hat{v}_{2}\right) f_{o d}^{*} d x d v \\
= & -\iint E_{2} \hat{v}_{2} D f_{o d}^{*}=-2 \iint E_{2} \hat{v}_{2} D f_{o d}^{*}+\iint E_{2} \hat{v}_{2}\left(E_{2}^{*} \partial_{v_{2}} f^{0}-\partial_{t} f_{e v}^{*}\right) \\
= & -2 \iint E_{2} \hat{v}_{2} D f_{o d}^{*}+\iint\left|E_{2}\right|^{2} \hat{v}_{2}\left(\hat{v}_{2} \mu_{e}+\mu_{p}\right) d v d x-\int E_{2} \partial_{t}\left(\int \hat{v}_{2} f_{e v}^{*} d v\right) d x \\
= & -2 \iint E_{2} \hat{v}_{2} D f_{o d}^{*}+\iint\left|E_{2}\right|^{2} \hat{v}_{2}\left(\hat{v}_{2} \mu_{e}+\mu_{p}\right) d v d x-\int E_{2}\left(\partial_{t}^{2}-\partial_{x}^{2}\right) E_{2}^{*} d x,
\end{aligned}
$$


using (2.26) at the last step. Combining all the terms from (2.25) multiplied by $f_{o d}^{*}$, we find

$$
\begin{aligned}
& \frac{d^{2}}{d t^{2}} \frac{1}{2} \iint \frac{\left|f_{o d}\right|^{2}}{\left|\mu_{e}\right|} d v d x \\
= & \iint \frac{\left|\partial_{t} f_{o d}\right|^{2}}{\left|\mu_{e}\right|} d v d x-\iint \frac{\left|D f_{o d}\right|^{2}}{\left|\mu_{e}\right|} d v d x-2 \operatorname{Re} \iint E_{2} \hat{v}_{2} D f_{o d}^{*} d v d x \\
+ & \iint\left|E_{2}\right|^{2} \hat{v}_{2}\left(\hat{v}_{2} \mu_{e}+\mu_{p}\right) d v d x-\operatorname{Re} \int E_{2} \partial_{t}^{2} E_{2}^{*} d x-\int\left|\partial_{x} E_{2}\right|^{2}-\int\left|\int \hat{v}_{1} f_{o d} d v\right|^{2} d x .
\end{aligned}
$$

Finally,

$$
-\operatorname{Re} \int E_{2} \partial_{t}^{2} E_{2}^{*} d x=-\frac{d^{2}}{d t^{2}} \frac{1}{2} \int\left|E_{2}\right|^{2} d x+\int\left|\partial_{t} E_{2}\right|^{2} d x
$$

Combining terms, we deduce Lemma 2.10.

Remark. For any growing mode $\left[e^{\lambda t} f(x, v), e^{\lambda t} E(x), e^{\lambda t} B(x)\right]$ with $\operatorname{Re} \lambda>0$, we show that $(K+W)(0)<\infty$ and thus Lemmas 2.9 and 2.10 are applicable. Indeed, let $\psi(x)$ be the magnetic potential such that $E_{2}=-\lambda \psi$ and $B=\partial_{x} \psi$. By (2.16),

$$
\begin{aligned}
f(x, v) & =-\mu_{p} \psi(x)+\mu_{e} \int_{-\infty}^{0} e^{\lambda s}\left(\hat{V}_{1}(s) E_{1}(X(s))-\lambda \hat{V}_{2}(s) \psi(X(s))\right) d s \\
& =-\mu_{p} \psi(x)+\mu_{e} k(x, v) .
\end{aligned}
$$

So $f_{o d}=\mu_{e} k_{o d}(x, v)$ and $f_{e v}=-\mu_{p} \psi(x)+\mu_{e} k_{e v}(x, v)$. Thus by (2.24),

$$
D f_{o d}=E_{2}\left(\hat{v}_{2} \mu_{e}+\mu_{p}\right)-\lambda f_{e v}=\mu_{e}\left(E_{2} \hat{v}_{2}-\lambda k_{e v}(x, v)\right) \text {. }
$$

Because $E, \psi \in L_{P}^{\infty}, k \in L^{\infty}$ and $\mu_{e} \in L_{x, v}^{1}$, we have $k(x, v) \in L_{\left|\mu_{e}\right|}^{2}$, we have

$$
\iint \frac{1}{\left|\mu_{e}\right|}\left|f_{o d}\right|^{2} d v d x+\iint \frac{1}{\left|\mu_{e}\right|}\left|D f_{o d}-\mu_{e} \hat{v}_{2} E_{2}\right|^{2} d v d x<\infty
$$

Proof of Theorem 2.1 (ii), (iii). Suppose now that there exists a growing mode

$$
\left[e^{\lambda t} f(x, v), e^{\lambda t} E(x), e^{\lambda t} B(x)\right]
$$


with exponent $\lambda=a+i b, a>0$. By the Remark above, Lemmas 2.9 and 2.10 are applicable. By (2.25) we have

(2.30)

$$
\begin{aligned}
& \iint \partial_{t}^{2}\left\{\frac{f_{o d}}{\left|\mu_{e}\right|}\right\} f_{o d}^{*} d v d x+\iint \frac{\left|D f_{o d}\right|^{2}}{\left|\mu_{e}\right|} d v d x \\
& =\iint D\left(E_{2} \hat{v}_{2}\right) f_{o d}^{*} d v d x-\int\left|\int \hat{v}_{1} f_{o d} d v\right|^{2} d x \\
& =-\iint\left|E_{2}\right|^{2} \hat{v}_{2}\left(\hat{v}_{2} \mu_{e}+\mu_{p}\right) d v d x+\int E_{2} \partial_{t}^{2} E_{2}^{*} d x+\int\left|\partial_{x} E_{2}\right|^{2}-\int\left|\int \hat{v}_{1} f_{o d} d v\right|^{2} d x
\end{aligned}
$$

as in (2.27). From (2.30) we have

$$
\begin{aligned}
\lambda^{2} \iint \frac{\left|f_{o d}\right|^{2}}{\left|\mu_{e}\right|} d v d x & =-\iint \frac{\left|D f_{o d}\right|^{2}}{\left|\mu_{e}\right|} d v d x-\iint\left|E_{2}\right|^{2} \hat{v}_{2}\left(\hat{v}_{2} \mu_{e}+\mu_{p}\right) d v d x \\
& +\left(\lambda^{*}\right)^{2} \int\left|E_{2}\right|^{2} d x+\int\left|\partial_{x} E_{2}\right|^{2}+\int\left|\int \hat{v}_{1} f_{o d} d v\right|^{2} d x
\end{aligned}
$$

Taking the imaginary part, we have

$$
2 a b\left(\iint \frac{\left|f_{o d}\right|^{2}}{\left|\mu_{e}\right|} d v d x+\int\left|E_{2}\right|^{2} d x\right)=0,
$$

which implies $b=0$ as desired. This proves part (ii) of Theorem 2.1.

Now we take a growing mode, which is necessarily real. We claim that

$$
W \geq\left(\mathscr{L}^{0} E_{2}, E_{2}\right)
$$

To prove this claim, we apply Lemma 2.8 with $\psi$ replaced by $E_{2}$ and $f$ replaced by $g \equiv D f_{\text {od }}-\left(\hat{v}_{2} \mu_{e}+\mu_{p}\right) E_{2}$. There are several conditions to check. First, $E_{2} \in$ $L_{P}^{2}$. Secondly, $g+\left(\hat{v}_{2} \mu_{e}+\mu_{p}\right) E_{2}=D f_{o d} \in \operatorname{ker} D^{\perp}$. Thirdly, $g$ has zero mean due to the fact that $\hat{v}_{2} \mu_{e}+\mu_{p}$ is the derivative of $\mu$ with respect to $\hat{v}_{2}$. Finally, by (2.29), $J\left(g, E_{2}\right)<\infty$. So, applying Lemma 2.8 , we find that

$$
\begin{aligned}
W & =J\left(g, E_{2}\right)+\int\left|\partial_{x} E_{2}\right|^{2} d x-\iint \hat{v}_{2} \mu_{p}\left|E_{2}\right|^{2} d v d x \\
& \geq\left(\left(\mathscr{B}^{0}\right)^{*}\left(\mathscr{A}_{1}^{0}\right)^{-1} \mathscr{B}^{0} E_{2}, E_{2}\right)+\iint\left|\mathscr{P}\left(\hat{v}_{2} E_{2}\right)\right|^{2}\left|\mu_{e}\right| d v d x \\
& +\int\left|\partial_{x} E_{2}\right|^{2} d x-\iint \hat{v}_{2} \mu_{p}\left|E_{2}\right|^{2} d v d x=\left(\mathscr{L}^{0} E_{2}, E_{2}\right),
\end{aligned}
$$

by definition of $\mathscr{L}^{0}$.

Thus letting

$$
N=\frac{1}{2} \iint \frac{1}{\left|\mu_{e}\right|}\left|f_{o d}\right|^{2} d v d x+\frac{1}{2} \int\left|E_{2}\right|^{2} d x
$$


we have $W \geq\left(\mathscr{L}^{0} E_{2}, E_{2}\right) \geq-\alpha^{2} \int\left|E_{2}\right|^{2} d x \geq-2 \alpha^{2} N$. Hence from Lemma 2.10

$$
\frac{d^{2} N}{d t^{2}}=K-W=K+W-2 W \leq c_{0}+4 \alpha^{2} N
$$

by Lemma 2.9. For a growing mode, we have $N=\exp (2 \lambda t) N(0)$, so that $4 \lambda^{2} \leq$ $4 \alpha^{2}$ or $\lambda \leq \alpha$. This proves part (iii) of Theorem 2.1 and completes the proof of Theorem 2.1.

\section{Linear Stability of Axisymmetric 3D RVM}

In this section, we prove Theorem 1.1. The 3D RVM for a non-neutral electron plasma with external fields is

$$
\begin{gathered}
\partial_{t} f+\hat{v} \cdot \nabla_{x} f-\left(\mathbf{E}+\mathbf{E}^{e x t}+\hat{v} \times\left(\mathbf{B}+\mathbf{B}^{e x t}\right)\right) \cdot \nabla_{v} f=0 \\
\partial_{t} \mathbf{E}-\nabla \times \mathbf{B}=\int \hat{v} f d v=-\mathbf{j} \\
\partial_{t} \mathbf{B}+\nabla \times \mathbf{E}=0 \\
\nabla \cdot \mathbf{E}=-\int f d v=\rho, \quad \nabla \cdot \mathbf{B}=0
\end{gathered}
$$

where $x \in \mathbb{R}^{3}, v \in \mathbb{R}^{3}$. We consider solutions of finite energy. Thus they vanish in some sense as $|x| \rightarrow \infty$.

We use the notation of the introduction. The equilibrium distribution function is assumed to have the form $f^{0}=\mu(e, p)$, with

and equilibrium fields

$$
\begin{gathered}
e=\sqrt{1+|v|^{2}}-\phi^{0}(r, z)-\phi^{e x t}(r, z), \\
p=r\left(v_{\theta}-A_{\theta}^{0}(r, z)-A_{\theta}^{e x t}(r, z)\right)
\end{gathered}
$$

$$
\mathbf{E}^{0}=-\partial_{r} \phi^{0} \vec{e}_{r}-\partial_{z} \phi^{0} \vec{e}_{z}, \mathbf{B}^{0}=-\partial_{z} A_{\theta}^{0} \vec{e}_{r}+\frac{1}{r} \partial_{r}\left(r A_{\theta}^{0}\right) \vec{e}_{z},
$$

with $\left(A_{\theta}^{0}, \phi^{0}\right)$ satisfying the elliptic system (1.2), (1.3). We assume $f^{0}$ to have compact support $S$ in the $(x, v)$ space and $f^{0}, E^{0}, B^{0}$ to be differentiable in the whole space. Such an equilibrium is easily constructed in the appendix by choosing $\phi^{\text {ext }}$, $A_{\theta}^{\text {ext }}$ and $\mu$ properly. We assume that $\mu_{e}<0$ on the set $\{\mu>0\}$.

We will consider axisymmetric perturbations only. Our results and methods in this section are analogous to the $1 \frac{1}{2}$ dimensional case. However, the problem of constructing growing modes in $3 \mathrm{D}$ is more complicated and we do not discuss it in this paper. The solutions now live in the whole space with vanishing boundary conditions at infinity and this will bring in some new technical issues.

We introduce the vector potential $\mathbf{A}=A_{r} \vec{e}_{r}+A_{\theta} \vec{e}_{\theta}+A_{z} \vec{e}_{z}$ and the scalar potential $\phi$ for the perturbation of electromagnetic fields, with $A_{r}, A_{\theta}, A_{z}$ and $\phi$ independent of $\theta$. We impose the Coulomb gauge $\nabla \cdot \mathbf{A}=0$. (It is not necessarily true that $\mathbf{A}$ and $\phi$ vanish at infinity.) The corresponding fields are

$$
\mathbf{E}=-\nabla \phi-\partial_{t} \mathbf{A} \text { and } \mathbf{B}=\nabla \times \mathbf{A}
$$


or

$$
\begin{gathered}
\mathbf{E}=\left(E_{r}, E_{\theta}, E_{z}\right)=\left(-\partial_{r} \phi-\frac{\partial A_{r}}{\partial t},-\frac{\partial A_{\theta}}{\partial t},-\partial_{z} \phi-\frac{\partial A_{z}}{\partial t}\right), \\
\mathbf{B}=\left(B_{r}, B_{\theta}, B_{z}\right)=\left(-\partial_{z} A_{\theta}, \partial_{z} A_{r}-\partial_{r} A_{z}, \frac{1}{r} \partial_{r}\left(r A_{\theta}\right)\right) .
\end{gathered}
$$

Then the linearized Vlasov equation becomes

$$
\partial_{t} f+D f=-\mu_{e} D \phi-\mu_{e} \hat{v} \cdot \partial_{t} \mathbf{A}-r \mu_{p} \partial_{t} A_{\theta}-\mu_{p} D\left(r A_{\theta}\right),
$$

where

$$
D=\hat{v} \cdot \nabla_{x}-\left(\mathbf{E}^{0}+\mathbf{E}^{e x t}+\hat{v} \times\left(\mathbf{B}^{0}+\mathbf{B}^{e x t}\right)\right) \cdot \nabla_{\nu},
$$

and the Maxwell equations become

$$
\Delta \phi=-\rho
$$

together with the vector equation

$$
\frac{\partial^{2}}{\partial t^{2}} \mathbf{A}+\frac{\partial}{\partial t} \nabla \phi-\Delta \mathbf{A}=\mathbf{j}
$$

It is worthwhile to recall that $\Delta=\partial_{z}^{2}+\partial_{r}^{2}+\frac{1}{r} \partial_{r}+\frac{1}{r^{2}} \partial_{\theta}^{2}$, that the $\theta$-component of $\frac{1}{r^{2}} \partial_{\theta}^{2} \mathbf{A}$ is $-\frac{1}{r^{2}} A_{\theta}$ and that $\{\Delta \mathbf{A}\}_{\theta} \neq \Delta\left\{A_{\theta}\right\}$. Let the spaces $L_{S}^{2}, H_{S}^{2}$ consist of the cylindrically symmetric functions (functions of $r$ and $z$ only) in $L^{2}\left(\mathbb{R}^{3}\right), H^{2}\left(\mathbb{R}^{3}\right)$. Let

with the norm

$$
H^{2 \dagger}=\left\{\psi \in H_{S}^{2}\left(\mathbb{R}^{3}\right) \mid \frac{1}{r^{2}} \psi \in L^{2}\left(\mathbb{R}^{3}\right)\right\}
$$

$$
\|\psi\|_{H^{2 \dagger}}=\|\psi\|_{\mathbf{H}_{P}^{2}\left(\mathbb{R}^{3}\right)}^{2}+\left\|\frac{1}{r^{2}} \psi\right\|_{L^{2}}^{2} .
$$

As pointed out to us by Fanghua Lin, for any function $\psi(r, z)$ we have

$$
-\Delta\left(\psi e^{i \theta}\right)=\left(-\partial_{z z} \psi-\partial_{r r} \psi-\frac{1}{r} \partial_{r} \psi+\frac{1}{r^{2}} \psi\right) e^{i \theta} .
$$

Furthermore, $\psi(r, z) \in H^{2 \dagger}\left(\mathbb{R}^{3}\right)$ is equivalent to $\psi e^{i \theta} \in H^{2}\left(\mathbb{R}^{3}\right)$, because $\psi e^{i \theta} \in$ $H^{2}$ implies that $\left(\frac{1}{r} \partial_{\theta}\right)^{2}\left(\psi e^{i \theta}\right) \in L^{2}$, which in turn implies $\frac{1}{r^{2}} \psi \in L^{2}$. Thus the singular factor $1 / r^{2}$ is artificial, introduced merely by the change of coordinates. We can apply the usual elliptic regularity theorem to the operator $-\partial_{z z}-\partial_{r r}-$ $\frac{1}{r} \partial_{r}+\frac{1}{r^{2}}$. This is important when we consider the regularity of the steady states (in the appendix) and it will allow us to prove the continuity of steady fields in the whole space. Recall that the 0 -operators $\mathscr{A}_{i}^{0}, \mathscr{B}^{0}, \mathscr{L}^{0}$ are defined by (1.4), (1.5), (1.6) and (1.7). We shall prove that these operators are well-defined.

By a growing mode we mean a solution of the linearized axisymmetric system of the form $\left(e^{\lambda t} f_{g}(x, v), e^{\lambda t} \mathbf{E}_{g}(x), e^{\lambda t} \mathbf{B}_{g}(x)\right)$ with $\operatorname{Re} \lambda>0$ where $\mathbf{E}_{g}, \mathbf{B}_{g} \in L_{S}^{2}$ and $f_{g} \in L_{x v}^{1} \cap L_{x v}^{\infty}$.

In analogy with the $1 \frac{1}{2}$ dimensional case, we also introduce the following operators depending on a positive parameter $\lambda$. Even though we do not use these 
$\lambda$-operators to find growing modes in the 3D case, it is still worthwhile to study them. On the one hand, they are more explicit than the 0 -operators which are their limits. On the other hand, the $\lambda$-operators will still be useful in the future search for growing modes, as we can see from the $1 \frac{1}{2} \mathrm{D}$ case. First we introduce the particle paths $(X(t ; x, v), V(t ; x, v))$, which are the characteristics of $D$. They are defined as the solutions of the ODE

$$
\dot{X}=\hat{V}, \quad \dot{V}=-\left(\mathbf{E}^{0}+\mathbf{E}^{e x t}\right)(X)-\hat{V} \times\left(\mathbf{B}^{0}+\mathbf{B}^{e x t}\right)(X)
$$

with initial conditions $X(0)=x, V(0)=v$. Let $\lambda>0$ and define

$$
\begin{gathered}
\mathscr{A}_{1}^{\lambda} h=-\partial_{z z} h-\partial_{r r} h-\frac{1}{r} \partial_{r} h-\int \mu_{e} d v h+\int \mu_{e} \int_{-\infty}^{0} \lambda e^{\lambda s} h(X(s)) d s d v, \\
\mathscr{A}_{2}^{\lambda}(h)=-\partial_{z z} h-\partial_{r r} h-\frac{1}{r} \partial_{r} h+\frac{1}{r^{2}} h+\lambda^{2} h-r h \int \hat{v}_{\theta} \mu_{p} d v \\
-\int \hat{v}_{\theta} \mu_{e} \int_{-\infty}^{0} \lambda e^{\lambda s} \hat{V}_{\theta}(s) h(X(s)) d s d v \\
\mathscr{B}^{\lambda}(h)=\int \mu_{e} \int_{-\infty}^{0} \lambda e^{\lambda s} \hat{V}_{\theta}(s) h(X(s)) d s d v-h \int \hat{v}_{\theta} \mu_{e} d v
\end{gathered}
$$

and

$$
\mathscr{L}^{\lambda}=\left(\mathscr{B}^{\lambda}\right)^{*}\left(\mathscr{A}_{1}^{\lambda}\right)^{-1} \mathscr{B}^{\lambda}+A_{2}^{\lambda},
$$

which will be shown to be well-defined. First we discuss the basic properties of these $\lambda$-operators and 0 -operators.

Lemma 3.1. (i) The operators $\mathscr{A}_{1}^{\lambda}, \mathscr{A}_{1}^{0}$ are self-adjoint operators on $L_{S}^{2}$ with the common domain $H_{S}^{2}$. The operators $\mathscr{A}_{2}^{\lambda}, \mathscr{A}_{2}^{0}, \mathscr{L}^{\lambda}$ and $\mathscr{L}^{0}: H^{2 \dagger} \rightarrow L_{S}^{2}$ are selfadjoint .

(ii) $\mathscr{A}_{1}^{\lambda}>0$ for all $\lambda \geq 0$.

(iii) The essential spectrum of each of the operators $\mathscr{A}_{1}^{\lambda}, \mathscr{A}_{1}^{0}, \mathscr{A}_{2}^{0}, \mathscr{L}^{0}$ is $[0, \infty)$ and the essential spectrum of both of the operators $\mathscr{A}_{2}^{\lambda}, \mathscr{L}^{\lambda}$ is $\left[\lambda^{2}, \infty\right)$.

(iv) $\mathscr{L}^{\lambda} \rightarrow \mathscr{L}^{0}$ strongly in $L_{S}^{2}$.

Proof. First we show that the operators $\mathscr{A}_{i}^{\lambda}, \mathscr{A}_{i}^{0}, \mathscr{L}^{\lambda}$ and $\mathscr{L}^{0}$ map a cylindrically symmetric function to another cylindrically symmetric function. It can be checked directly that the particle trajectory (3.5) has the property

$$
(X(t ; R x, R v), V(t ; R x, R v))=(R X(t ; x, v), R V(t ; x, v))
$$


for any matrix $R$ representing a rotation with respect to the $z$-axis. So

$$
\begin{aligned}
\int \mu_{e} \int_{-\infty}^{0} \lambda e^{\lambda s} \phi(X(s ; R x, v)) d s d v & =\int \mu_{e} \int_{-\infty}^{0} \lambda e^{\lambda s} \phi(X(s ; R x, R v)) d s d v \\
& =\int \mu_{e} \int_{-\infty}^{0} \lambda e^{\lambda s} \phi(R X(s ; x, v)) d s d v \\
& =\int \mu_{e} \int_{-\infty}^{0} \lambda e^{\lambda s} \phi(X(s ; x, v)) d s d v
\end{aligned}
$$

and thus $\mathscr{A}_{1}^{\lambda}$ is symmetry preserving. Similarly $\mathscr{A}_{2}^{\lambda}, \mathscr{B}^{\lambda}, \mathscr{L}^{\lambda}$ can be proved to be symmetry preserving. As in the proof of Lemma $2.6, \mathscr{A}_{1}^{\lambda}, \mathscr{A}_{2}^{\lambda}, \mathscr{B}^{\lambda}$ converge to $\mathscr{A}_{1}^{0}, \mathscr{A}_{2}^{0}, \mathscr{B}^{0}$ strongly, so $\mathscr{A}_{1}^{0}, \mathscr{A}_{2}^{0}, \mathscr{B}^{0}$ and $\mathscr{L}^{0}$ are also symmetry preserving.

The proofs of self-adjointness of these operators and the positivity of the operators $\mathscr{A}_{1}^{\lambda}, \mathscr{A}_{1}^{0}$ are essentially the same as in the $1 \frac{1}{2} \mathrm{D}$ case, so we skip them here. It is also easy to see that $\mathscr{B}^{\lambda}$ and $\mathscr{B}^{0}$ are bounded operators on $L_{S}^{2}$. We concentrate on part (iii) of the lemma, dealing with the essential spectra of the operators.

The spectra are entirely different from the periodic $1 \frac{1}{2} \mathrm{D}$ case because of the unboundedness of the spatial variables. To find their essential spectra, we shall use Weyl's theorem. Define

$$
K^{\lambda} \phi=\int \mu_{e} d v \phi-\int \mu_{e} \int_{-\infty}^{0} \lambda e^{\lambda s} \phi(X(s)) d s d v .
$$

Note that $\mathscr{A}_{1}^{\lambda}=-\Delta-\mathscr{K}^{\lambda}$ and $K^{\lambda} \leq 0$, as in the proof of Lemma 2.4.

We claim that $K^{\lambda}$ is relatively compact with respect to $-\Delta$. For the proof in case $\lambda>0$, we notice that $K^{\lambda} \phi=\int f d v$ where $f=\mu_{e} \phi-\mu_{e} \int_{-\infty}^{0} \lambda e^{\lambda s} \phi(X(s)) d s$ satisfies the equation

$$
\lambda f+\left(\hat{v} \cdot \nabla_{x}-\left(\mathbf{E}^{0}+\mathbf{E}^{e x t}+\hat{v} \times\left(\mathbf{B}^{0}+\mathbf{B}^{e x t}\right)\right) \cdot \nabla_{v}\right) f=\mu_{e} \hat{v} \cdot \nabla_{x} \phi,
$$

which can be seen by considering (3.6) along a trajectory, multiplying by $\exp (\lambda s)$ and integrating. So

$$
\hat{v} \cdot \nabla_{x} f=\mu_{e} \hat{v} \cdot \nabla_{x} \phi-\lambda f+\nabla_{v} \cdot\left(\left(\mathbf{E}^{0}+\mathbf{E}^{e x t}+\hat{v} \times\left(\mathbf{B}^{0}+\mathbf{B}^{e x t}\right)\right) f\right) .
$$

As in the proof of Lemma 2.4, we have

$$
\|f\|_{L_{x v}^{2}} \leq\left(\int \sup _{x}\left|\mu_{e}\right| d v\right)\|\phi\|_{L^{2}} .
$$

and also

$$
\left\|\left(\mathbf{E}^{0}+\mathbf{E}^{e x t}+\hat{v} \times\left(\mathbf{B}^{0}+\mathbf{B}^{e x t}\right)\right) f\right\|_{L_{x v}^{2}}+\left\|\mu_{e} \hat{v} \cdot \nabla_{x} \phi\right\|_{L_{x v}^{2}} \leq C\|\phi\|_{H^{1}} .
$$

Thus by (3.7) we have

$$
\left\|\hat{v} \cdot \nabla_{x} f\right\|_{L_{x v}^{2}} \leq C\|\phi\|_{H^{1}}
$$


Since $\mu_{e}$ is smooth and has compact support in $x, v$ (so that $f$ also has compact support) and since $\partial \hat{v}$ is bounded, equation (3.7) and the estimates above imply that

$$
\left\|K^{\lambda} \phi\right\|_{H^{\frac{1}{4}\left(\mathbb{R}_{x}^{3}\right)}} \leq C\|\phi\|_{H^{1}\left(\mathbb{R}_{x}^{3}\right)}
$$

for some constant $C$, by the averaging lemma in [5]. Since $K^{\lambda} \phi$ has compact support, estimate (3.8) implies that $K^{\lambda}$ maps a bounded set in $H^{1}\left(\mathbb{R}_{x}^{3}\right)$ into a compact set in $L^{2}\left(\mathbb{R}_{x}^{3}\right)$. Thus $K^{\lambda}$ is relatively compact with respect to $-\Delta$. It follows from Weyl's Theorem that $\sigma_{\text {ess }}\left(\mathscr{A}_{1}^{\lambda}\right)=\sigma_{\text {ess }}\left(-\Delta-K^{\lambda}\right)=[0, \infty)$. The operator $\mathscr{A}_{1}^{0}$ can be written as $-\Delta-K^{0}$ where $K^{0} \phi=\int f d v$ with $f=\mu_{e} \phi-\mathscr{P}\left(\mu_{e} \phi\right)$. By the same reasoning, $K^{0}$ is relatively compact with respect to $-\Delta$ and $\sigma_{e s s}\left(\mathscr{A}_{1}^{0}\right)=[0, \infty)$.

Now define the unitary operator $U: L^{2}\left(\mathbb{R}^{3}\right) \rightarrow L^{2}\left(\mathbb{R}^{3}\right)$ by $U \phi=\phi e^{i \theta}$. By (3.4), the operator $(-\Delta)_{m a g}:=-\partial_{z z}-\partial_{r r}-\frac{1}{r} \partial_{r}+\frac{1}{r^{2}}$, which is defined on the space $H^{2 \dagger}$, can be written as $(-\Delta)_{\text {mag }}=U^{*}(-\Delta) U$. Thus $(-\Delta)_{\text {mag }}$ is self-adjoint with essential spectrum $[0, \infty)$. We will show that for any $\lambda \geq 0, \mathscr{L}^{\lambda}$ is a relatively compact perturbation of $(-\Delta)_{m a g}+\lambda^{2}$. For $\lambda>0$, we write

$$
\mathscr{L}^{\lambda}=(-\Delta)_{m a g}+\lambda^{2}+G^{\lambda} .
$$

For $A_{\theta} \in H^{2 \dagger}$, we have $G^{\lambda} A_{\theta}=\vec{e}_{\theta} \cdot \int \hat{v} f d v$, where $f$ is defined by

$$
f=-\mu_{e} \phi+\mu_{e} \int_{-\infty}^{0} \lambda e^{\lambda s} \phi(X(s)) d s-\mu_{e} \int_{-\infty}^{0} \lambda e^{\lambda s} \hat{V}_{\theta}(s) A_{\theta}(X(s)) d s+\mu_{p} r A_{\theta} .
$$

with $\phi$ solving

$$
\begin{aligned}
& -\Delta \phi-\int \mu_{e} d v \phi+\int \mu_{e} \int_{-\infty}^{0} \lambda e^{\lambda s} \phi(X(s)) d s d v \\
& =\int \mu_{e} \int_{-\infty}^{0} \lambda e^{\lambda s} \hat{V}_{\theta}(s) A_{\theta}(X(s)) d s d v+r A_{\theta} \int \mu_{p} d v .
\end{aligned}
$$

This follows from the definition of $\lambda$-operators by simple calculation. We will show that $G^{\lambda}$ is relatively compact with respect to $-\Delta_{m a g}$. Define the electromagnetic fields that correspond to $A_{\theta}$ and $\phi$ (with $A_{r}=A_{z}=0$ ) by

$$
\begin{gathered}
\mathbf{E}=\left(E_{r}, E_{\theta}, E_{z}\right)=\left(-\partial_{r} \phi,-\lambda A_{\theta},-\partial_{z} \phi\right) \\
\mathbf{B}=\left(B_{r}, B_{\theta}, B_{z}\right)=\left(-\partial_{z} A_{\theta}, 0, \frac{1}{r} \partial_{r}\left(r A_{\theta}\right)\right) .
\end{gathered}
$$

Then the equation (3.9) satisfied by $f$ can be written as

$$
\begin{aligned}
& \lambda f+\left(\hat{v} \cdot \nabla_{x}-\left(\mathbf{E}^{0}+\mathbf{E}^{e x t}+\hat{v} \times\left(\mathbf{B}^{0}+\mathbf{B}^{e x t}\right)\right) \cdot \nabla_{v}\right) f \\
& =(\mathbf{E}+\hat{v} \times \mathbf{B}) \cdot \nabla_{v} f^{0}
\end{aligned}
$$


which comes directly from the original linearized Vlasov equation. Now $\mathscr{A}_{1}^{\lambda} \geq$ $-\Delta$, as in the proof of Lemma 2.4(ii). By (3.10) and the Hölder inequality we have

$$
\|\nabla \phi\|_{2}^{2} \leq\left(\mathscr{A}_{1}^{\lambda} \phi, \phi\right)=\left(\mathscr{B}^{\lambda} A_{\theta}, \phi\right) \leq C\left\|A_{\theta}\right\|_{2}\|\nabla \phi\|_{2} .
$$

The last inequality is true because

$$
\left(\mathscr{B}^{\lambda} A_{\theta}, \phi\right)=\iint \mu_{e} \int_{-\infty}^{0} \lambda e^{\lambda s} \hat{V}_{\theta}(s) A_{\theta}(X(s)) d s d v \phi d x-\iint \hat{v}_{\theta} \mu_{e} d v A_{\theta} \phi d x .
$$

The last integral is bounded by $\left\|A_{\theta}\right\|_{2}\|\phi\|_{6}\left\|\int \hat{v}_{\theta} \mu_{e} d v\right\|_{3}$. The first integral is bounded by

$$
\begin{aligned}
& \int_{0}^{\infty} \lambda e^{\lambda s}\left[\iint\left|\mu_{e}\right|\left|A_{\theta}(X)\right|^{2} d V d X\right]^{1 / 2}\left[\iint\left|\mu_{e} \phi^{6}\right| d v d x\right]^{1 / 6}\left[\iint\left|\mu_{e}\right| d v d x\right]^{1 / 3} d s \\
& \leq\left(\sup _{x} \int\left|\mu_{e}\right| d v\right)\left\|A_{\theta}\right\|_{2}\|\phi\|_{6} \leq C\left\|A_{\theta}\right\|_{2}\|\nabla \phi\|_{2} .
\end{aligned}
$$

So $\|\nabla \phi\|_{2} \leq C\left\|A_{\theta}\right\|_{2}$ and thus $\|\mathbf{E}\|_{2} \leq(C+\lambda)\left\|A_{\theta}\right\|_{2}$. By the definition of $H^{2 \dagger}$, we have $\|\mathbf{B}\|_{2} \leq\left\|A_{\theta}\right\|_{H^{2 \dagger}}$. From (3.11) we have

$$
\hat{v} \cdot \nabla_{x} f=-\lambda f+\nabla_{v} \cdot\left\{\left(\mathbf{E}^{0}+\mathbf{E}^{e x t}+\hat{v} \times\left(\mathbf{B}^{0}+\mathbf{B}^{e x t}\right)\right) f+(\mathbf{E}+\hat{v} \times \mathbf{B}) f^{0}\right\} .
$$

So for $i=1,2,3$ we have

$$
\begin{aligned}
\hat{v} \cdot \nabla_{x}\left(\hat{v}_{i} f\right) & =-\lambda \hat{v}_{i} f+\nabla_{v} \cdot\left\{\left(\mathbf{E}^{0}+\mathbf{E}^{e x t}+\hat{v} \times\left(\mathbf{B}^{0}+\mathbf{B}^{\text {ext }}\right)\right) \hat{v}_{i} f+(\mathbf{E}+\hat{v} \times \mathbf{B}) \hat{v}_{i} f^{0}\right\} \\
& -\nabla_{v}\left(\hat{v}_{i}\right) \cdot\left\{\left(\mathbf{E}^{0}+\mathbf{E}^{e x t}+\hat{v} \times\left(\mathbf{B}^{0}+\mathbf{B}^{\text {ext }}\right)\right) f+(\mathbf{E}+\hat{v} \times \mathbf{B}) f^{0}\right\} .
\end{aligned}
$$

Integrating (3.11) along the trajectory, we have

$$
f=\int_{-\infty}^{0} e^{\lambda s}\left((\mathbf{E}+\hat{v} \times \mathbf{B}) \cdot \nabla_{v} f^{0}\right)(X(s ; x, v), V(s ; x, v)) d s
$$

whence $\|f\|_{L_{x v}^{2}} \leq \frac{C}{\lambda}\left(\|\mathbf{E}\|_{2}+\|\mathbf{B}\|_{2}\right) \leq C(\lambda)\left\|A_{\theta}\right\|_{H^{2 \dagger}}$. Since

$$
\begin{aligned}
& \left\|\left(\mathbf{E}^{0}+\mathbf{E}^{e x t}+\hat{v} \times\left(\mathbf{B}^{0}+\mathbf{B}^{\text {ext }}\right)\right) f+(\mathbf{E}+\hat{v} \times \mathbf{B}) f^{0}\right\|_{2} \\
& \leq C\left(\|\mathbf{E}\|_{2}+\|\mathbf{B}\|_{2}\right) \leq C(\lambda)\left\|A_{\theta}\right\|_{H^{2 \dagger}}
\end{aligned}
$$

and $\left|\nabla_{v}(\hat{v})\right| \leq C$, we have

$$
\left\|\int \hat{v}_{i} f d v\right\|_{H^{1 / 4}} \leq C(\lambda)\left\|A_{\theta}\right\|_{H^{2 \dagger}} \quad(i=1,2,3)
$$

by (3.12) and the averaging lemma. Thus for any fixed bounded sequence $\left\{A_{\theta}^{n}\right\}$ in $H^{2 \dagger}$, there exists a convergent subsequence of $j^{n}$ in $L^{2}\left(\mathbb{R}_{x}^{3}\right)$ where $j^{n}=\int \hat{v} f^{n} d v$ with $f^{n}$ defined by (3.9) and (3.10) from $A_{\theta}^{n}$. This implies the existence of a convergent subsequence of $G^{\lambda} A_{\theta}^{n}=\vec{e}_{\theta} \cdot \int \hat{v} f^{n} d v$ in $L^{2}\left(\mathbb{R}_{x}^{3}\right)$ and thus $G^{\lambda}$ is relatively compact to $(-\Delta)_{\text {mag }}$ and the essential spectrum of $\mathscr{L}^{\lambda}$ is $\left[\lambda^{2}, \infty\right)$ by Weyl's theorem. 
We use the same strategy to deal with the operator $\mathscr{L}^{0}$. We write $\mathscr{L}^{0}=$ $(-\Delta)_{\text {mag }}+G^{0}$. Using the previous definitions, for $A_{\theta} \in H^{2 \dagger}$, we can write $G^{0} A_{\theta}=$ $\vec{e}_{\theta} \cdot \int \hat{v} f d v$, where $f$ is defined by

$$
f=-\mu_{e} \phi+\mu_{e} \mathscr{P}(\phi)-\mu_{e} \mathscr{P}\left(\hat{v}_{\theta} A_{\theta}\right)-\mu_{p} r A_{\theta}
$$

with $\phi$ satisfying

$$
-\Delta \phi-\int \mu_{e} d v \phi+\int \mathscr{P}\left(\mu_{e} \phi\right) d v=\int \mathscr{P}\left(\mu_{e} \hat{v}_{\theta} A_{\theta}\right) d v+\int \mu_{p} d v r A_{\theta} .
$$

We have $D f=-\mu_{e} D \phi-\mu_{p} D\left(r A_{\theta}\right)$ which can be written as

$$
\hat{v} \cdot \nabla_{x} f=\nabla_{v} \cdot\left(\left(\mathbf{E}^{0}+\mathbf{E}^{e x t}+\hat{v} \times\left(\mathbf{B}^{0}+\mathbf{B}^{e x t}\right)\right) f+(\mathbf{E}+\hat{v} \times \mathbf{B}) f^{0}\right)
$$

with $\mathbf{E}$ and $\mathbf{B}$ defined by

$$
\mathbf{E}=\left(-\partial_{r} \phi, 0,-\partial_{z} \phi\right), \mathbf{B}=\left(-\partial_{z} A_{\theta}, 0, \frac{1}{r} \partial_{r}\left(r A_{\theta}\right)\right) .
$$

Similarly, from (3.14) we have $\|\phi\|_{6} \leq\|\mathbf{E}\|_{2} \leq C\left\|A_{\theta}\right\|_{2}$. Then from (3.13) we have $\|f\|_{L_{x v}^{2}} \leq C\left(\|\phi\|_{6}+\left\|A_{\theta}\right\|_{2}\right) \leq C^{\prime}\left\|A_{\theta}\right\|_{2}$. By the same argument as we used to prove the relative compactness of $G^{\lambda}$, we deduce that $G^{0}$ too is relatively compact with respect to $(-\Delta)_{\text {mag }}$. Thus the essential spectrum of $\mathscr{L}^{0}$ is $[0, \infty)$.

Now we prove (iv), that $\mathscr{L}^{\lambda} \rightarrow \mathscr{L}^{0}$ strongly as $\lambda \rightarrow 0+$. Compared with the $1 \frac{1}{2} \mathrm{D}$ case, the main difficulty here is that $\left(\mathscr{A}_{1}^{\lambda}\right)^{-1}$ and $\left(\mathscr{A}_{1}^{0}\right)^{-1}$ are unbounded operators. Given $A_{\theta} \in H^{2 \dagger}$, we define $\phi^{\lambda}$ and $\phi^{(0)}$ by

$$
\mathscr{A}_{1}^{\lambda} \phi^{\lambda}=\left(-\Delta-K^{\lambda}\right) \phi^{\lambda}=\mathscr{B}^{\lambda} A_{\theta}
$$

and

$$
\mathscr{A}_{1}^{0} \phi^{(0)}=\left(-\Delta-K^{0}\right) \phi^{(0)}=\mathscr{B}^{0} A_{\theta} .
$$

We will show that $\left\|\phi^{\lambda}-\phi^{(0)}\right\|_{6} \rightarrow 0$. We have

$$
\mathscr{A}_{1}^{\lambda}\left(\phi^{\lambda}-\phi^{(0)}\right)=\mathscr{B}^{\lambda} A_{\theta}-\mathscr{B}^{0} A_{\theta}+\left(K^{\lambda}-K^{0}\right) \phi^{(0)} .
$$

Multiplying this expression by $\phi^{\lambda}-\phi^{(0)}$ and integrating, we have

$$
\begin{aligned}
\left\|\nabla\left(\phi^{\lambda}-\phi^{(0)}\right)\right\|_{2}^{2} & \leq\left(\mathscr{B}^{\lambda} A_{\theta}-\mathscr{B}^{0} A_{\theta}, \phi^{\lambda}-\phi^{(0)}\right)+\left(\left(K^{\lambda}-K^{0}\right) \phi^{(0)}, \phi^{\lambda}-\phi^{(0)}\right) \\
& =I+I I
\end{aligned}
$$

since $K^{\lambda} \leq 0$. As in the proof of Lemma 2.6, we have

$$
\int_{-\infty}^{0} \lambda e^{\lambda s} m(X(s), V(s)) d s \rightarrow \mathscr{P}_{m} \text { in } L_{\left|\mu_{e}\right|}^{2} \text {, as } \lambda \rightarrow 0+
$$


for any $m \in L_{\left|\mu_{e}\right|}^{2}$. So

$$
\begin{aligned}
I & =\iint \mu_{e}\left(\int_{-\infty}^{0} \lambda e^{\lambda s} \hat{V}_{\theta}(s) A_{\theta}(X(s)) d s-\mathscr{P}\left(\hat{v}_{\theta} A_{\theta}\right)\right)\left(\phi^{\lambda}-\phi^{(0)}\right) d v d x \\
& \leq\left\|\left|\mu_{e}\right|^{\frac{1}{6}}\left(\phi^{\lambda}-\phi^{(0)}\right)\right\|_{L_{x v}^{6}}\left\|\left|\mu_{e}\right|^{\frac{1}{3}}\right\|_{L_{x v}^{3}}\left\|\int_{-\infty}^{0} \lambda e^{\lambda s} \hat{V}_{\theta}(s) A_{\theta}(X(s)) d s-\mathscr{P}\left(\hat{v}_{\theta} A_{\theta}\right)\right\|_{L_{\left|\mu_{e}\right|}^{2}} \\
& \leq \omega_{1}(\lambda)\left\|\phi^{\lambda}-\phi^{(0)}\right\|_{6}
\end{aligned}
$$

where $\omega_{1}(\lambda) \rightarrow 0$ as $\lambda \rightarrow 0+$. As in the proof of Lemma 3.1, we have $\left\|\phi^{(0)}\right\|_{6} \leq$ $C\left\|A_{\theta}\right\|_{2}$. Then by the same estimate as above, $I I \leq \omega_{2}(\lambda)\left\|\phi^{\lambda}-\phi^{(0)}\right\|_{6}$ with $\omega_{2}(\lambda) \rightarrow 0$ as $\lambda \rightarrow 0+$. So

$$
\begin{aligned}
\left\|\nabla\left(\phi^{\lambda}-\phi^{(0)}\right)\right\|_{2}^{2} & \leq\left(\omega_{1}(\lambda)+\omega_{2}(\lambda)\right)\left\|\phi^{\lambda}-\phi^{(0)}\right\|_{6} \\
& \leq C\left(\omega_{1}(\lambda)+\omega_{2}(\lambda)\right)\left\|\nabla\left(\phi^{\lambda}-\phi^{(0)}\right)\right\|_{2}
\end{aligned}
$$

which implies that $\left\|\nabla\left(\phi^{\lambda}-\phi^{(0)}\right)\right\|_{2} \rightarrow 0$ and $\left\|\phi^{\lambda}-\phi^{(0)}\right\|_{6} \rightarrow 0$ as $\lambda \rightarrow 0+$.

By definition, $\mathscr{L}^{\lambda} A_{\theta}=\left(\mathscr{B}^{\lambda}\right)^{*} \phi^{\lambda}+\mathscr{A}_{2}^{\lambda} A_{\theta}$ and $\mathscr{L}^{0} A_{\theta}=\left(\mathscr{B}^{0}\right)^{*} \phi^{(0)}+\mathscr{A}_{2}^{0} A_{\theta}$. Using (3.15), we have $\left\|\mathscr{A}_{2}^{\lambda} A_{\theta}-\mathscr{A}_{2}^{0} A_{\theta}\right\|_{2} \rightarrow 0$ as $\lambda \rightarrow 0+$. By the same proof as above,

$$
\begin{aligned}
\left\|\left(\mathscr{B}^{\lambda}\right)^{*} \phi^{\lambda}-\left(\mathscr{B}^{0}\right)^{*} \phi^{(0)}\right\|_{2} & \leq\left\|\left(\mathscr{B}^{\lambda}\right)^{*}\left(\phi^{\lambda}-\phi^{(0)}\right)\right\|_{2}+\left\|\left(\left(\mathscr{B}^{\lambda}\right)^{*}-\left(\mathscr{B}^{0}\right)^{*}\right) \phi^{(0)}\right\|_{2} \\
& \leq C\left\|\phi^{\lambda}-\phi^{(0)}\right\|_{6}+\omega_{3}(\lambda)\left\|\phi^{(0)}\right\|_{6}
\end{aligned}
$$

for some $\omega_{1}(\lambda) \rightarrow 0$ as $\lambda \rightarrow 0+$. Thus $\left\|\left(\mathscr{B}^{\lambda}\right)^{*} \phi^{\lambda}-\left(\mathscr{B}^{0}\right)^{*} \phi^{(0)}\right\|_{2} \rightarrow 0$ and

$$
\left\|\mathscr{L}^{\lambda} A_{\theta}-\mathscr{L}^{0} A_{\theta}\right\|_{2} \rightarrow 0
$$

as we wanted to prove.

Lemma 3.2. For any growing mode, $f_{g}$ has support in $S$ and there are potentials $\phi_{g}$ and $\mathbf{A}_{g}$ in $H^{2}\left(\mathbb{R}^{3}\right)$ that satisfy Maxwell's equations in the Coulomb gauge.

Proof. Consider a symmetric growing mode of the form $\left(e^{\lambda t} f(x, v), e^{\lambda t} \mathbf{E}(x), e^{\lambda t} \mathbf{B}(x)\right)$ with $\operatorname{Re} \lambda>0$ where $\mathbf{E}, \mathbf{B} \in L_{S}^{2}$ and $f \in L_{x v}^{1} \cap L_{x v}^{\infty}$. We have dropped the subscript $g$. Now $\iint f d v d x=-\int \rho d x$ is an invariant of the linear system and so it must vanish for a growing mode. By the linearized Vlasov equation we have

$$
\lambda f+D f=\mu_{e} \hat{v} \cdot \mathbf{E}-r \mu_{p}\left(\hat{v}_{r} B_{z}-\hat{v}_{z} B_{r}\right) .
$$

Note that the right-hand side $h$ of this equation belongs to $L^{2}\left(\mathbb{R}^{3} \times \mathbb{R}^{3}\right)$ with compact support. Along a particle trajectory we have

$$
\frac{\partial}{\partial s}\left[e^{\lambda s} f(X(s), V(s))\right]=h(X(s), V(s)) .
$$


But $f$ is bounded and $\operatorname{Re} \lambda>0$ so that $e^{\lambda s} f(X, V)$ vanishes as $s \rightarrow-\infty$. Integrating, we therefore have

$$
\begin{aligned}
f & =\mu_{e} \int_{-\infty}^{0} e^{\lambda s} \hat{\mathbf{V}}(s) \cdot \mathbf{E}(X(s)) d s \\
& -\mu_{p} \int_{-\infty}^{0} e^{\lambda s} R(s)\left(\hat{\mathbf{V}}_{r}(s) B_{z}(X(s))-\hat{\mathbf{V}}_{z}(s) B_{r}(X(s))\right) d s .
\end{aligned}
$$

Thus $f(x, v)$ has support in $S$. We define a scalar potential $\phi$ by $\phi(x)=\frac{1}{4 \pi} \int \frac{\rho(y)}{|x-y|} d y$. It follows that $-\Delta \phi=\rho=-\int f d v$ also has compact support. Since $\frac{1}{|x-y|}=\frac{1}{|x|}+$ $O\left(\frac{1}{|x|^{2}}\right)$ for large $x$, we have

$$
\phi(x)=\frac{1}{4 \pi} \frac{1}{|x|} \int \rho(y) d y+O\left(\frac{1}{|x|^{2}}\right)=O\left(\frac{1}{|x|^{2}}\right)
$$

which implies that $\phi \in H^{2}\left(\mathbb{R}^{3}\right)$. Then we define a vector potential $\mathbf{A}$ by solving the equation $\left(\lambda^{2}-\Delta\right) \mathbf{A}=-\lambda \nabla \phi+\mathbf{j} \in L^{2}$. Since $\lambda^{2}$ is not a negative real number, it follows that $\mathbf{A} \in H^{2}\left(\mathbb{R}^{3}\right)$. We have

$$
\left(\lambda^{2}-\Delta\right) \nabla \cdot \mathbf{A}=-\lambda \Delta \phi+\nabla \cdot \mathbf{j}=\lambda \rho+\nabla \cdot \mathbf{j}=0
$$

by the continuity equation, and thus $\nabla \cdot \mathbf{A}=0$. Now we check that $(\phi, \mathbf{A})$ are indeed the potential functions for $\mathbf{E}, \mathbf{B}$. By the Maxwell equations, we have $\lambda E-\nabla \times \mathbf{B}=$ $-\mathbf{j}, \lambda \mathbf{B}+\nabla \times \mathbf{E}=0$ and $\nabla \cdot \mathbf{E}=\rho, \nabla \cdot \mathbf{B}=0$. So $\nabla \cdot(\mathbf{E}+\nabla \phi+\lambda \mathbf{A})=\nabla \cdot \mathbf{E}-\rho=0$ and

$$
\nabla \times(\mathbf{E}+\nabla \phi+\lambda \mathbf{A})=-\lambda(\mathbf{B}-\nabla \times \mathbf{A}) .
$$

By the definition of $\mathbf{A}$

$$
\nabla \times(\mathbf{B}-\nabla \times \mathbf{A})=\lambda \mathbf{E}+\mathbf{j}+\Delta \mathbf{A}=\lambda(\mathbf{E}+\nabla \phi+\lambda \mathbf{A}),
$$

so taking curl of (3.17) we have

$$
(-\Delta+\lambda)(\mathbf{E}+\nabla \phi+\lambda \mathbf{A})=0
$$

which implies that $\mathbf{E}=-\nabla \phi-\lambda \mathbf{A}$. Then by (3.18), $\nabla \times(\mathbf{B}-\nabla \times \mathbf{A})=0$ from which it follows that $\mathbf{B}=\nabla \times \mathbf{A}$ since $\nabla \cdot(\mathbf{B}-\nabla \times \mathbf{A})=\nabla \cdot \mathbf{B}=0$. This ends the proof of the lemma.

To prove Theorem 1.1 (i) on spectral stability, we need to introduce the invariants of the linearized 3D RVM system (3.1), (3.2) and (3.3), for which we introduced the electromagnetic potentials $(\phi, \mathbf{A})$ with the Coulomb gauge $\nabla \cdot \mathbf{A}=0$. The invariants are

$$
\begin{aligned}
I= & \iint_{S}-\frac{1}{\mu_{e}}\left|f+r A_{\theta} \mu_{p}\right|^{2} d x d v+\int\left(|\mathbf{E}|^{2}+|\mathbf{B}|^{2}\right) d x \\
& -\iint r \hat{v}_{\theta} \mu_{p}\left|A_{\theta}\right|^{2} d x d v,
\end{aligned}
$$


where $S$ is the support of $\mu$, and

$$
K_{g}=\iint\left(f+\mu_{e} \hat{v} \cdot \mathbf{A}+r \mu_{p} A_{\theta}\right) g d x d v
$$

for all $g \in \operatorname{ker} D$. The proofs that $I$ and $K_{g}$ are invariant follows by direct calculations which are somewhat similar to the proofs of Lemma 2.7 in $1 \frac{1}{2} \mathrm{D}$ and of Lemma 3.4 below, so we skip them here. Here we assume that $f$ vanishes on the boundary of $S$. This is true for the growing modes, which are our only concern in this paper. In order to prove the stability part of Theorem 1.1, we use the same strategy as in the $1 \frac{1}{2} \mathrm{D}$ case. We begin with a simple lemma.

Lemma 3.3. The projection operator $\mathscr{P}$ maps a function that is odd or even in $\left(v_{r}, v_{z}\right)$ to another function with the same symmetry property.

Proof. First we show that if a function $\xi\left(r, z, v_{r}, v_{\theta}, v_{z}\right)$ is in $\operatorname{ker} D$, then so is $\xi_{-}=$ $\xi\left(r, z,-v_{r}, v_{\theta},-v_{z}\right)$. Set $\tilde{\mathbf{B}}=\mathbf{B}^{0}+\mathbf{B}^{\text {ext }}$ and $\tilde{\mathbf{E}}=\mathbf{E}^{0}+\mathbf{E}^{\text {ext }}$. Then

$$
\begin{aligned}
D= & \hat{v}_{r} \partial_{r}+\hat{v}_{z} \partial_{z}-\left(\tilde{E}_{r}+\hat{v}_{\theta} \tilde{B}_{z}\right) \partial_{v_{r}} \\
& -\left(\hat{v}_{z} \tilde{B}_{r}-\hat{v}_{r} \tilde{B}_{z}\right) \partial_{v_{\theta}}-\left(\tilde{E}_{z}+\hat{v}_{\theta} \tilde{B}_{r}\right) \partial_{v_{z}} .
\end{aligned}
$$

So we have $D\left(\xi_{-}\right)=-\left(D \xi_{-}\right.$and the conclusion follows.

Given a cylindrically symmetric function $h$ odd in $v_{r}$ and $v_{z}$, we want to show that $\mathscr{P} h$ is also odd in $v_{r}$ and $v_{z}$. By definition, for any $\xi \in \operatorname{ker} D$

$$
\iint(h-\mathscr{P} h) \xi d x d v=0 .
$$

Without loss of generality, we may assume $\xi$ to be odd (or even) in $\left(v_{r}, v_{z}\right)$ by the property we have just proved. Then by the change of variable $\left(v_{r}, v_{\theta}\right)$ to $\left(-v_{r},-v_{\theta}\right)$ in (3.19), we have $\iint\left(-h-(\mathscr{P} h)_{-}\right) \xi d v d x=0$. So $(\mathscr{P} h)_{-}=-\mathscr{P} h$. The even case is proven in the same way.

Proof of Theorem 1.1 (i) (Spectral Stability). We merely sketch the proof since it is similar to the $1 \frac{1}{2} \mathrm{D}$ case. For a growing mode $e^{\lambda t}[f(x, v), \mathbf{E}, \mathbf{B}]$ with $\operatorname{Re} \lambda>$ 0 , assume the associated electromagnetic potentials to be $\phi(r, z)$ and $\mathbf{A}(r, z)$. We clearly have the invariant functionals $I=0$ and $K_{g}=0$ for any $g \in \operatorname{ker} D$. Here $I$ takes the form

$$
\begin{aligned}
I(f, \phi, A)= & \iint-\frac{1}{\mu_{e}}\left|f+r A_{\theta} \mu_{p}\right|^{2} d x d v+\int\left(|\nabla \phi|^{2}+\left|\partial_{z} A_{\theta}\right|^{2}+\left|\frac{1}{r} \partial_{r}\left(r A_{\theta}\right)\right|^{2}\right) d x \\
& +\int\left(|\lambda|^{2}|\mathbf{A}|^{2}+\left|\partial_{z} A_{r}-\partial_{r} A_{z}\right|^{2}\right) d x-\iint r \hat{v}_{\theta} \mu_{p}\left|A_{\theta}\right|^{2} d x d v .
\end{aligned}
$$

because $\nabla \cdot \mathbf{A}=0$. Since

$$
\iint\left(f+\mu_{e} \hat{v} \cdot \mathbf{A}+r \mu_{p} A_{\theta}\right) g d x d v=0
$$


for any $g \in \operatorname{ker} D$, there exists a function $h(x, v)$ such that $f+\mu_{e} \hat{v} \cdot \mathbf{A}+r \mu_{p} A_{\theta}=D h$. Then $\int D h d v=\int f d v$ since

$$
\int\left(\mu_{e} \hat{v} \cdot \mathbf{A}+r \mu_{p} A_{\theta}\right) d v=\int\left(\mu_{e} \hat{v}_{\theta} A_{\theta}+r \mu_{p} A_{\theta}\right) d v=A_{\theta} \int \frac{\partial \mu}{\partial v_{\theta}} d v=0,
$$

due to the fact that $\mu_{e}$ is even in $v_{r}$ and $v_{z}$.

Now define the set

$$
F=\left\{\left.h(x, v)\left|\iint \frac{1}{\left|\mu_{e}\right|}\right| D h\right|^{2} d x d v<\infty\right\}
$$

and the functional

$$
J_{A}(h)=\iint \frac{1}{\left|\mu_{e}\right|}\left|D h-\mu_{e} \hat{v} \cdot \mathbf{A}\right|^{2} d x d v+\int|\nabla \phi|^{2} d x
$$

on $F$, where $\Delta \phi=\int f d v=\int D h d v$. Then

$$
\begin{aligned}
I(f, \phi, A) & =J_{A}(h)+\int\left(|\lambda|^{2}|\mathbf{A}|^{2}+\left|\partial_{z} A_{r}-\partial_{r} A_{z}\right|^{2}\right) d x-\iint r \hat{v}_{\theta} \mu_{p}\left|A_{\theta}\right|^{2} d x d v \\
& +\int\left(\left|\partial_{z} A_{\theta}\right|^{2}+\left|\frac{1}{r} \partial_{r}\left(r A_{\theta}\right)\right|^{2}\right) d x .
\end{aligned}
$$

For fixed $\mathbf{A}$, we shall find the minimum of $J_{A}(h)$ on $F$. As in the proof of the $1 \frac{1}{2} \mathrm{D}$ case in Lemma 2.8, the minimizer $h_{0}$ satisfies

$$
\begin{aligned}
D h_{0}= & \mu_{e}(1-\mathscr{P})\left(\hat{v} \cdot \mathbf{A}-\phi^{(0)}\right) \\
= & -\mu_{e} \phi^{(0)}+\mu_{e} \mathscr{P}\left(\phi^{(0)}\right)+\mu_{e} \hat{v}_{\theta} A_{\theta}-\mu_{e} \mathscr{P}\left(\hat{v}_{\theta} A_{\theta}\right) \\
& +\mu_{e}\left(\hat{v}_{r} A_{r}+\hat{v}_{z} A_{z}\right)-\mu_{e} \mathscr{P}\left(\hat{v}_{r} A_{r}+\hat{v}_{z} A_{z}\right)
\end{aligned}
$$

with $\Delta \phi^{(0)}=\int D h_{0} d v$. The function $\hat{v}_{r} A_{r}+\hat{v}_{z} A_{z}$ is odd in $\left(v_{r}, v_{z}\right)$, so it follows that $\mathscr{P}\left(\hat{v}_{r} A_{r}+\hat{v}_{z} A_{z}\right)$ is also odd in $\left(v_{r}, v_{z}\right)$ by Lemma 3.3. Similarly, $\mathscr{P}\left(\hat{v}_{\theta} A_{\theta}\right)$ is even in $\left(v_{r}, v_{z}\right)$. Thus

$$
\int D h_{0} d v=\int\left(-\mu_{e} \phi^{(0)}+\mu_{e} \mathscr{P}\left(\phi^{(0)}\right)+\mu_{e} \hat{v}_{\theta} A_{\theta}-\mu_{e} \mathscr{P}\left(\hat{v}_{\theta} A_{\theta}\right)\right) d v .
$$

Plugging (3.20) into the expression for $J_{A}(h)$, and using similar calculations as in the $1 \frac{1}{2} \mathrm{D}$ case, we have

$$
\begin{aligned}
\min J_{A}(h)= & J_{A}\left(h_{0}\right) \\
= & \iint\left|\mu_{e}\right|(\mathscr{P}(\hat{v} \cdot \mathbf{A}))^{2} d x d v+\left(\left(\mathscr{B}^{0}\right)^{*}\left(\mathscr{A}_{1}^{0}\right)^{-1} \mathscr{B}^{0} A_{\theta}, A_{\theta}\right) \\
= & \iint\left|\mu_{e}\right|\left(\mathscr{P}\left(\hat{v}_{\theta} A_{\theta}\right)\right)^{2} d x d v+\left(\left(\mathscr{B}^{0}\right)^{*}\left(\mathscr{A}_{1}^{0}\right)^{-1} \mathscr{B}^{0} A_{\theta}, A_{\theta}\right) \\
& +\iint\left|\mu_{e}\right|\left(\mathscr{P}\left(\hat{v}_{r} A_{r}+\hat{v}_{z} A_{z}\right)\right)^{2} d x d v .
\end{aligned}
$$


So for a growing mode,

$$
\begin{aligned}
0= & I_{1} \\
\geq & \iint\left|\mu_{e}\right|\left(\mathscr{P}\left(\hat{v}_{r} A_{r}+\hat{v}_{z} A_{z}\right)\right)^{2} d x d v+\left(\mathscr{L}^{0} A_{\theta}, A_{\theta}\right) \\
& \quad+\int\left(|\lambda|^{2}|\mathbf{A}|^{2}+\left|\partial_{z} A_{r}-\partial_{r} A_{z}\right|^{2}\right) d x
\end{aligned}
$$

by the definitions of $\mathscr{L}^{0}$ and $\mathscr{A}_{2}^{0}$. This is impossible if $\mathscr{L}^{0} \geq 0$. Thus $\mathscr{L}^{0} \geq 0$ implies that growing modes cannot exist.

Now we use the splitting technique to study the 3D stability problem. As in the $1 \frac{1}{2} \mathrm{D}$ case, we will prove that the growing mode must be purely growing, and we will control the maximal growth rate. We decompose $f$ as $f=f_{o d}+f_{e v}$ where $f_{\text {od }}\left(f_{\text {ev }}\right)$ is odd (even) in $\left(v_{r}, v_{z}\right)$. Then (3.1) can be written as

$$
\partial_{t} f_{o d}+D f_{e v}=\mu_{e}\left(\hat{v}_{r} E_{r}+\hat{v}_{z} E_{z}\right)-\mu_{p} r\left(\hat{v}_{r} B_{z}-\hat{v}_{z} B_{r}\right)
$$

and

$$
\partial_{t} f_{e v}+D f_{o d}=\mu_{e} \hat{v}_{\theta} E_{\theta}+\mu_{p} r E_{\theta}
$$

So

$$
\begin{aligned}
& \partial_{t}^{2} f_{o d}-D^{2} f_{o d} \\
= & \mu_{e}\left(\hat{v}_{r} E_{t}^{r}+\hat{v}_{z} \partial_{t} E_{z}\right)-\mu_{p} r\left(\hat{v}_{r} \partial_{t} B_{z}-\hat{v}_{z} \partial_{t} B_{r}\right)-\mu_{e} D\left(\hat{v}_{\theta} E^{\theta}\right)-\mu_{p} D\left(r E_{\theta}\right) \\
= & \mu_{e}\left(\hat{v}_{r} \partial_{t} E_{r}+\hat{v}_{z} \partial_{t} E_{z}\right)-\mu_{e} D\left(\hat{v}_{\theta} E_{\theta}\right),
\end{aligned}
$$

or

$$
\partial_{t}^{2} \frac{f_{o d}}{\mu_{e}}=D^{2} \frac{f_{o d}}{\mu_{e}}+\hat{v}_{r} \partial_{t} E_{r}+\hat{v}_{z} \partial_{t} E_{z}-D\left(\hat{v}_{\theta} E_{\theta}\right)
$$

since

$$
\begin{aligned}
D\left(r E_{\theta}\right) & =\hat{v}_{r} \partial_{r}\left(r E^{\theta}\right)+\hat{v}_{z} \partial_{z}\left(r E_{\theta}\right)=-\frac{\partial}{\partial t}\left(\hat{v}_{r} \partial_{r}\left(r A_{\theta}\right)+\hat{v}_{z} \partial_{z}\left(r A_{\theta}\right)\right) \\
& =-r\left(\hat{v}_{r} \partial_{t} B_{z}-\hat{v}_{z} \partial_{t} B_{r}\right)
\end{aligned}
$$

Define

$$
\begin{gathered}
N=\frac{1}{2}\left(\int-\frac{\left|f_{o d}\right|^{2}}{\mu_{e}} d x d v+\int\left|E_{\theta}\right|^{2}+\left|B_{\theta}\right|^{2} d x\right), \\
K=\left(\int-\frac{\left|\partial_{t} f_{o d}\right|^{2}}{\mu_{e}} d x d v+\int\left|\partial_{t} E_{\theta}\right|^{2}+\left|\partial_{t} B_{\theta}\right|^{2} d x\right)
\end{gathered}
$$


and

$$
\begin{aligned}
W & =\iint \frac{1}{\left|\mu_{e}\right|}\left|D f_{o d}-\mu_{e} \hat{v}_{\theta} E_{\theta}\right|^{2} d x d v-\int r \hat{v}_{\theta} \mu_{p}\left|E_{\theta}\right|^{2} d x d v \\
& +\int\left(\left|\frac{1}{r} \partial_{r}\left(r E_{\theta}\right)\right|^{2}+\left|\partial_{z} E_{\theta}\right|^{2}\right) d x+\int\left(\left|\partial_{t} E_{r}\right|^{2}+\left|\partial_{t} E_{z}\right|^{2}\right) d x .
\end{aligned}
$$

Now we state the analogues of Lemmas 2.9 and 2.10.

Lemma 3.4. We have

$$
\frac{d^{2} N}{d t^{2}}=K-W
$$

and

$$
\frac{d}{d t}(K+W)=0
$$

for any complex-valued solution such that $(K+W)(0)<\infty$. Some regularity is required but it is true in particular for any growing mode.

Proof. First we prove (3.22). Multiplying (3.21) by $f_{\text {od }}^{*}$ and integrating, we have

$$
\begin{aligned}
& \iint \frac{\partial_{t}^{2} f_{o d} f_{o d}^{*}}{\mu_{e}} d x d v \\
& =-\iint \frac{\left|D f_{o d}\right|^{2}}{\mu_{e}} d x d v+\iint\left(\hat{v}_{r} \partial_{t} E_{r}+\hat{v}_{z} \partial_{t} E_{z}\right) f_{o d}^{*} d x d v-\iint D\left(\hat{v}_{\theta} E_{\theta}\right) f_{o d}^{*} d x d v .
\end{aligned}
$$

Computing the second integral on the right in (3.24), and using the equation $\partial_{t} \mathbf{E}=$ $-\nabla \partial_{t} \phi-\partial_{t}^{2} \mathbf{A}$, we have

$$
\begin{aligned}
& \iint\left(\hat{v}_{r} \partial_{t} E_{r}+\hat{v}_{z} \partial_{t} E_{z}\right) f_{o d}^{*} d x d v=-\int\left(\partial_{t} E_{r} j_{r}^{*}+\partial_{t} E_{z} j_{z}^{*}\right) d x \\
& =\int\left(\left|\partial_{t} E_{r}\right|^{2}+\left|\partial_{t} E_{z}\right|^{2}\right) d x-\int\left(\partial_{t} E_{r}\left(\nabla \times \mathbf{B}^{*}\right)_{r}+\partial_{t} E_{z}\left(\nabla \times \mathbf{B}^{*}\right)_{z}\right) d x \\
& =\int\left(\left|\partial_{t} E_{r}\right|^{2}+\left|\partial_{t} E_{z}\right|^{2}\right) d x-\left(-\nabla \phi_{t}, \nabla \times \mathbf{B}^{*}\right) \\
& \quad-\int\left(\partial_{t}^{2} A_{r}\left(\partial_{z} B_{\theta}\right)^{*}-\partial_{t}^{2} A_{z} \frac{1}{r} \partial_{r}\left(r B_{\theta}\right)^{*}\right) d x \\
& =\int\left(\left|\partial_{t} E_{r}\right|^{2}+\left|\partial_{t} E^{z}\right|^{2}\right) d x-2 \pi \int\left(\partial_{t}^{2} A_{r} \partial_{z}\left(r B_{\theta}\right)^{*}-\partial_{t}^{2} A_{z} \partial_{r}\left(r B_{\theta}\right)^{*}\right) d r d z \\
& =\int\left(\left|\partial_{t} E_{r}\right|^{2}+\left|\partial_{t} E_{z}\right|^{2}\right) d x+2 \pi \int r \partial_{t}^{2}\left(\partial_{z} A_{r}-\partial_{r} A_{z}\right)\left(B_{\theta}\right)^{*} d r d z \\
& =\int\left(\left|\partial_{t} E_{r}\right|^{2}+\left|\partial_{t} E_{z}\right|^{2}\right) d x+\int \partial_{t}^{2} B_{\theta}\left(B_{\theta}\right)^{*} d x .
\end{aligned}
$$


For the last integral in (3.24) we have

$$
\begin{aligned}
& \iint D\left(\hat{v}_{\theta} E_{\theta}\right) f_{o d}^{*} d x d v=-2 \iint \hat{v}_{\theta} E_{\theta} D f_{o d}^{*} d x d v+\iint \hat{v}_{\theta} E_{\theta} D f_{o d}^{*} d x d v \\
& =-2 \iint \hat{v}_{\theta} E_{\theta} D f_{o d}^{*} d x d v-\iint \hat{v}_{\theta} E_{\theta}\left(\partial_{t} f_{e v}-\mu_{e} \hat{v}_{\theta} E_{\theta}-\mu_{p} r E_{\theta}\right)^{*} d x d v \\
& =-2 \iint \hat{v}_{\theta} E_{\theta} D f_{o d}^{*} d x d v+\iint\left(\hat{v}_{\theta}^{2} \mu_{e}+\hat{v}_{\theta} \mu_{p} r\right)\left|E_{\theta}\right|^{2} d x d v+\int E_{\theta} \partial_{t} j_{\theta}^{*} d x \\
& =-2 \iint \hat{v}_{\theta} E_{\theta} D f_{o d}^{*} d x d v+\iint\left(\hat{v}_{\theta}^{2} \mu_{e}+\hat{v}_{\theta} \mu_{p} r\right)\left|E_{\theta}\right|^{2} d x d v-\int E_{\theta}\left(\partial_{t}^{2} E_{\theta}\right)^{*} d x \\
& -\int\left(\left|\frac{1}{r} \partial_{r}\left(r E_{\theta}\right)\right|^{2}+\left|\partial_{z} E_{\theta}\right|^{2}\right) d x
\end{aligned}
$$

since

$$
\begin{aligned}
\int E_{\theta} \partial_{t} j_{\theta}^{*} d x & =-\int E_{\theta}\left(\partial_{t}^{2} E_{\theta}-\left(\nabla \times \partial_{t} \mathbf{B}\right)_{\theta}\right)^{*} d x \\
& =-\int E_{\theta}\left(\partial_{t}^{2} E_{\theta}\right)^{*} d x-\int E_{\theta}\left((\nabla \times(\nabla \times \mathbf{E}))_{\theta}\right)^{*} d x \\
& =-\int E_{\theta}\left(\partial_{t}^{2} E_{\theta}\right)^{*} d x+\int E_{\theta}\left(\partial_{r}\left(\frac{1}{r} \partial_{r}\left(r E_{\theta}\right)\right)+\partial_{z z} E_{\theta}\right)^{*} d x \\
& =-\int E_{\theta}\left(\partial_{t}^{2} E_{\theta}\right)^{*} d x-\int\left(\left|\frac{1}{r} \partial_{r}\left(r E_{\theta}\right)\right|^{2}+\left|\partial_{z} E_{\theta}\right|^{2}\right) d x
\end{aligned}
$$

So finally from the identity

$$
d^{2} N / d t^{2}=K+\operatorname{Re}\left\{-\iint \frac{\left(\partial_{t}^{2} f_{o d}\right) f_{o d}^{*}}{\mu_{e}} d x d v+\int\left(E_{\theta}\left(\partial_{t}^{2} E_{\theta}\right)^{*}+\left(B_{\theta}\right)^{*} \partial_{t}^{2} B_{\theta}\right) d x\right\}
$$

and the calculations above, we have

$$
\begin{aligned}
d^{2} N / d t^{2}=K+\iint & \frac{\left|D f_{o d}\right|^{2}}{\mu_{e}} d x d v-\int\left(\left|\partial_{t} E_{r}\right|^{2}+\left|\partial_{t} E_{z}\right|^{2}\right) d x \\
& -\int\left(\left|\frac{1}{r} \partial_{r}\left(r E_{\theta}\right)\right|^{2}+\left|\partial_{z} E_{\theta}\right|^{2}\right) d x \\
& \quad-2 \operatorname{Re} \iint \hat{v}_{\theta} E_{\theta} D f_{o d}^{*} d x d v+\iint\left(\hat{v}_{\theta}^{2} \mu_{e}+\hat{v}_{\theta} \mu_{p} r\right)\left|E_{\theta}\right|^{2} d x d v \\
=K+\iint \frac{1}{\mu_{e}}\left|D f_{o d}-\mu_{e} \hat{v}_{\theta} E_{\theta}\right|^{2} d x d v-\int\left(\left|\frac{1}{r} \partial_{r}\left(r E_{\theta}\right)\right|^{2}+\left|\partial_{z} E_{\theta}\right|^{2}\right) d x & \quad-\int\left(\left|\partial_{t} E_{r}\right|^{2}+\left|\partial_{t} E_{z}\right|^{2}\right) d x+\int \hat{v}_{\theta} \mu_{p} r\left|E_{\theta}\right|^{2} d x d v \\
=K-W &
\end{aligned}
$$


Now we prove (3.23). Multiplying (3.21) by $\partial_{t} f_{o d}^{*}$ and integrating, we have

$$
\begin{gathered}
\operatorname{Re} \iint \frac{\partial_{t}^{2} f_{o d} \partial_{t} f_{o d}^{*}}{\mu_{e}} d x d v=\frac{d}{d t} \frac{1}{2} \iint \frac{\left|\partial_{t} f_{o d}\right|^{2}}{\mu_{e}} d x d v \\
=-\frac{d}{d t} \frac{1}{2} \iint \frac{\left|D f_{o d}\right|^{2}}{\mu_{e}} d x d v+\operatorname{Re} \iint\left(\hat{v}_{r} \partial_{t} E_{r}+\hat{v}_{z} \partial_{t} E_{z}\right) \partial_{t} f_{o d}^{*} d x d v \\
-\operatorname{Re} \iint D\left(\hat{v}_{\theta} E_{\theta}\right) \partial_{t} f_{o d}^{*} d x d v .
\end{gathered}
$$

By the same calculations as above, the second term is

$$
\operatorname{Re} \iint\left(\hat{v}_{r} \partial_{t} E_{r}+\hat{v}_{z} \partial_{t} E_{z}\right) \partial_{t} f_{o d}^{*} d x d v=\frac{d}{d t} \frac{1}{2} \int\left(\left|\partial_{t} E_{r}\right|^{2}+\left|\partial_{t} E_{z}\right|^{2}+\left|\partial_{t} B_{\theta}\right|^{2}\right) d x
$$

and the third term is

$\operatorname{Re} \iint D\left(\hat{v}_{\theta} E_{\theta}\right) \partial_{t} f_{o d}^{*} d x d v=-\frac{d}{d t} \operatorname{Re} \iint \hat{v}_{\theta} E_{\theta} D f_{o d}^{*} d x d v+\operatorname{Re} \iint \hat{v}_{\theta} \partial_{t} E_{\theta} D f_{o d}^{*} d x d v$

The last term is

$$
\begin{aligned}
& \operatorname{Re} \iint \hat{v}_{\theta} \partial_{t} E_{\theta} D f_{o d}^{*} d x d v \\
= & -\operatorname{Re} \iint \hat{v}_{\theta} \partial_{t} E_{\theta}\left(\partial_{t} f_{e v}-\mu_{e} \hat{v}_{\theta} E_{\theta}-\mu_{p} r E_{\theta}\right)^{*} d x d v \\
= & \frac{d}{d t} \frac{1}{2} \iint\left(\hat{v}_{\theta}^{2} \mu_{e}+\hat{v}_{\theta} \mu_{p} r\right)\left|E_{\theta}\right|^{2} d x d v+\operatorname{Re} \int \partial_{t} E_{\theta} \partial_{t} j_{\theta}^{*} d x \\
= & \frac{1}{2} \frac{d}{d t} \iint\left(\hat{v}_{\theta}^{2} \mu_{e}+\hat{v}_{\theta} \mu_{p} r\right)\left|E_{\theta}\right|^{2} d x d v \\
& \quad-\frac{1}{2} \frac{d}{d t} \int\left(\left|\partial_{t} E_{\theta}\right|^{2}+\left|\frac{1}{r} \partial_{r}\left(r E_{\theta}\right)\right|^{2}+\left|\partial_{z} E_{\theta}\right|^{2}\right) d x
\end{aligned}
$$


Combining these calculations, we deduce the invariance of the expression

$$
\begin{aligned}
& \frac{1}{2} \iint \frac{\left|\partial_{t} f_{o d}\right|^{2}}{\mu_{e}} d x d v+\frac{1}{2} \iint \frac{\left|D f_{o d}\right|^{2}}{\mu_{e}} d x d v-\frac{1}{2} \int\left(\left|\partial_{t} E_{r}\right|^{2}+\left|\partial_{t} E_{z}\right|^{2}+\left|\partial_{t} B_{\theta}\right|^{2}\right) d x \\
& -\operatorname{Re} \iint \hat{v}_{\theta} E_{\theta} D f_{o d}^{*} d x d v+\frac{1}{2} \iint\left(\hat{v}_{\theta}^{2} \mu_{e}+\hat{v}_{\theta} \mu_{p} r\right)\left|E_{\theta}\right|^{2} d x d v \\
& -\frac{1}{2} \int\left(\left|\partial_{t} E_{\theta}\right|^{2}+\left|\frac{1}{r} \partial_{r}\left(r E_{\theta}\right)\right|^{2}+\left|\partial_{z} E_{\theta}\right|^{2}\right) d x \\
& =\frac{1}{2} \iint \frac{\left|\partial_{t} f_{o d}\right|^{2}}{\mu_{e}} d x d v-\frac{1}{2} \int\left(\left|\partial_{t} B_{\theta}\right|^{2}+\left|\partial_{t} E_{\theta}\right|^{2}\right) d x \\
& +\frac{1}{2} \iint \frac{\left|D f_{o d}-\mu_{e} \hat{v}_{\theta} E_{\theta}\right|^{2}}{\mu_{e}} d x d v-\frac{1}{2} \int\left(\left|\partial_{t} E_{r}\right|^{2}+\left|\partial_{t} E_{z}\right|^{2}\right) d x \\
& +\iint r \hat{v}_{\theta} \mu_{p}\left|E_{\theta}\right|^{2} d x d v-\frac{1}{2} \int\left(\left|\frac{1}{r} \partial_{r}\left(r E_{\theta}\right)\right|^{2}+\left|\partial_{z} E_{\theta}\right|^{2}\right) d x \\
& =-\frac{1}{2} K-\frac{1}{2} W \text {. }
\end{aligned}
$$

Thus (3.23) is proven.

Proof of Theorem 1.1 (completed). Theorem 1(i) has already been proven. As in the $1 \frac{1}{2} \mathrm{D}$ case, we check that for a $3 \mathrm{D}$ growing mode $(K+W)(0)<\infty$, so that Lemma 3.4 is applicable. The purely growing property, Theorem 1.1(ii), is proven just as in Theorem 2.1(ii). Now

$$
\Delta \partial_{t} \phi=\int \partial_{t} f d v=-\int D f_{o d} d v
$$

Following the same kinds of calculations as in the proof of spectral stability for Theorem 1.1(i), we deduce that $W \geq\left(\mathscr{L}^{0} E_{\theta}, E_{\theta}\right)$. The estimate of the maximal growth rate, Theorem 1.1(iii), follows exactly as in the $1 \frac{1}{2} \mathrm{D}$ case.

\section{Examples in the $1 \frac{1}{2} \mathbf{D}$ case}

In this section, we apply our stability criterion to various examples in $1 \frac{1}{2} \mathrm{D}$ for the two species case. We use the notation of the introduction. Recall that Theorems 1.2 and 1.3 tell us that if the operator $\mathscr{L}^{0}$ (defined by (1.16)) is nonnegative, then the equilibrium $\left(f^{0, \pm}, \psi^{0}, \phi^{0}\right)$ is spectrally stable. If $\mathscr{L}^{0}$ has an even eigenfunction for a negative eigenvalue, then there exists a growing mode. In the following, we use this stability criterion for two simple cases: homogeneous equilibria and purely magnetic equilibria. We will begin with a remark about the sharp stability criterion for a general homogeneous equilibria, improving the results in [15]. 
For purely magnetic equilibria, the operator $\mathscr{L}^{0}$ has the simple form of a SturmLiouville operator plus a positive nonlocal term. Using this simpler stability criterion, we can easily prove linear instability of weakly inhomogeneous purely magnetic equilibrium under perturbations of double the period by perturbing from the unstable homogeneous equilibrium. However, the construction of a stable inhomogeneous equilibrium is quite involved. We will construct an explicit example for which the stabilizing nonlocal term can be estimated and the stability condition can be verified.

\subsection{Stability criterion for homogeneous equilibria}

As the first, rather simple application of our stability criterion $\mathscr{L}^{0} \geq 0$, we consider a homogeneous plasma with $f^{0, \pm}=\mu^{ \pm}\left(\langle v\rangle, v_{2}\right)$ and $\mathbf{E}^{0}=\mathbf{B}^{0}=0$. Assume $0<\mu^{ \pm} \in C^{2}$ and $\mu_{e}^{ \pm}<0$. If one assumes additionally that $\mu^{ \pm}$is even in $v_{2}$ and that

$$
D_{0}=\left(\frac{2 \pi}{P_{0}}\right)^{2}=\int_{\mathbf{R}^{2}} \sum_{ \pm} \hat{v}_{2} \mu_{p}^{ \pm}\left(\langle v\rangle, v_{2}\right) d v>0
$$

then in [15] it was shown that the steady state is unstable (both linearly and nonlinearly) if the perturbation period $P>P_{0}$. It was also claimed in [15] that if $P>P_{0}$, then the steady state is nonlinearly stable. However in that proof the evenness of the magnetic field perturbation $B$ was implicitly assumed so that the stability obtained was only conditional. Below, we will deduce the sharp stability criterion for a general equilibrium without an evenness assumption. We shall see that, even to get linear stability for a homogeneous equilibrium, the nonlocal term involving $\mathscr{P}^{ \pm}$in $\mathscr{L}^{0}$ is indispensable.

In the homogeneous case, $D^{ \pm}=\hat{v}_{1} \partial_{x}$ so that $\mathscr{P}^{ \pm}[\eta(v) h(x)]=\eta(v) \mathscr{Q} h$ where $\mathscr{Q} h=\bar{h}$ denotes the average of $h$ over the perturbation period $P$. Denote

$$
\begin{aligned}
& D_{1}=\sum_{ \pm} \int \mu_{p}^{ \pm} d v, \quad D_{2}=\sum_{ \pm} \int \hat{v}_{2} \mu_{e}^{ \pm} d v=-D_{1}, \\
& D_{3}=-\sum_{ \pm} \int \mu_{e}^{ \pm} d v>0, \quad D_{4}=-\sum_{ \pm} \int \mu_{e}^{ \pm}\left(\hat{v}_{2}\right)^{2} d v>0,
\end{aligned}
$$

where $\mu^{ \pm}=\mu^{ \pm}\left(\langle v\rangle, v_{2}\right)$. Then we have

$$
\mathscr{L}^{0}=\left(D_{1}+D_{2} \mathscr{Q}\right)\left(-\partial_{x}^{2}+D_{3}-D_{3} \mathscr{Q}\right)^{-1}\left(D_{1}+D_{2} \mathscr{Q}\right)-\partial_{x}^{2}-D_{0}+D_{4} \mathscr{Q} .
$$


Clearly we can study the properties of $\mathscr{L}^{0}$ for each Fourier mode $e^{i k 2 \pi x / P}$ separately. For $k=0$, we have

$$
\begin{aligned}
\mathscr{L}^{0}(1) & =D_{4}-D_{0}=-\int_{\mathbf{R}^{2}} \sum_{ \pm} \hat{v}_{2}\left(\mu_{p}^{ \pm}+\mu_{e}^{ \pm} \hat{v}_{2}\right) d v \\
& =-\int_{\mathbf{R}^{2}} \sum_{ \pm} \hat{v}_{2} \frac{\partial \mu^{ \pm}}{\partial v_{2}}=\int_{\mathbf{R}^{2}} \sum_{ \pm} \mu^{ \pm} \partial_{v_{2}}\left(\hat{v}_{2}\right) d v \\
& =\int_{\mathbf{R}^{2}} \sum_{ \pm} \mu^{ \pm} \partial_{v_{2}}^{2}\left(\sqrt{1+|v|^{2}}\right) d v>0 .
\end{aligned}
$$

For $k \neq 0$, we have $\mathscr{Q}\left(e^{i k \frac{2 \pi}{P} x}\right)=0$ and

$$
\mathscr{L}^{0}\left(e^{i k \frac{2 \pi}{P} x}\right)=\left(D_{1}^{2}\left(\left(k \frac{2 \pi}{P}\right)^{2}+D_{3}\right)^{-1}+\left(k \frac{2 \pi}{P}\right)^{2}-D_{0}\right) e^{i k \frac{2 \pi}{P} x}=\lambda_{k} e^{i k \frac{2 \pi}{P} x} .
$$

The condition for $\lambda_{k} \geq 0$ is that either

$$
\left(D_{3}+D_{0}\right)^{2}-4 D_{1}^{2}<0
$$

or

$$
\left(D_{3}+D_{0}\right)^{2}-4 D_{1}^{2} \geq 0 \text { and } k^{2}\left(\frac{2 \pi}{P}\right)^{2}>\frac{D_{0}-D_{3}+\sqrt{\left(D_{3}+D_{0}\right)^{2}-4 D_{1}^{2}}}{2} .
$$

We can always choose the even eigenfunction $\cos (k 2 \pi x / P)$ for $\mathscr{L}^{0}$ in this case, so that Theorem 2.2 is applicable. Summarizing the above computations, we have the following.

Theorem 4.1. Consider a homogeneous equilibrium with $f^{0, \pm}=\mu^{ \pm}\left(\langle v\rangle, v_{2}\right)$. Assume $\mu_{e}^{ \pm}<0$ and $\int\left(\left|\mu_{e}^{ \pm}\right|+\left|\mu_{p}^{ \pm}\right|\right) d v \leq \infty$. Then the sharp linear stability condition is that either

$$
\left(D_{3}+D_{0}\right)^{2}-4 D_{1}^{2}<0
$$

or

$$
\left(D_{3}+D_{0}\right)^{2}-4 D_{1}^{2} \geq 0 \text { and }\left(\frac{2 \pi}{P}\right)^{2} \geq \frac{D_{0}-D_{3}+\sqrt{\left(D_{3}+D_{0}\right)^{2}-4 D_{1}^{2}}}{2} .
$$

Remark. This theorem shows that if

$$
\left(D_{3}+D_{0}\right)^{2}-4 D_{1}^{2}<0
$$

or

$$
\left(D_{3}+D_{0}\right)^{2}-4 D_{1}^{2} \geq 0 \text { and } 0 \geq \frac{D_{0}-D_{3}+\sqrt{\left(D_{3}+D_{0}\right)^{2}-4 D_{1}^{2}}}{2}
$$


then the equilibrium is linearly stable to arbitrary perturbations of any period $P$. If

$$
\left(D_{3}+D_{0}\right)^{2}-4 D_{1}^{2} \geq 0 \text { and } D_{5}:=\frac{D_{0}-D_{3}+\sqrt{\left(D_{3}+D_{0}\right)^{2}-4 D_{1}^{2}}}{2}>0,
$$

then the critical period is $P_{c r}=\frac{2 \pi}{\sqrt{D_{5}}}$ The equilibrium is linearly stable iff $P \leq P_{c r}$.

We note that in case $\mu^{ \pm}$are even (in $v_{2}$ ), then $D_{1}=0$. So if $D_{0} \leq 0$, the equilibrium is linearly stable to perturbations of any period. If $D_{0}>0$, then $D_{5}=$ $D_{0}$ and $P_{c r}=\frac{2 \pi}{\sqrt{D_{0}}}$. In this case, linear instability follows if $P>\frac{2 \pi}{\sqrt{D_{0}}}$ which recovers the instability result in [15]. We also get the linear stability for $P \leq \frac{2 \pi}{\sqrt{D_{0}}}$, improving the conditional stability result in [15].

\subsection{Stability criterion for purely magnetic equilibria}

Here we consider the so-called purely magnetic case for which the steady electric field vanishes. In this case, the stability criterion takes a much simpler form. For the purely magnetic case we make the assumption that

$$
\mu^{+}(e, p)=\mu^{-}(e,-p)
$$

We claim that there exists a solution with $\phi^{0} \equiv 0$ provided $\psi^{0}$ satisfies the ODE

$$
\partial_{x}^{2} \psi^{0}=2 \int \hat{v}_{2} \mu^{-}\left(\langle v\rangle, v_{2}-\psi^{0}(x)\right) d v .
$$

Indeed, let $R$ be the operator that reverses the momentum, that is, $R f(x, v)=$ $f(x,-v)$. Then with $\phi^{0} \equiv 0$ we have

$$
R\left[\mu^{-}\left(\langle v\rangle, v_{2}-\psi^{0}\right)\right]=\mu^{-}\left(\langle v\rangle,-v_{2}-\psi^{0}\right)=\mu^{+}\left(\langle v\rangle, v_{2}+\psi^{0}\right)
$$

so that

and

$$
\rho^{0}=\int\left(\mu^{+}-\mu^{-}\right) d v=\int\left(R \mu^{-}-\mu^{-}\right) d v=0
$$

$\partial_{x}^{2} \psi^{0}=-j_{2}^{0}=-\int \hat{v}_{2}\left(\mu^{+}-\mu^{-}\right) d v=-\int \hat{v}_{2}\left(R \mu^{-}-\mu^{-}\right) d v=2 \int \hat{v}_{2} \mu^{-}\left(\langle v\rangle, v_{2}-\psi^{0}\right) d v$.

This proves the claim.

Let $T_{\psi^{0}}$ be the minimal period of the periodic solution $\psi^{0}$ to (4.2). By adjusting its starting point, we can always arrange that the solution satisfies the conditions

$$
\begin{gathered}
\psi^{0}(0)=\psi^{0}\left(T_{\psi^{0}}\right)=\min _{0 \leq x \leq T_{\psi^{0}}} \psi^{0}(x), \quad \psi^{0}\left(\frac{T_{\psi^{0}}}{2}\right)=\max _{0 \leq x \leq T_{\psi^{0}}} \psi^{0}(x), \\
\psi^{0}(x)=\psi^{0}\left(T_{\psi^{0}}-x\right), \quad \forall x \in\left[0, T_{\psi^{0}}\right],
\end{gathered}
$$

with $\psi^{0}(x)$ strictly increasing in $\left[0, T_{\psi^{0} / 2}\right]$. Then $\partial_{x} \psi^{0}$ has exactly two zeros per period. (In the Appendix, we also construct truly electromagnetic equilibria with $\mathbf{E}^{0} \neq 0$ such that $\psi^{0}$ has the same structure.) 
For a purely magnetic equilibrium, the trajectory equation reduces to

$$
\dot{X}^{ \pm}=\hat{V}_{1}^{ \pm}, \quad \dot{V}_{1}^{ \pm}=\mp \hat{V}_{2}^{ \pm} \partial_{x} \psi^{0}\left(X^{ \pm}\right), \quad \dot{V}_{2}^{ \pm}= \pm \hat{V}_{1}^{ \pm} \partial_{x} \psi^{0}\left(X^{ \pm}\right)
$$

and it is easy to check that $X^{-}(-s ; x,-v)=X^{+}(s ; x, v)$ and $V^{-}(-s ; x,-v)=-V^{+}(s ; x, v)$.

We also have $R \mathscr{P}^{-}=\mathscr{P}^{+} R$ in the purely magnetic case. [Indeed, $f \in k e r D^{-}$iff $R f \in \operatorname{ker}^{+}$. Hence $\mathscr{P}^{-} f=f$ iff $\mathscr{P}^{+} R f=R f$.] For brevity, we shall write

$$
\mu^{+}=R \mu^{-}, \mu_{e}^{+}=R \mu_{e}^{-}, \mu_{p}^{+}=-R \mu_{p}^{-} .
$$

Lemma 4.2. For a purely magnetic equilibrium satisfying (4.1) and (4.2), we have $\mathscr{B}^{0}=0$ and hence $\mathscr{L}^{0}=\mathscr{A}_{2}^{0}$. Furthermore,

$$
\mathscr{A}_{2}^{0} h=-\partial_{x}^{2} h-2 \int \hat{v}_{2} \mu_{p}^{-} d v h-2 \int \mu_{e}^{-} \hat{v}_{2} \mathscr{P}^{-}\left(\hat{v}_{2} h\right) d v,
$$

where $\mu^{ \pm}=\mu^{ \pm}\left(\langle v\rangle, v_{2}-\psi^{0}(x)\right)$.

Proof. For any $h \in L_{P}^{2}$, we calculate

$$
\begin{aligned}
B^{0} h & =\left(\sum_{ \pm} \int \mu_{p}^{ \pm} d v\right) h+\sum_{ \pm} \int \mu_{e}^{ \pm} \mathscr{P}^{ \pm}\left(\hat{v}_{2} h\right) d v \\
& =\left(\int\left(\mu_{p}^{-}+R \mu_{p}^{+}\right) d v\right) h+\int\left(\mu_{e}^{-} \mathscr{P}^{-}\left(\hat{v}_{2} h\right)+R \mu_{e}^{+} R \mathscr{P}^{+}\left(\hat{v}_{2} h\right)\right) d v
\end{aligned}
$$

by changing variables $v \rightarrow-v$ in two of the + integrals. Using $R \mathscr{P}^{-}=\mathscr{P}^{+} R$ and (4.3), we end up with complete cancelation so that $B^{0}=0$. In exactly the same way, noticing that $R\left(\hat{v}_{2} \mu_{p}^{+}\right)=-\hat{v}_{2} R\left(\mu_{p}^{+}\right)=\hat{v}_{2} \mu_{p}^{-}$and $R\left(\mu_{e}^{+} \hat{v}_{2}\right)=-\hat{v}_{2} R\left(\mu_{e}^{+}\right)=$ $-\hat{v}_{2} \mu_{e}^{-}$, we calculate

$$
\begin{aligned}
\mathscr{A}_{2}^{0} h & =-\partial_{x}^{2} h-\int\left(R\left(\hat{v}_{2} \mu_{p}^{+}\right)+\hat{v}_{2} \mu_{p}^{-}\right) d v h-\int\left(R\left(\mu_{e}^{+} \hat{v}_{2}\right) R \mathscr{P}^{+}\left(\hat{v}_{2} h\right)+\mu_{e}^{-} \hat{v}_{2} \mathscr{P}^{-}\left(\hat{v}_{2} h\right)\right) \\
& =-\partial_{x}^{2} h-2 \int \hat{v}_{2} \mu_{p}^{-} d v h-2 \int \hat{v}_{2} \mu_{e}^{-} \mathscr{P}^{-}\left(\hat{v}_{2} h\right) d v .
\end{aligned}
$$

Lemma 4.3. Consider the operator $\mathscr{L}^{0}$ to be defined on the space of $T_{\psi^{0}}$-periodic functions. If $\mathscr{L}^{0}$ has a negative eigenvalue, then its ground state is an even function of $x$.

Proof. For brevity, we now write $\mu=\mu^{-}$and $\mathscr{P}=\mathscr{P}^{-}$. We also write $\mathscr{L}^{0}=$ $\mathscr{M}+\mathscr{N}$ where

$$
\mathscr{M} h=\left[-\partial_{x}^{2}-2 \int \hat{v}_{2} \mu_{p} d v\right] h, \quad \mathscr{N} h=-2 \int \mu_{e} \hat{v}_{2} \mathscr{P}\left(\hat{v}_{2} h\right) d v .
$$

Differentiating the equation satisfied by $\psi^{0}$, we find that $\mathscr{M}\left(\partial_{x} \psi^{0}\right)=0$. Since $\mathscr{M}$ is a standard second-order operator, its ground state is even and non-vanishing. 
Since $\partial_{x} \psi^{0}$ is odd with exactly two zeros per period, $\mathscr{M}$ must have exactly one negative eigenvalue. Moreover, $\mathscr{N} \geq 0$ since

$$
(\mathscr{N} h, h)=-2 \int \mu_{e}\left|\mathscr{P}\left(\hat{v}_{2} h\right)\right|^{2} \geq 0 .
$$

We claim that if $h(x)$ is an even function, then $\mathscr{P}\left(\hat{v}_{2} h\right)$ is an even function of $x$ for each $v$. To prove this, recall that $D=D^{-}=\hat{v}_{1} \partial_{x}-\partial_{x} \psi^{0}\left(\hat{v}_{1} \partial_{v_{2}}-\hat{v}_{2} \partial_{v_{1}}\right)$. Thus $h$ and $\mathscr{P} h$ have opposite parity. Now $\mu_{e} \hat{v}_{2} h-\mathscr{P}\left(\mu_{e} \hat{v}_{2} h\right) \in(\text { kerD })^{\perp}$. Let $h$ be even, say. Changing $x$ to $-x$, we have $\left(\mu_{e} \hat{v}_{2} h\right)(x, v)-\mathscr{P}\left(\mu_{e} \hat{v}_{2} h\right)(-x, v) \in(k e r D)^{\perp}$. Thus $\mathscr{P}\left(\mu_{e} \hat{v}_{2} h\right)-\mathscr{P}\left(\mu_{e} \hat{v}_{2} h\right)(-x, v) \in(\operatorname{ker} D)^{\perp} \cap \operatorname{ker} D=\{0\}$. So $\mathscr{P}\left(\mu_{e} \hat{v}_{2} h\right)$ is even. Similarly, if $h$ is odd, so is $\mathscr{P}\left(\hat{v}_{2} h\right)$. This proves the claim.

Now let $-\lambda$ be the lowest eigenvalue of $\mathscr{L}^{0}$, assumed negative. Let $h$ be the corresponding eigenfunction of unit $L^{2}$ norm. Since $\mathscr{L}^{0}$ preserves parity, $\left(\mathscr{L}^{0} h, h\right)=\left(\mathscr{L}^{0} h_{e}, h_{e}\right)+\left(\mathscr{L}^{0} h_{o}, h_{o}\right)$, where $h_{e}$ is the even part of $h$ and $h_{o}$ is the odd part of $h$. Thus

$-\lambda\left[1-\left(h_{o}, h_{o}\right)\right]=-\lambda\left(h_{e}, h_{e}\right) \leq\left(\mathscr{L}^{0} h_{e}, h_{e}\right)=\left(\mathscr{L}^{0} h, h\right)-\left(\mathscr{L}^{0} h_{o}, h_{o}\right)=-\lambda-\left(\mathscr{L}^{0} h_{o}, h_{o}\right)$,

whence $\left(\mathscr{L}^{0} h_{o}, h_{o}\right) \leq-\lambda\left(h_{o}, h_{o}\right)$. Therefore either $h_{o}$ is a ground state or else $h_{o}=0$. Similarly, the same is true of $h_{e}$. Now in case $v$ were a normalized ground state that is odd, then $-\lambda=\left(\mathscr{L}^{0} v, v\right)=(\mathscr{M} v, v)+(\mathscr{N} v, v) \geq(\mathscr{M} v, v)$ so that $\mathscr{M}$ would have a negative eigenvalue. However, $\mathscr{M}$ has only one negative eigenvalue and its eigenfunction (the ground state) $w$ is even and non-vanishing. Since $v$ and $w$ are orthogonal, this is a contradiction. Therefore any ground state of $\mathscr{L}^{0}$ must be even.

By this lemma, all we have to check for linear stability is the nonnegativity of the operator $\mathscr{L}^{0}=\mathscr{A}_{2}^{0}$ restricted to the even functions. Moreover, combining this lemma with Theorems 1.2 and 1.3 , we will deduce that $\mathscr{L}^{0} \geq 0$ is the sharp linear stability criterion for a purely magnetic equilibrium of minimum period.

\subsection{An unstable purely magnetic equilibrium}

Now we construct an unstable purely magnetic equilibrium with a small magnetic field. The idea is to perturb the unstable homogeneous equilibrium to get an unstable weakly inhomogeneous equilibrium. A tricky perturbation argument was developed in [11] to get an unstable electrostatic BGK wave of small amplitude, but with our explicit stability criterion $\mathscr{A}_{2}^{0} \geq 0$, the perturbation argument here becomes almost trivial. We begin with a periodic purely magnetic equilibrium satisfying (4.2) where $\mu$ satisfies:

$$
\mu \in C^{1}, \mu_{e}<0 \text { and } D_{0}=2 \int \hat{v}_{2} \mu_{p}\left(\langle v\rangle, v_{2}\right) d v>0 .
$$

Assume $\mu^{+}=\mu^{-}$is even. If we write (4.2) as $\partial_{x}^{2} \psi^{0}=g\left(\psi^{0}\right)$, then $g(0)=0$ by oddness and $g^{\prime}(0)<0$. Hence the origin is a center. So there exists a family of periodic solutions $\psi_{\varepsilon}^{0}\left(0<\varepsilon \leq \varepsilon_{0}\right)$ with periods $T_{\psi_{\varepsilon}^{0}}$ satisfying (4.2) such that 
i) $\left|\psi_{\varepsilon}^{0}\right|_{C^{1}} \rightarrow 0$ as $\varepsilon \rightarrow 0+$ and

ii) $T_{\psi_{\varepsilon}^{0}} \rightarrow P_{c r}=\frac{2 \pi}{\sqrt{D_{0}}}$.

Each $\psi_{\varepsilon}^{0}$ gives us a periodic equilibrium.

Theorem 4.4. For $\varepsilon$ sufficiently small, this periodic equilibrium is linearly unstable under $2 T_{\psi_{\varepsilon}^{0}}$-periodic perturbations.

Proof. We use $\mathscr{A}_{2}^{0, \varepsilon}$ to denote the stability operator for the equilibrium with parameter $\varepsilon$. By Theorem 4.1 or by the instability result in [15], the homogeneous equilibrium with $f^{ \pm}=\mu\left(\langle v\rangle, v_{2}\right)$ and $\mathbf{E}^{0}=B^{0}=0$ is unstable under $2 P_{c r}$-periodic perturbations. In particular, if we choose the $2 P_{c r}$-periodic function $\phi^{-, 0}=\cos \left(\pi x / P_{c r}\right)$, then $\left(\mathscr{A}_{2}^{0,0} \phi^{-, 0}, \phi^{-, 0}\right)<0$. Set $\phi^{-, \varepsilon}=\cos \left(\pi x / T_{\psi_{\varepsilon}^{0}}\right)$ which is even and has period $2 T_{\psi_{\varepsilon}^{0}}$. Using the same argument as in [24] for the 1D Vlasov-Poisson case, we have $\left(\mathscr{A}_{2}^{0, \varepsilon} \phi^{-, \varepsilon}, \phi^{-, \varepsilon}\right)<0$ for $\varepsilon$ small enough. By considering $\mathscr{A}_{2}^{0, \varepsilon}$ on the space of even functions, it follows that $\mathscr{A}_{2}^{0, \varepsilon}$ has an even eigenfunction corresponding to a negative eigenvalue when $\varepsilon$ is small. By Theorem 1.3 and Lemma 4.3, this implies the existence of a growing mode for the $\varepsilon$-equilibrium with magnetic potential $\psi_{\varepsilon}^{0}$.

Remark. The form of $\mathscr{A}_{2}^{0}$ looks very similar to the operator $\mathscr{A}^{0}$ used in [20] to study the linear instability of periodic BGK waves of the 1D Vlasov-Poisson system. In [20], linear instability under a double-periodic perturbation is proven for any periodic BGK wave by constructing a double-periodic test function $\phi^{-}$such that $\left(\mathscr{A}^{0} \phi^{-}, \phi^{-}\right)<0$. However, the construction in [20] cannot be carried over to the current purely magnetic case because of the more complicated trajectory structure. We conjecture that, by using a more delicate construction, an arbitrary periodic purely magnetic wave can still be proven to be unstable under perturbations of double the period.

\subsection{A stable purely magnetic equilibrium}

The explicit construction of a stable inhomogeneous example is much more involved. We consider a periodic purely magnetic equilibrium under perturbations of minimal period $P$. As remarked above, it is quite possible that any periodic purely magnetic equilibrium is unstable under multiply-periodic perturbations, that is, under perturbations that are allowed to have a period that is a multiple of $P$. To see the difficulty of proving stability, we recall that the linear stability condition for a purely magnetic equilibrium is that $\mathscr{L}^{0}=\mathscr{A}_{2}^{0}=\mathscr{M}+\mathscr{N} \geq 0$. But the SturmLiouville operator $\mathscr{M}$ has a negative eigenvalue, since $\mathscr{M} \psi_{x}^{0}=0$ and $\psi_{x}^{0}$ has a zero. So in order to get stability we must use the positive operator $\mathscr{N}$ to balance the negative part of $\mathscr{M}$. Because the operator $\mathscr{N}$ is highly nonlocal, the construction of a stable example is rather tricky. 
We choose $\mu=\mu^{-}=\mu^{+}=C \sigma(e)\left(1+p^{2}\right)$ where $C$ is a constant and either $\sigma>0, \sigma^{\prime}<0$ or $\sigma \geq 0$ with $\sigma^{\prime}<0$ on $\{\sigma>0\}$. Then

$$
\partial_{x}^{2} \psi^{0}=2 \int \hat{v}_{2} C \sigma(\langle v\rangle)\left(1+\left(v_{2}-\psi^{0}(x)\right)^{2}\right) d v=-4 C \int \hat{v}_{2} v_{2} \sigma(\langle v\rangle) d v \psi^{0}(x) .
$$

Because the last integral is positive, we can choose $C$ so that $\partial_{x}^{2} \psi^{0}=-\psi^{0}$. We then choose $\psi^{0}(x)=\varepsilon \cos x$, which of course has period $2 \pi$.

To indicate the dependence on $\varepsilon$, we now write $\mathscr{L}^{0}=\mathscr{L}(\varepsilon)=\mathscr{M}(\varepsilon)+\mathscr{N}(\varepsilon)$, where

$$
\mathscr{M}(\varepsilon)=-\partial_{x}^{2}-2 \int \hat{v}_{2} \mu_{p} d v=-\partial_{x}^{2}-4 C \int \hat{v}_{2} \sigma(\langle v\rangle)\left(v_{2}-\varepsilon \cos x\right) d v=-\partial_{x}^{2}-1
$$

because of the definition of $C$ and the oddness with respect to $v_{2}$ of the term with $\varepsilon$. Denote $\mu_{e}=\mu_{e}^{\varepsilon,-}=\sigma^{\prime}\left(\langle v\rangle\left(1+\left(v_{2}-\varepsilon \cos x\right)^{2}\right)\right.$. Then

$$
\mathscr{N}(\varepsilon) h=-2 \int \hat{v}_{2} \mu_{e} \mathscr{P}_{\varepsilon}^{-}\left[\hat{v}_{2} h\right] d v .
$$

The parameter $\varepsilon$ appears both as the coefficient of the cosine and within the projection $\mathscr{P}_{\varepsilon}^{-}$. Since $C_{1} \sigma(\langle v\rangle)\left(1+v_{2}^{2}\right) \leq\left|\mu_{e}\right| \leq C_{2} \sigma(\langle v\rangle)\left(1+v_{2}^{2}\right)$ for some constants $C_{1}, C_{2}>0$, we can consider $\mathscr{P}_{\varepsilon}^{-}$to be the projection from the $\mu^{0}$-weighted $L_{x, v}^{2}$ space $L_{\mu^{0}}^{2}$ to $\operatorname{ker} D_{\varepsilon}$, where $\mu^{0}=\sigma(\langle v\rangle)\left(1+v_{2}^{2}\right)$ and $D_{\varepsilon}=\hat{v}_{1} \partial_{x}-\varepsilon \hat{v}_{2} B_{0} \partial_{v_{1}}+$ $\varepsilon \hat{v}_{1} B_{0} \partial_{v_{2}}$ with $B_{0}=\partial_{x} \psi^{0}(x)=-\sin x$.

In the limiting case $\varepsilon=0, D_{0}=\hat{v}_{1} \partial_{x}$ so that $\mathscr{P}_{0}^{-}[\eta(v) h(x)]=\eta(v) \bar{h}$ where $\bar{h}$ denotes the average of $h$ over a period of length $2 \pi$. So the limiting operator of $\mathscr{N}$ is

where

$$
\mathscr{N}(0) h=\lim _{\varepsilon \rightarrow 0} \mathscr{N}(\varepsilon)=-2 \int\left(\hat{v}_{2}\right)^{2} \mu_{e}\left(\langle v\rangle, v_{2}\right) d v \bar{h}=\alpha \bar{h},
$$

$$
\begin{aligned}
\alpha & =-2 \int\left(\hat{v}_{2}\right)^{2} \mu_{e}\left(\langle v\rangle, v_{2}\right) d v=-2 \int \hat{v}_{2} \frac{\partial \mu}{\partial v_{2}} d v+2 \int \hat{v}_{2} \mu_{p} d v \\
& =2 \int \frac{\partial \hat{v}_{2}}{\partial v_{2}} \mu d v+4 C \int \sigma(\langle v\rangle) v_{2} \hat{v}_{2} d v=2 \int \frac{\partial^{2}\langle v\rangle}{\partial\left(v_{2}\right)^{2}} \mu d v+1>1
\end{aligned}
$$

since $\langle v\rangle$ is strictly convex in the variable $v_{2}$. Therefore

$$
\mathscr{L}(0) h=\lim _{\varepsilon \rightarrow 0} \mathscr{L}(\varepsilon)=-\partial_{x}^{2} h-h+\alpha \bar{h} .
$$

Now we decompose $h=\bar{h}+h^{\perp}$ where $\int h^{\perp} d x=0$. Then

$$
(\mathscr{L}(0) h, h)=\left(\left(-\partial_{x}^{2}-1\right) h^{\perp}, h^{\perp}\right)+(\alpha-1) \bar{h}^{2} \geq(\alpha-1) \bar{h}^{2} \geq 0
$$

since $\mathscr{M}(0)=-\partial_{x}^{2}-1$ takes functions of zero mean into functions of zero mean and is nonnegative on the periodic functions: $\mathscr{L}(0) \geq 0$. The operator $\mathscr{L}(0)$ has a two-dimensional kernel, spanned by $\cos x$ and $\sin x$ because if $h \in \operatorname{ker} \mathscr{L}(0)$, then the mean of $h$ vanishes so that $\partial_{x}^{2} h+h=0$.

We will show that for properly chosen $\sigma, \mathscr{L}(\varepsilon) \geq 0$ for $\varepsilon>0$ small. This implies that the equilibria with $\varepsilon>0$ are linearly stable. Moreover, we will show that 
$\mathscr{L}(\varepsilon)$ has only a one-dimensional kernel spanned by $\sin x$. This is also important in the nonlinear stability proof of [25]. We prove above properties of $\mathscr{L}(\varepsilon)$ in several steps. It suffices to prove $\mathscr{N}(\varepsilon)>\mathscr{N}(0)$ on $(\sin z)^{\perp}$.

Lemma 4.5. For any periodic function $\psi(x) \in L_{\text {per }}^{2}$, we have $\mathscr{P}_{\varepsilon}^{-}\left(v_{2} \psi\right)(x, v)=$ $\varepsilon \mathscr{P}_{1}^{-}\left(v_{2} \psi\right)(x, v / \varepsilon)$.

Proof. Denote $\tilde{D}_{\varepsilon}=v_{1} \partial_{x}-\varepsilon v_{2} B_{0} \partial_{v_{1}}+\varepsilon v_{1} B_{0} \partial_{v_{2}}$ without the "hats". Because $D_{\varepsilon}=$ $\frac{1}{\langle v\rangle} \tilde{D}_{\varepsilon}$, we have $\operatorname{ker} D_{\varepsilon}=\operatorname{ker} \tilde{D}_{\varepsilon}$. Furthermore, $\mathscr{P}_{\varepsilon}^{-}$can also be defined as the projection operator from $L_{\mu^{0}}^{2}$ to $\operatorname{ker} \tilde{D}_{\varepsilon}$. For any $h(x, v) \in \operatorname{ker} \tilde{D}_{1}$, we define $h_{\varepsilon}=$ $h(x, v / \varepsilon)$. Then

$$
\begin{aligned}
\tilde{D}_{\varepsilon} h_{\varepsilon} & =\left(v_{1} \partial_{x}-\varepsilon v_{2} B_{0} \partial_{v_{1}}+\varepsilon v_{1} B_{0} \partial_{v_{2}}\right) h_{\varepsilon} \\
& =\varepsilon\left(v_{1} \partial_{x} h-v_{2} B_{0} \partial_{v_{1}} h+v_{1} B_{0} \partial_{v_{2}} h\right)\left(x, \frac{v}{\varepsilon}\right) \\
& =\varepsilon \tilde{D}_{1} h\left(x, \frac{v}{\varepsilon}\right)=0
\end{aligned}
$$

so $h_{\varepsilon} \in \operatorname{ker} \tilde{D}_{\varepsilon}$ and each function in $\operatorname{ker} \tilde{D}_{\varepsilon}$ can be obtained in this way. By definition, for any $L_{\mu^{0}}^{2}-$ function $h \in \operatorname{ker} \tilde{D}_{1}$,

$$
\begin{aligned}
0 & =\int\left(v_{2} \psi-\mathscr{P}_{1}^{-}\left(v_{2} \psi\right)\right) h(x, v) d x d v \\
& =\frac{1}{\varepsilon^{3}} \int\left(v_{2} \psi-\varepsilon \mathscr{P}_{1}^{-}\left(v_{2} \psi\right)\left(x, \frac{v}{\varepsilon}\right)\right) h\left(x, \frac{v}{\varepsilon}\right) d x d v
\end{aligned}
$$

by the change of variable $v \rightarrow \frac{v}{\varepsilon}$. So $\left(v_{2} \psi-\mathscr{P}_{1}^{-}\left(v_{2} \psi\right)\left(x, \frac{v_{1}}{\varepsilon}\right), h_{\varepsilon}\right)=0$ which implies that $\mathscr{P}_{\varepsilon}^{-}\left(v_{2} \psi\right)(x, v)=\varepsilon \mathscr{P}_{1}^{-}\left(v_{2} \psi\right)\left(x, \frac{v}{\varepsilon}\right)$, since $h_{\varepsilon}$ can be an arbitrary $L_{\mu^{0}}^{2}-$ function in $\operatorname{ker} \tilde{D}_{\varepsilon}$.

Classification of Particles. In the following discussion we consider a general purely magnetic equilibrium $\left(E^{0}=0\right)$ with a $P$-periodic magnetic potential $\psi^{0}$, not depending on the above special form. We can adjust the starting point so that $\psi^{0}(0)=\psi^{0}(P)=\min \psi^{0}, \quad \psi^{0}\left(\frac{P}{2}\right)=\max \psi^{0}$, and $\psi^{0}$ is monotone in each half interval $\left[0, \frac{P}{2}\right]$ and $\left[\frac{P}{2}, P\right]$. The potential $\psi^{0}$ has a single minimum per period $P$.

Now we investigate the electron trajectories in the magnetic field $B_{0}=\partial_{x} \psi^{0}$. The ODE for any particle is

$$
\dot{X}=\hat{V}_{1}, \quad \dot{V}_{1}=-\hat{V}_{2} \partial_{x} \psi^{0}(X), \quad \dot{V}_{2}=\hat{V}_{1} \partial_{x} \psi^{0}(X) .
$$

The two invariants of (4.6) are the energy $e=\langle v\rangle$ and momentum $p=v_{2}-\psi^{0}(x)$. We classify the motion of the particles according to their energy and momentum $(e, p)$. We write

$$
d t=\frac{\langle v\rangle}{v_{1}} d x= \pm \frac{e d x}{\sqrt{e^{2}-1-\left(p+\psi^{0}(x)\right)^{2}}}
$$


Note that $e=\langle v\rangle, p=v_{2}-\psi^{0}(x)$ is equivalent to

$$
v_{1}= \pm \sqrt{e^{2}-1-\left(p+\psi^{0}(x)\right)^{2}}, \quad v_{2}=p+\psi^{0}(x) .
$$

$\left(A_{1}\right)$ (free particle) If $e^{2}>1+\max \left(p+\psi^{0}\right)^{2}$, the particle travels around the whole circle $[0, P]$ with the period

$$
T_{f}(e, p)=\int_{0}^{P} \frac{e d x}{\sqrt{e^{2}-1-\left(p+\psi^{0}(x)\right)^{2}}} .
$$

$\left(A_{2}\right)$ (trapped particle of type I) If $p \geq-\min \psi^{0}$ or $p \leq-\max \psi^{0}$, and $e^{2}<$ $1+\max \left(p+\psi^{0}\right)^{2}$, then $\left[p+\psi^{0}\right]^{2}$ has a single maximum at $P / 2$ and the particle is trapped in the interval $\left(a^{I}, b^{I}\right)$ with the half-period

$$
T_{t}^{I}(e, p)=\int_{a^{I}}^{b^{I}} \frac{e d x}{\sqrt{e^{2}-1-\left(p+\psi^{0}(x)\right)^{2}}},
$$

where $\left(a^{I}, b^{I}\right)$ is the interval such that $e^{2}-1-\left(p+\psi^{0}(x)\right)^{2} \geq 0$. On the other hand, if $-\max \psi^{0}<p<-\min \psi^{0}$, the function $\left(p+\psi^{0}(x)\right)^{2}$ has two maxima $M_{1} \leq M_{2}$ per period, which occur at $x=0, P / 2$.

$\left(A_{3}\right)$ (trapped particle of type II) If $-\max \psi^{0}<p<-\min \psi^{0}$ and $1+M_{1}<$ $e^{2}<1+M_{2}$, the particle is trapped in $\left(a^{I I}, b^{I I}\right)$ with half-period

$$
T_{t}^{I I}(e, p)=\int_{a^{I I}}^{b^{I I}} \frac{e d x}{\sqrt{e^{2}-1-\left(p+\psi^{0}(x)\right)^{2}}}
$$

where $\left(a^{I I}, b^{I I}\right)$ is the interval such that $e^{2}-1-\left(p+\psi^{0}(x)\right)^{2} \geq 0$.

$\left(A_{4}\right)$ (trapped particle of type III) If $-\max \psi^{0}<p<-\min \psi^{0}$ and $e^{2}<1+M_{1}$, the particle is trapped in one of the two intervals $\left(a_{1}^{I I I}, b_{1}^{I I I}\right)$ or $\left(a_{2}^{I I I}, b_{2}^{I I I}\right)$ where $0<a_{1}^{I I I}<b_{1}^{I I I}<\frac{P}{2}$ is such that $e^{2}-1-\left(p+\psi^{0}(x)\right)^{2}=0$ and $a_{2}^{I I I}=P-a_{1}^{I I I}, b_{2}^{I I I}=$ $P-b_{1}^{I I I}$. Its half-period is

$$
T_{t}^{I I I}(e, p)=\int_{a_{1}^{I I I}}^{b_{1}^{I I I}} \frac{e d x}{\sqrt{e^{2}-1-\left(p+\psi^{0}(x)\right)^{2}}} .
$$

$\left(A_{5}\right)$ (critical particle) If $e^{2}=1+\max \left(p+\psi^{0}\right)^{2}$, the particle motion is not periodic. It takes infinite time for the particle to approach the maximum point of $\left(p+\psi^{0}\right)^{2}$. The same situation occurs if the particle approaches the local maximum $e^{2}=1+M_{1}$.

The following lemma gives an explicit formula for $\mathscr{P}^{-}\left(\hat{v}_{2} \psi\right)$. 
Lemma 4.6. For any $\psi(x) \in L_{p e r}^{2}$, define the function $h_{\psi}(e, p)$ in the following way:

$$
h_{\psi}(e, p)=\frac{\int_{0}^{P} \frac{\left(p+\psi^{0}(x)\right) \psi(x) d x}{\sqrt{e^{2}-1-\left(p+\psi^{0}(x)\right)^{2}}}}{T_{f}(e, p)}
$$

for $(e, p)$ corresponding to a free particle, and

$$
h_{\psi}(e, p)=\frac{\int_{a}^{b} \frac{\left(p+\psi^{0}(x)\right) \psi(x) d x}{\sqrt{e^{2}-1-\left(p+\psi^{0}(x)\right)^{2}}}}{T_{t}(e, p)}
$$

for $(e, p)$ corresponding to a trapped particle where $(a, b)$ is the trapped interval of the particle. Then we have $\mathscr{P}^{-}\left(\hat{v}_{2} \psi\right)=h_{\psi}\left(\langle v\rangle, v_{2}-\psi^{0}(x)\right)$ in $L_{\left|\mu_{e}\right|}^{2}$.

Proof. By definition, $D\left(\mathscr{P}^{-}\left(\hat{v}_{2} \psi\right)\right)=0$ so $\mathscr{P}^{-}\left(\hat{v}_{2} \psi\right)$ is constant on each particle trajectory. For any compactly supported (in $v$ ) function $\xi \in \operatorname{ker} D$, we have (with $\varepsilon=1)$

$$
\begin{aligned}
0 & =\iiint\left(\hat{v}_{2} \psi-\mathscr{P}_{1}^{-}\left(\hat{v}_{2} \psi\right)\right) \xi(x, v) d x d v \\
& =\iiint_{I(e, p)}\left(\frac{p+\psi^{0}(x)}{e} \psi-\mathscr{P}_{1}^{-}\left(\hat{v}_{2} \psi\right)\right) \xi \frac{e}{\sqrt{e^{2}-1-\left(p+\psi^{0}(x)\right)^{2}}} d x d e d p
\end{aligned}
$$

where we changed variables $(x, v) \rightarrow(x, e, p)$ and used $I(e, p)$ to denote the $x$ interval with fixed $(e, p)$. We decompose the $(e, p)$ region into five parts $A_{1}, \cdots, A_{5}$ corresponding to the different type of particles defined above. We can ignore $A_{5}$ in the integral since it has zero measure. For $(e, p) \in A_{1} \cup A_{2} \cup A_{3}, I(e, p)$ is a single interval while $I(e, p)=\left(a_{1}^{I I I}, b_{1}^{I I I}\right) \cup\left(a_{2}^{I I I}, b_{2}^{I I I}\right)$ for $(e, p) \in A_{4}$. Since $\xi$ and 
$\mathscr{P}_{1}^{-}\left(\hat{v}_{2} \psi\right)$ are independent of $x$ on each component of $I(e, p)$, we have

$$
\begin{aligned}
0 & =\iint_{A_{1}} \int_{0}^{P}\left(\frac{p+\psi^{0}(x)}{e} \psi-\mathscr{P}_{1}^{-}\left(\hat{v}_{2} \psi\right)\right) \xi \frac{e}{\sqrt{e^{2}-1-\left(p+\psi^{0}(x)\right)^{2}}} d x d e d p \\
& +\iint_{A_{2}} \int_{a^{I}}^{b^{I}} \cdots d x d e d p+\iint_{A_{3}} \int_{a^{I I}}^{b^{I I}} \cdots d x d e d p+\iint_{A_{4}} \sum_{i=1}^{2} \int_{a_{i}^{I I I}}^{b_{i}^{I I I}} \cdots d x d e d p \\
& =\iint_{A_{1}} \xi\left(\int_{0}^{P} \frac{\left(p+\psi^{0}(x)\right) \psi(x) d x}{\sqrt{e^{2}-1-\left(p+\psi^{0}(x)\right)^{2}}}-\mathscr{P}_{1}^{-}\left(\hat{v}_{2} \psi\right) T_{f}(e, p)\right) d e d p \\
& +\iint_{A_{2}} \xi\left(\int_{a^{I}}^{b^{I}} \frac{\left(p+\psi_{0}(x)\right) \psi(x) d x}{\sqrt{e^{2}-1-\left(p+\psi^{0}(x)\right)^{2}}}-\mathscr{P}_{1}^{-}\left(\hat{v}_{2} \psi\right) T_{t}^{I}(e, p)\right) d e d p \\
& +\iint_{A_{3}} \xi\left(\int_{a^{I I}}^{b^{I I}} \frac{\left(p+\psi_{0}(x)\right) \psi(x) d x}{\sqrt{e^{2}-1-\left(p+\psi^{0}(x)\right)^{2}}}-\mathscr{P}_{1}^{-}\left(\hat{v}_{2} \psi\right) T_{t}^{I I}(e, p)\right) \operatorname{ded} p \\
& +\left.\iint_{A_{4}} \sum_{i=1,2} \xi\right|_{\left(a_{i}^{I I I}, b_{i}^{I I I}\right)} \int_{a_{i}^{I I I}}^{b_{i}^{I I}}\left(\frac{\left(p+\psi^{0}(x)\right) \psi(x) d x}{\sqrt{e^{2}-1-\left(p+\psi^{0}(x)\right)^{2}}}-\mathscr{P}_{1}^{-}\left(\hat{v}_{2} \psi\right) T_{t}^{I I I}(e, p)\right) \operatorname{ded} p .
\end{aligned}
$$

Since $\xi$ is arbitrary, each integrand must vanish. Thus $\mathscr{P}^{-}\left(\hat{v}_{2} \psi\right)=h_{\psi}(x, v)$ as claimed.

Using this lemma, we readily see by integration that $\mathscr{P}^{-}\left(\hat{v}_{2} \partial_{x} \psi^{0}\right)=0$. The following lemma shows that the converse is also true.

Lemma 4.7. For any $\psi \in C_{p e r}^{1}, \mathscr{P}^{-}\left(\hat{v}_{2} \psi\right)=0$ if and only if $\psi=c \partial_{x} \psi^{0}$.

Proof. Let $\mathscr{P}^{-}\left(\hat{v}_{2} \psi\right)=0$. First we choose $p>-\min \psi^{0}$ and $e$ slightly larger than $\min _{x} \sqrt{1+\left(p+\psi^{0}(x)\right)^{2}} \equiv m=\sqrt{1+\left(p+\psi^{0}(0)\right)^{2}}$. Looking at the graph of $\left(p+\psi^{0}\right)^{2}$, we see that the trapped particles are of type I and the trapped interval is a small interval $(-\varepsilon(e), \varepsilon(e))$ in the circle. As $e \searrow m$, the period $T_{t}^{I}(e, p)$ tends to a finite number because $\varepsilon(e)=O(\sqrt{e-m})$. Thus

$$
\frac{m^{2}-1}{m} \psi(0) \leftarrow \int_{-\varepsilon(e)}^{\varepsilon(e)} \frac{\left(p+\psi^{0}(x)\right) \psi(x) d x}{\sqrt{e^{2}-1-\left(p+\psi^{0}(x)\right)^{2}}} / T_{t}^{I}(e, p)=h_{\psi}(e, p)=\mathscr{P}^{-}\left(\hat{v}_{2} \psi\right)=0
$$

so that we must have $\psi(0)=\psi(P)=0$.

Secondly we choose $p<-\max \psi^{0}$ and $e$ slightly larger than $\min \sqrt{1+\left(p+\psi^{0}(x)\right)^{2}}=$ $\sqrt{1+\left(p+\psi^{0}\left(\frac{P}{2}\right)\right)^{2}}$. The trapped interval is a small interval centered at $\frac{P}{2}$. For the 
same reason as above, we have $\psi\left(\frac{P}{2}\right)=0$. Thus $\psi$ vanishes wherever $\partial_{x} \psi^{0}$ does (at $0, \frac{P}{2}$ and $P$ ).

Thirdly we choose $-\max \psi^{0}<p<-\min \psi^{0}$. Denote by $x_{1}^{p}, x_{2}^{p}$ the two roots of $p+\psi^{0}(x)=0$, where $0<x_{1}^{p}<\frac{P}{2}$ and $x_{2}^{p}=P-x_{1}^{p}$. Then the minimum of $\sqrt{1+\left(p+\psi^{0}(x)\right)^{2}}$ is obtained at both $x_{1}^{p}$ and $x_{2}^{p}$. We take $e$ to be slightly larger than $\sqrt{1+\left(p+\psi^{0}\left(x_{1}^{p}\right)\right)^{2}}$. The trapped particles are of type III. The two trapped intervals $I_{i}=\left(x_{i}^{p}-\varepsilon(e), x_{i}^{p}+\varepsilon(e)\right) \quad(i=1,2)$ are symmetric to each other with respect to $x=\frac{P}{2}$. We have $\varepsilon(e) \rightarrow 0$ and $T_{t}^{I I I}(e, p)$ tends to a finite number as $e \searrow \sqrt{1+\left(p+\psi^{0}\left(x_{1}^{p}\right)\right)^{2}} \equiv \ell$. From $0=\mathscr{P}^{-}\left(\hat{v}_{2} \psi\right)=h_{\psi}(e, p)$, we obtain

$$
\begin{aligned}
0 & =\int_{I_{i}} \frac{\left(p+\psi^{0}(x)\right) \psi(x) d x}{\sqrt{e^{2}-1-\left(p+\psi^{0}(x)\right)^{2}}} \\
& =\int_{I_{i}} \partial_{x}\left(\sqrt{e^{2}-1-\left(p+\psi^{0}(x)\right)^{2}}\right) \frac{\psi(x)}{\partial_{x} \psi^{0}} d x \\
& =-\int_{I_{i}} \sqrt{e^{2}-1-\left(p+\psi^{0}(x)\right)^{2}} \partial_{x}\left(\frac{\psi(x)}{\partial_{x} \psi^{0}}\right) d x
\end{aligned}
$$

Dividing by $\varepsilon(e)^{2}$ and taking the limit as $e \searrow \ell$, we obtain $\partial_{x}\left(\frac{\psi(x)}{\partial_{x} \psi^{0}}\right)=0$ at the point $x_{i}^{p}$, where $\frac{\psi(x)}{\partial_{x} \psi^{0}}$ is a well-defined function by the observation above. By changing $p$, the point $x_{i}^{p}$ can be any point in $(0, P)$. It follows that $\partial_{x}\left(\frac{\psi(x)}{\partial_{x} \psi^{0}}\right)$ vanishes everywhere and thus $\psi=c \partial_{x} \psi^{0}$ for some constant $c$.

Now we can construct some stable equilibria.

Example. We take a purely magnetic equilibrium with $\mu=\mu^{-}=\mu^{+}=C \sigma(\langle v\rangle)\left(1+p^{2}\right)$ and $\psi_{\varepsilon}^{0}(x)=\varepsilon \cos x$. We take $\sigma>0, \sigma^{\prime}<0$ on a finite interval $[0, a)$ while $\sigma \equiv 0$ on $[a, \infty)$. For $\varepsilon>0$ small, we will show that the operator $\mathscr{L}(\varepsilon)$ is nonnegative and $\operatorname{ker} \mathscr{L}(\varepsilon)$ is spanned by the single function $\sin x$. Thus this equilibrium is spectrally stable.

In order to prove this, we will show that $\mathscr{N}(\varepsilon)$ is strictly increasing. For any differentiable $\psi \in L_{\text {per }}^{2}$, define $g(\varepsilon)=(\mathscr{N}(\varepsilon) \psi, \psi)$. By Lemma 4.5 and the invariance of $\mu_{e}$ and of $\langle v\rangle$ under the flow,

$$
\begin{aligned}
g(\varepsilon) & =2 \iiint-\mu_{e}^{-}\left(\langle v\rangle, v_{2}-\varepsilon \cos x\right)\left[\mathscr{P}_{\varepsilon}^{-}\left(\hat{v}_{2} \psi\right)\right]^{2} d x d v \\
& =2 C \iiint-\sigma_{e}(\langle v\rangle)\left(1+\left(v_{2}-\varepsilon \cos x\right)^{2}\right) \frac{1}{\langle v\rangle^{2}} \varepsilon^{2}\left[\mathscr{P}_{1}^{-}\left(v_{2} \psi\right)\right]^{2}\left(x, \frac{v}{\varepsilon}\right) d x d v \\
& =-2 C \iiint \varepsilon^{6} \sigma_{e}(\langle\varepsilon v\rangle) \frac{1}{\langle\varepsilon v\rangle^{2}}\left(\frac{1}{\varepsilon^{2}}+\left(v_{2}-\cos x\right)^{2}\right)\left[\mathscr{P}_{1}^{-}\left(v_{2} \psi\right)\right]^{2}(x, v) d x d v
\end{aligned}
$$


by changing scale in $v$. Denoting $H(e)=-\sigma^{\prime}(e) / e^{2}$, we have

$$
\begin{aligned}
g^{\prime}(\varepsilon) & =2 C \iiint \varepsilon^{3}\left(4 H(\langle\varepsilon v\rangle)+H^{\prime}(\langle\varepsilon v\rangle) \frac{\varepsilon|v|^{2}}{\langle\varepsilon v\rangle}\right)\left[\mathscr{P}_{1}^{-}\left(v_{2} \psi\right)\right]^{2} d x d v \\
& +2 C \iiint \varepsilon^{5}\left(6 H(\langle\varepsilon v\rangle)+H^{\prime}(\langle\varepsilon v\rangle) \frac{\varepsilon|v|^{2}}{\langle\varepsilon v\rangle}\right)\left(v_{2}-\cos x\right)^{2}\left[\mathscr{P}_{1}^{-}\left(v_{2} \psi\right)\right]^{2} d x d v .
\end{aligned}
$$

Since $\sigma$ has compact support, if $\varepsilon>0$ is small enough, we have

$$
4 H(\langle\varepsilon v\rangle)+H^{\prime}(\langle\varepsilon v\rangle) \frac{\varepsilon|v|^{2}}{\langle\varepsilon v\rangle}=4 H(1)+O(\varepsilon)>3 H(1)=-3 \sigma^{\prime}(1)>0 .
$$

By Lemma 4.7, if $\psi$ is not a multiple of $\sin x$, then $\mathscr{P}_{1}^{-}\left(v_{2} \psi\right) \neq 0$ and thus $g^{\prime}(\varepsilon)>$ 0 for small $\varepsilon$. This implies that $g(\varepsilon)>g(0)=(\mathscr{N}(0) \psi, \psi)$. Therefore $(\mathscr{L}(\varepsilon) \psi, \psi)>$ $(\mathscr{L}(0) \psi, \psi)$ for $\psi \neq c \sin x$. Recalling that $\sin x \in \operatorname{ker} \mathscr{L}(\varepsilon)$ and $\mathscr{L}(0) \geq 0$, we conclude that $\mathscr{L}(\varepsilon) \geq 0$ and that $\operatorname{ker} \mathscr{L}(\varepsilon)$ is spanned by $\sin x$ for small $\varepsilon$.

Remark. Combining this example with Theorem 4.4, we have constructed a periodic purely magnetic equilibrium that is stable under perturbations of minimal period and unstable under perturbations of double the period. It is worthwhile to make a comparison with the homogeneous case. By Theorem 4.1, for any homogeneous equilibrium, the perturbations of longer wave lengths are always more dangerous for stability. This example indicates that the same phenomenon is valid for an inhomogeneous equilibrium. We believe in general that perturbations of longer wave length are more likely to induce instability.

Remark. If $4\left|\sigma^{\prime}\right|>\sigma^{\prime \prime}$ as $e \rightarrow \infty$, for example if $\sigma(e)=(1+e)^{-2}$, then $g^{\prime}(\varepsilon) \geq 0$, hence $(\mathscr{L}(\varepsilon) \psi, \psi) \geq 0$ for small $\varepsilon$. This follows immediately without using Lemmas 15 or 16 . In some cases we can simply choose $\sigma$ so that $4 H(0)+H^{\prime 2} /\langle s\rangle^{2} \geq 0$.

\section{Appendix}

In this appendix, we construct some equilibria of the type studied in this paper. For the $1 \frac{1}{2} \mathrm{D}$ case, we prove that there exist infinitely many periodic equilibria. Then for the 3D case, we prove that for properly chosen external potentials, there exist equilibria representing a compactly confined plasma.

\section{Existence of periodic equilibria in $1 \frac{1}{2}$ dimensions}

We consider a plasma with two species. The equilibrium is

$$
f^{0, \pm}=\mu^{ \pm}\left(e^{ \pm}, p^{ \pm}\right)=\mu^{ \pm}\left(\langle v\rangle \pm \phi^{0}(x), v_{2} \pm \psi^{0}(x)\right) .
$$

For simplicity, we choose $\mu^{+}=\mu^{-}=\mu$. Given $\mu$, the equilibrium potentials $\left(\phi^{0}, \psi^{0}\right)$ should satisfy the ODE system

$$
\left\{\begin{array}{c}
\partial_{x}^{2} \phi=-\rho=-\int\left(\mu\left(\langle v\rangle+\phi, v_{2}+\psi\right)-\mu\left(\langle v\rangle-\phi, v_{2}-\psi\right)\right) d v \\
\partial_{x}^{2} \psi=-j_{2}=-\int \hat{v}_{2}\left(\mu\left(\langle v\rangle+\phi, v_{2}+\psi\right)-\mu\left(\langle v\rangle-\phi, v_{2}-\psi\right)\right) d v
\end{array}\right.
$$


We claim that for many choices of $\mu$ there exist many periodic solutions of this system. In order to show this, we first write the system in Hamiltonian form. Let $\partial_{x}$ be written as a dot and let

$$
q_{1}=\psi, q_{2}=\phi, r_{1}=-\dot{\psi}, r_{2}=\dot{\phi}, q=\left(q_{1}, q_{2}\right), r=\left(r_{1}, r_{2}\right) .
$$

Let

$$
\begin{aligned}
V(q) & =\int_{0}^{q_{2}} \int\left(\mu\left(\langle v\rangle+\lambda, v_{2}+q_{1}\right)-\mu\left(\langle v\rangle-\lambda, v_{2}-q_{1}\right)\right) d v d \lambda \\
& +\int_{0}^{q_{1}} \int \hat{v}_{2}\left(\mu\left(\langle v\rangle, v_{2}-\lambda\right)-\mu\left(\langle v\rangle, v_{2}+\lambda\right)\right) d v d \lambda
\end{aligned}
$$

Note that $\frac{\partial V}{\partial q_{2}}=\rho$ and

$$
\begin{aligned}
\frac{\partial V}{\partial q_{1}} & =\int_{0}^{q_{2}} \int\left(\mu_{p}\left(\langle v\rangle+\lambda, v_{2}+q_{1}\right)+\mu_{p}\left(\langle v\rangle-\lambda, v_{2}-q_{1}\right)\right) d v d \lambda \\
& +\int \hat{v}_{2}\left(\mu\left(\langle v\rangle, v_{2}-q_{1}\right)-\mu\left(\langle v\rangle, v_{2}+q_{1}\right)\right) d v \\
& =\int_{0}^{q_{2}} \int\left\{\frac{\partial}{\partial v_{2}}-\hat{v}_{2} \frac{\partial}{\partial \lambda}\right\}\left\{\mu\left(\langle v\rangle+\lambda, v_{2}+q_{1}\right)\right\} d v d \lambda \\
& +\int_{0}^{q_{2}} \int\left\{\frac{\partial}{\partial v_{2}}+\hat{v}_{2} \frac{\partial}{\partial \lambda}\right\}\left\{\mu\left(\langle v\rangle-\lambda, v_{2}-q_{1}\right)\right\} d v d \lambda \\
& +\int \hat{v}_{2}\left(\mu\left(\langle v\rangle, v_{2}-q_{1}\right)-\mu\left(\langle v\rangle, v_{2}+q_{1}\right)\right) d v \\
& =-\int \hat{v}_{2}\left(\mu\left(\langle v\rangle+\phi, v_{2}+\psi\right)-\mu\left(\langle v\rangle-\phi, v_{2}-\psi\right)\right) d v=-j_{2} .
\end{aligned}
$$

Let $S$ be the diagonal matrix with entries $(-1,1)$ and let

$$
H(q, r)=-\frac{1}{2} r_{1}^{2}+\frac{1}{2} r_{2}^{2}+V\left(q_{1}, q_{2}\right)=(S r) \cdot r+V(q) .
$$

Then our system takes the Hamiltonian form

$$
\dot{q}=S r=\frac{\partial H}{\partial r}, \quad-\dot{r}=V^{\prime}(q)=\frac{\partial H}{\partial q} .
$$

We shall apply a theorem of Hofer and Toland [18].

Theorem 4.8. Let $V$ be a $C^{3}$ function $: \mathbb{R}^{2} \rightarrow \mathbb{R}$. Let $\mathscr{C}$ be the closure of a component of $\left\{q \in \mathbb{R}^{2} \mid V(q)>0\right\}$. Assume that

(i) $\mathscr{C}$ is compact and convex;

(ii) $V^{\prime}(q) \neq 0$ for $q \in \partial \mathscr{C}$;

(iii) if $q \in \partial \mathscr{C}$ and $\left(S V^{\prime}(q), V^{\prime}(q)\right)=0$, then $\left(V^{\prime \prime}(q) S V^{\prime}(q), S V^{\prime}(q)\right)<0$.

Then there exists a periodic orbit $\{(q(t), r(t)) \mid t \in \mathbb{R}\}$ with some positive minimal period $T$ with $q$ even and $r$ odd and with the properties

$$
r(0)=r(T / 2)=0, q(0) \in \partial \mathscr{C}, q(T / 2) \in \partial \mathscr{C}, q((0, T / 2)) \subset \operatorname{int}(\mathscr{C}) .
$$


Moreover, $q(t)$ is monotone in $[0, T / 2]$ in the sense that $q\left(t_{2}\right)-q\left(t_{1}\right)$ is in the positive cone $C_{S}^{+}=\left\{q \mid q_{1}>0,(S q, q)<0\right\}$ for $T / 2>t_{2}>t_{1}>0$.

Corollary. If $V$ is a $C^{3}$ function which has a maximum point $q^{o}$ where its Hessian $V^{\prime \prime}<0$, then there exist a family of periodic solutions in a neighborhood of $q^{o}$.

Proof. We have $V^{\prime}\left(q^{o}\right)=0, V^{\prime \prime}\left(q^{o}\right)<0$. Then of course all the level sets near $q^{o}$ are convex and bounded. On the level sets near $q^{o}$, we have $V^{\prime} \neq 0$ and $V^{\prime \prime}<0$. Therefore all the hypotheses of the theorem are satisfied.

In order to explicitly exhibit some choices of $\mu$ that satisfy these conditions, we choose $q^{o}$ to be the origin. Then obviously the first derivatives of $V$ are zero at $q^{o}$. First we choose $\mu(e, p)$ to be a positive even function of $p$ such that $p \mu_{p}(e, p)>0$ for $p \neq 0$ and $\mu_{e}<0$. This is easily arranged, for instance, if $\mu(e, p)=\alpha(e) \beta(p)$ for appropriate $\alpha$ and $\beta$. Now

$$
\frac{\partial^{2} V}{\partial q_{1} \partial q_{2}}=\frac{\partial \rho}{\partial q_{1}}=\int\left(\mu_{p}\left(\langle v\rangle+q_{2}, v_{2}+q_{1}\right)+\mu_{p}\left(\langle v\rangle-q_{2}, v_{2}-q_{1}\right)\right) d v
$$

vanishes at the origin $q=0$. Furthermore, both

$$
\frac{\partial^{2} V}{\partial q_{2}^{2}}=\int\left(\mu_{e}\left(\langle v\rangle+q_{2}, v_{2}+q_{1}\right)+\mu_{e}\left(\langle v\rangle-q_{2}, v_{2}-q_{1}\right)\right) d v
$$

and

$$
\frac{\partial^{2} V}{\partial q_{1}^{2}}=-\int \hat{v}_{2}\left(\mu_{p}\left(\langle v\rangle+q_{2}, v_{2}+q_{1}\right)+\mu_{p}\left(\langle v\rangle-q_{2}, v_{2}-q_{1}\right)\right) d v
$$

are negative at the origin. Thus the corollary is applicable. This construction provides only a purely magnetic equilibrium since $(0, \psi)$ is an invariant plane for the ODE system (4.1), by the evenness of $\mu$ in $p$.

To get a truly electromagnetic equilibrium with $\mathbf{E}^{0} \neq \mathbf{0}$, we modify the above even example to break the evenness in $p$. If we add a small odd part to the even example above, then $V$ still has a strict maximum nearby and so the corollary is still applicable. In this case, $(0, \psi)$ is no longer an invariant plane for (4.1) and we get equilibrium with $\mathbf{E}^{0} \neq \mathbf{0}$. Thus we can construct infinitely many periodic electromagnetic equilibria $\left(\psi^{0}, \phi^{0}\right)$. Moreover, by Theorem 4.8 , we easily derive the following properties of the equilibrium. First, $\psi^{0}, \phi^{0}$ are even functions in $[0, T]$. Second, $\psi^{0}$ is increasing in $\left[0, \frac{T}{2}\right]$ and decreasing in $\left[\frac{T}{2}, T\right]$.

Remark. By a similar process, solitary wave and kink type solutions can also be constructed for (4.1) by using the corresponding theorems in [18] for homoclinic and heteroclinic orbits. These types of solutions are also constructed in [8] by using more complicated dynamical system arguments. However, a periodic equilibrium was not constructed in [8]. 


\section{Existence of 3D confined symmetric equilibria}

To accomplish this, we need an external field. Indeed, for the three-dimensional problem there can be no equilibria of finite energy in the absence of an external field. In fact, it is proven in [16] that every finite-energy solution of RVM without an external field in 3D must satisfy the estimate

$$
\int_{0}^{\infty} \int_{|x| \leq R}\left\{\frac{1}{2}|\mathbf{E}|^{2}+\frac{1}{2}|\mathbf{B}|^{2}+\int_{\mathbb{R}^{3}}\langle v\rangle f d v\right\} d x d t<\infty
$$

or all $R<\infty$. It is clear that this could not be satisfied by an equilibrium. The estimate (4.4) is also true for a multi-species plasma without an external field. Thus, to get any 3D plasma equilibrium, it is necessary to add an external electromagnetic field. For the case of MHD, the necessity of external fields for the existence of equilibria in the whole space is well known in the physics literature (see [6]).

Now we construct one type of confined plasma with support in a torus. Our method of proof is a modification of the standard sub-sup argument, as in [1] and [26], but there are some major differences. In [1] and [10] steady states are constructed in a bounded domain away from the $z$-axis with the perfectly conducting boundary condition. Here we consider the confinement problem in free space with external fields, a more practical problem in physics ([3]). One difficulty in our proof is that the operator $-\partial_{z z}-\partial_{r r}-\frac{1}{r} \partial_{r}+\frac{1}{r^{2}}$ that appears in the steady state equation appears to be singular at $z$-axis. That is why the existence results of [1] and [10] could only be proved under the restriction that the domain is away from $z$-axis. However, observation (3.4) allows this restriction to be removed so that equilibria with continuous fields in the whole domain including the $z$-axis can be constructed.

We consider an equilibrium $\left(f^{0}, \phi^{0}, A_{\theta}^{0}\right)$ of the form $f^{0}=F(e) G\left(p_{\theta}\right)$, where

$$
e=\sqrt{1+|v|^{2}}-\phi^{0}(r, z)-\phi^{e x t}(r, z), \quad p_{\theta}=r\left(v_{\theta}-A_{\theta}^{0}(r, z)\right)
$$

and $\left(\phi^{0}, A_{\theta}^{0}\right)$ satisfies the elliptic system

$$
\left\{\begin{array}{c}
\partial_{z z} \phi^{0}+\partial_{r r} \phi^{0}+\frac{1}{r} \partial_{r} \phi^{0}=\rho=\int F(e) G\left(p_{\theta}\right) d v \\
\partial_{z z} A_{\theta}^{0}+\partial_{r r} A_{\theta}^{0}+\frac{1}{r} \partial_{r} A_{\theta}^{0}-\frac{1}{r^{2}} A_{\theta}^{0}=j_{\theta}=\int \hat{v}_{\theta} F(e) G\left(p_{\theta}\right) d v
\end{array}\right.
$$

Denoting $U=\phi^{0}(r, z)+\phi^{e x t}(r, z),(4.5)$ becomes

$\left\{\begin{array}{c}\Delta U=n_{1}(r, z)+\rho\left(U, A_{\theta}^{0}\right)=n_{1}(r, z)+\int F\left(\sqrt{1+|v|^{2}}-U\right) G\left(r\left(v_{\theta}-A_{\theta}^{0}\right)\right) d v \\ \Delta_{\text {mag }} A_{\theta}^{0}=\Delta A_{\theta}^{0}-\frac{1}{r^{2}} A_{\theta}^{0}=j_{\theta}\left(U, A_{\theta}^{0}\right)=\int \hat{v}_{\theta} F\left(\sqrt{1+|v|^{2}}-U\right) G\left(r\left(v_{\theta}-A_{\theta}^{0}\right)\right) d v\end{array}\right.$

where $n_{1}(r, z)=\Delta \phi^{\text {ext }}(r, z)$.

We impose the following assumptions:

i) $F, G \in C^{1}(\mathbb{R}), F \geq 0, G \geq 0, G$ has bounded $C^{1}$ norm.

ii) $F(e) e \sqrt{e^{2}-1},\left|F^{\prime}(e)\right| e \sqrt{e^{2}-1} \leq m(e)$ for some $m \in L^{1}((1, \infty))$. 
iii) $F(s)=0$ if $s>e_{0}$ for some $e_{0}<1$.

iv) $G(s)=0$ if $|s|<\delta_{0}$ for some $\delta_{0}>0$.

v) $n_{1} \in C_{c}^{1}\left(\mathbb{R}^{3}\right)$ and $n_{1} \leq 0$ is compactly supported.

It is trivial to construct functions $F$ and $G$ satisfying above assumptions.

Theorem 4.9. Under Assumptions $i$ )-v), there exists an equilibrium with $\left(\phi^{0}, A_{\theta}^{0}\right) \in$ $C^{2}\left(\mathbb{R}^{3}\right)$ such that the equilibrium density $\rho^{0}$ and the current $j_{\theta}^{0}$ are supported in a torus; specifically, they are supported in a region $\left\{(r, z)\left|0<C_{1} \leq r \leq C_{2},\right| z \mid \leq C_{3}\right\}$.

Proof. We recall from (3.4) that for a function $f(r, z) \in H^{2 \dagger}$ we have

$$
(-\Delta)_{m a g} f:=\left(-\partial_{z z}-\partial_{r r}-\frac{1}{r} \partial_{r}+\frac{1}{r^{2}}\right) f=e^{-i \theta}(-\Delta)\left(e^{i \theta} f\right) .
$$

This shows how the singular term $1 / r^{2}$ is artificially introduced by the coordinate change. On any cylindrically symmetric, bounded domain $\Omega$, we can define $\left(-\Delta_{\Omega}\right)_{\text {mag }}^{-1}: L_{S}^{2}(\Omega) \rightarrow H^{2 \dagger}(\Omega)$ by

$$
\left(-\Delta_{\Omega}\right)_{m a g}^{-1}=e^{-i \theta}\left(-\Delta_{\Omega}\right)^{-1}\left(e^{i \theta}\right)
$$

where $\left(-\Delta_{\Omega}\right)^{-1}$ is defined by the Dirichlet boundary condition. It is obvious that $\left(-\Delta_{\Omega}\right)_{m a g}^{-1}$ is a compact operator. Moreover, we still have the maximum principle for $\left(-\Delta_{\Omega}\right)_{m a g}$. So we can modify the theorem of McKenna-Walter (see also Theorem 4.1 in [1]) to directly solve our system (4.6) in a bounded domain by the sub-sup method. There is very little change in the standard proof so we skip the details. We use the sub-sup method to solve problem (4.6) in $B_{R}=\{x|| x \mid \leq R\}$, with the boundary condition $U=A_{\theta}^{0}=0$ on $\partial B_{R}$.

By a straightforward calculation (see [1]), Assumption ii) implies that $\left|j_{\theta}\right| \leq$ $\rho \leq C^{\prime}$ for some constant $C^{\prime}$. Define $A_{2}^{R}=\left(-\Delta_{B_{R}}\right)_{\text {mag }}^{-1}\left(C^{\prime}\right), A_{1}^{R}=\left(-\Delta_{B_{R}}\right)_{\text {mag }}^{-1}\left(-C^{\prime}\right)$, $U_{2}^{R}=\left(-\Delta_{B_{R}}\right)^{-1}\left(-n_{1}\right)$ and $U_{1}^{R}=\left(-\Delta_{B_{R}}\right)^{-1}\left(-n_{1}-C^{\prime}\right)$. Then for all $\beta \in\left[U_{1}^{R}, U_{2}^{R}\right]$ and $\alpha \in\left[A_{1}^{R}, A_{2}^{R}\right]$ we have $-\Delta U_{2}^{R}=-n_{1}(r, z) \geq-n_{1}(r, z)-\rho(\beta, \alpha)$ and $-\Delta U_{1}^{R}=$ $-n_{1}-C^{\prime} \leq-n_{1}(r, z)-\rho(\beta, \alpha)$. We also have $(-\Delta)_{m a g} A_{2}^{R}=C^{\prime} \geq j_{\theta}(\beta, \alpha)$ and $(-\Delta)_{\text {mag }} A_{1}^{R}=-C^{\prime} \leq j_{\theta}(\beta, \alpha)$. So $\left(U_{1}^{R}, A_{1}^{R}\right)$ and $\left(U_{2}^{R}, A_{2}^{R}\right)$ are sub and sup solutions to (4.6) on $B_{R}$. By the modified form of the McKenna-Walter Theorem mentioned above, there exists a $C^{2+\delta}$ solution $\left(U^{R}, A_{\theta}^{R}\right)$ of (4.6) on $B_{R}$ vanishing on $\partial B_{R}$ and satisfying $U_{1}^{R} \leq U^{R} \leq U_{2}^{R}$ and $A_{1}^{R} \leq A_{\theta}^{R} \leq A_{2}^{R}$ for some $0<\delta<1$. We denote $\rho^{R}=\rho\left(U^{R}, A_{\theta}^{R}\right)$ and $j_{\theta}^{R}=j_{\theta}\left(U^{R}, A_{\theta}^{R}\right)$.

If we also denote $U_{2}^{\infty}=-\int \frac{n_{1}(y)}{|x-y|} d y$, then of course $U_{2}^{R} \nearrow U_{2}^{\infty}$ as $R \rightarrow \infty$. Since $U_{2}^{\infty}(x) \rightarrow 0$ as $|x| \rightarrow \infty$, there exists $R_{0}>0$ such that $1-U_{2}^{\infty}(x) \geq e_{0}$ for $|x| \geq R_{0}$. So if $R \geq R_{0}$, we have

$$
\sqrt{1+|v|^{2}}-U^{R}(x) \geq \sqrt{1+|v|^{2}}-U_{2}^{R}(x) \geq \sqrt{1+|v|^{2}}-U_{2}^{\infty}(x) \geq e_{0}
$$

for $|x| \geq R_{0}$. By Assumption iii) it follows that $\rho^{R}$ and $j_{\theta}^{R}$ have their supports in the ball $B_{R_{0}}$ for any $R \geq R_{0}$. 
Furthermore by Assumptions i) and ii), $\rho^{R}$ and $j_{\theta}^{R}$ are bounded in $C^{1}\left(\mathbb{R}^{3}\right)$ uniformly as $R \rightarrow \infty$. Thus there exist cylindrically symmetric $\rho^{\infty}$ and $j_{\theta}^{\infty}$ in $C^{\delta}\left(\mathbb{R}^{3}\right)$ with supports in $B_{R_{0}}$ such that $\left(\rho^{R}, j_{\theta}^{R}\right) \rightarrow\left(\rho^{\infty}, j_{\theta}^{\infty}\right)$ in $C^{2}\left(\mathbb{R}^{3}\right)$ as $R \rightarrow \infty$. Hence there also exists a pair $\left(U^{\infty}, A_{\theta}^{\infty}\right) \in C^{2}\left(B_{R_{0}}\right)$ such that $\left(U^{R}, A_{\theta}^{R}\right) \rightarrow\left(U^{\infty}, A_{\theta}^{\infty}\right)$ in $C^{\delta}\left(B_{R_{0}}\right)$ as $R \rightarrow \infty$. Thus $\left(U^{\infty}, A_{\theta}^{\infty}\right)$ clearly solves (4.6) in $B_{R_{0}}$ with $\rho^{\infty}=$ $\rho\left(U^{\infty}, A_{\theta}^{\infty}\right)$ and $j_{\theta}^{\infty}=j_{\theta}\left(U^{\infty}, A_{\theta}^{\infty}\right)$. We extend $\left(U^{\infty}, A_{\theta}^{\infty}\right)$ outside the fixed ball $B_{R_{0}}$ by setting $U^{\infty}(x)=U_{2}^{\infty}(x)$ and $A_{\theta}^{\infty}=0$. It satisfies (4.6) in $\left(B_{R_{0}}\right)^{c}$. Then $\left(U^{\infty}, A_{\theta}^{\infty}\right)$ solves (4.6) in the whole space $\mathbb{R}^{3}$.

That $\operatorname{supp}\left(\rho^{\infty}\right) \subset\left\{(r, z) \mid r \leq C_{2}, z \leq C_{3}\right\}$ is implied by the compact support of $\rho^{\infty}$. To show that $\operatorname{supp}\left(\rho^{\infty}\right) \subset\left\{(r, z) \mid r \geq C_{1}\right\}$, we use Assumption iv). For very small $r$, in order for $G\left(r\left(v_{\theta}-A_{\theta}^{\infty}\right)\right)$ to be non-zero, we must take $\left|v_{\theta}\right|$ to be very

big, by Assumption iv). Thus if $r$ is small enough, we have $\sqrt{1+|v|^{2}}-U^{\infty}>e_{0}$ which implies that $\rho^{\infty}=0$. So $\operatorname{supp}\left(\rho^{\infty}\right) \subset\left\{(r, z) \mid r \geq C_{1}\right\}$ for some $C_{1}>0$. Let $\rho^{0}=\rho^{\infty}$ and $j_{\theta}^{0}=j_{\theta}^{\infty}$.

Acknowledgment. We thank Helmut Hofer for pointing out reference [18] and Fanghua Lin for showing us the important identity (3.4). We also thank Yan Guo for many helpful comments. The work was supported in part by the NSF under Grants DMS-0405066 and DMS-0505460.

\section{Bibliography}

[1] Batt, Jürgen and Fabian, Karl, Stationary solutions of the relativistic Vlasov-Maxwell system of plasma physics. Chinese Ann. Math. Ser. B 14 (1993), no. 3, 253-278.

[2] Binney, James and Tremaine, Scott, Galactic dynamics, Princeton University Press, 1987.

[3] Davidson, Ronald C., Physics of Nonneutral Plasmas, Addison-Wesley 1990.

[4] Davidson, Ronald C. and Hong, Qin, Physics of intense charged particle beams in high energy accelerators, World Scientific, 2001.

[5] DiPerna, Ronald J., Lions, Pierre-Louis, Meyer, Yves, $L^{p}$-regularity of velocity averages. Ann. Inst. H. Poincaré, Anal. Non Linéaire 8 (1991), no. 3-4, 271-287.

[6] Friedberg, J. P., Ideal Magnetohydrodynamics, Plenum Press, 1987.

[7] Goedbloed, Hans and Poedts, Stefan, Principles of Magnetohydrodynamics : With Applications to Laboratory and Astrophysical Plasmas, Cambridge University Press, 2004.

[8] Guo, Yan and Grotta Ragazzo, Clodoaldo On steady states in a collisionless plasma. Comm. Pure Appl. Math. 49 (1996), no. 11, 1145-1174.

[9] Guo, Yan, Stable magnetic equilibria in collisionless plasma, Comm. Pure Appl. Math., Vol L, 0891-0933 (1997).

[10] Guo, Yan, Stable magnetic equilibria in a symmetric plasma, Commun. Math. Phys., 200, 211247 (1999).

[11] Guo, Yan and Strauss, Walter, Instability of periodic BGK equilibria, Comm. Pure Appl. Math. Vol XLVIII, 861-894 (1995).

[12] Guo, Yan and Strauss, Walter, Unstable BGK solitary waves and collisionless shocks, Commun. Math. Phys. 195, 249-265 (1998).

[13] Guo, Yan and Strauss, Walter, Unstable oscillatory-tail waves in collisionless plasmas, SIAM J. Math. Anal., 30, no. 5, 1076-1114 (1999). 
[14] Guo, Yan and Strauss, Walter, Unstable relativistic BGK waves, Comp. and Appl. Math., 18, no. 1, 87-122 (1999)

[15] Guo, Yan and Strauss, Walter, Magnetically created instability in a collisionless plasma, J. Math. Pures. Appl., 79, no. 10, 975-1009 (2000).

[16] Glassey, Robert and Strauss, Walter, Remarks on collisionless plasmas. Fluids and plasmas: geometry and dynamics (Boulder, Colo., 1983), 269-279, Contemp. Math., 28, Amer. Math. Soc., Providence, RI, 1984.

[17] Glassey, Robert and Schaeffer, Jack, On the "one and one-half dimensional" relativistic VlasovMaxwell system. Math. Methods Appl. Sci. 13 (1990), no. 2, 169-179.

[18] Hofer, Helmut and Toland, John, Homoclinic, heteroclinic, and periodic orbits for a class of indefinite Hamiltonian systems. Math. Ann. 268 (1984), no. 3, 387-403.

[19] Lavel, G., Mercier, C. and Pellat, R.M., Necessity of the energy principle for magnetostatic stability, Nuclear Fusion 5, 156-158 (1965).

[20] Lin, Zhiwu, Instability of periodic BGK waves, Math. Res. Letts., 8, 521-534(2001).

[21] Lin, Zhiwu, Some stability and instability criteria for ideal plane flows, Comm. Math. Phys., 246, no. 1, 87-112(2004).

[22] Lin, Zhiwu, Nonlinear instability of periodic waves for Vlasov-Poisson system, Comm. Pure. Appl. Math. Vol LVIII, 505-528 (2005).

[23] Lin, Zhiwu, Nonlinear instability of ideal plane flows, Int. Math. Res. Not., 41, 2147-2178 (2004).

[24] Lin, Zhiwu, Ph. D. thesis, Brown University, (2003).

[25] Lin, Zhiwu and Strauss, Walter, Nonlinear stability and instability of relativistic VlasovMaxwell systems, to appear in Comm. Pure. Appl. Math.

[26] McKenna, Patrick and Walter, Wolfgang, On the Dirichlet problem for elliptic systems. Appl. Anal. 21 (1986), no. 3, 207-224.

[27] Nicholson. D. R., Introduction to plasma theory, Wiley, 1983.

[28] Parks, George, Physics of space plasmas, Second Edition, Westview Press, 2004.

[29] Taylor, J.B., Plasma Containment and Stability Theory, Proceedings of the Royal Society of London. Series A, Mathematical and Physical Sciences, Vol. 304, No. 1478. (1968), pp. 335360.

[30] Trivelpiece, A. W. and Krall, N. A., Principles of plasma physics, McGraw-Hill, 1973.

Received Month 200X. 